\title{
Argon Permeability of Graphite Fuel Elements for the High-Temperature Gas-Cooled Reactor
}
A. J. Caputo
D. R. Johnson
C. K. Bayne

\section{OAK RIDGE NATIONAL LABORATORY}




\section{DISCLAIMER}

This report was prepared as an account of work sponsored by an agency of the United States Government. Neither the United States Government nor any agency Thereof, nor any of their employees, makes any warranty, express or implied, or assumes any legal liability or responsibility for the accuracy, completeness, or usefulness of any information, apparatus, product, or process disclosed, or represents that its use would not infringe privately owned rights. Reference herein to any specific commercial product, process, or service by trade name, trademark, manufacturer, or otherwise does not necessarily constitute or imply its endorsement, recommendation, or favoring by the United States Government or any agency thereof. The views and opinions of authors expressed herein do not necessarily state or reflect those of the United States Government or any agency thereof. 


\section{DISCLAIMER}

Portions of this document may be illegible in electronic image products. Images are produced from the best available original document. 


\section{Printed in the United States of America. Available from National Technical Information Service \\ U.S. Department of Commerce 5285 Port Royal Road, Springfield, Virginia 22161 \\ Price Printed Copy $\$ 4,50$; Microfiche $\$ 3.00$}

This report was prepared as an account of work sponsored by the United States Government. Neither the United States nor the Energy Research and Development Administration/United States Nuclear Regulatory Commission, nor any of their employees, nor any of their contractors, subcontractors, or their employees, makes any warranty, express or implied, or assumes any legal liability or responsibility for the accuracy, completeness or usefulness of any information, apparatus, product or process disclosed, or represents that its use would not infringe privately owned rights. 
ORNL/TM-5816

Distribution

Category UC-77

Contract No. W-7405-eng- 26

METALS AND CERAMICS DIVISION

THORIUM UTILIZATION PROGRAM (189a OHO45)

Fabrication Development - Task $\mathbf{3 0 0}$

ARGON PERMEABILITY OF GRAPHITE FUEL ELEMENTS FOR THE HIGH-TEMPERATURE GAS-COOLED REACTOR

A. J. Caputo, D. R. Johnson, C. K. Bayne

Date Published: May 1977

\footnotetext{
OAK RIDGE NATIONAL LABORATORY

Oak Ridge, Tennessee 37830

operated by

UNION CARBIDE CORPORATION

for the

ENERGY RESEARCH AND DEVELOPMENT ADMINISTRATION
} 
THIS PAGE

\section{WAS INTENTIONALLY \\ LEFT BLANK}




\section{CONTENTS}

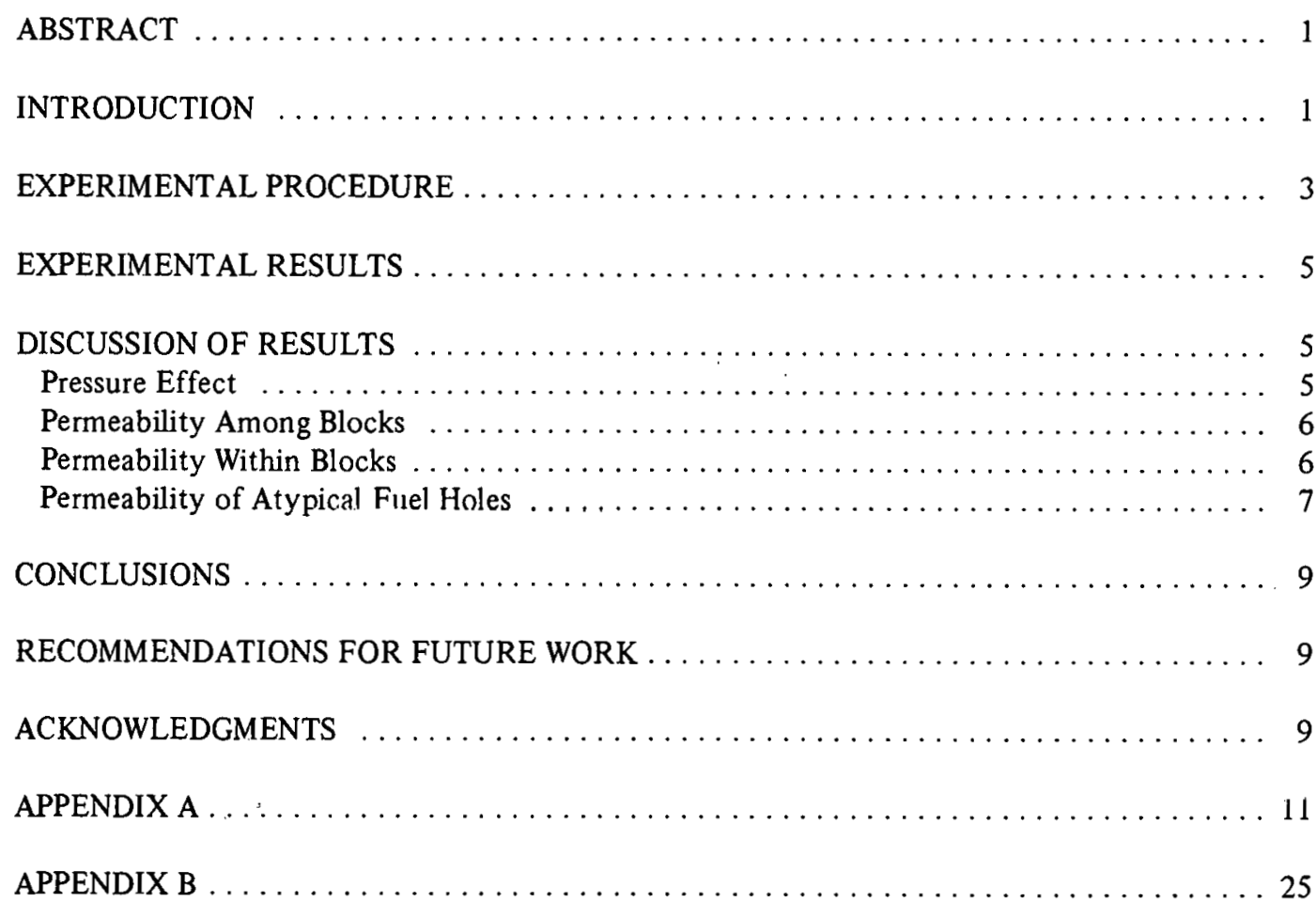




\title{
ARGON PERMEABILITY OF GRAPHITE FUEL ELEMENTS FOR THE HIGH-TEMPERATURE GAS-COOLED REACTOR
}

\begin{abstract}
A. J. Caputo, D. R. Johnson, C. K. Bayne
ABSTRACT

The flow rate of pressurized argon escaping from individual fuel holes in High-Temperature Gas-Cooled Reactor (HTGR) fuel-element blocks by diffusion through graphite webs surrounding the holes was measured for ten fuel-element blocks. The argon permeability varied significantly from block to block. Also, within the blocks, the permeability varied as a function of radial distance from the axis of the prismatic shape. The argon permeability was highest in the center of the fucl blocks. Argon permeability contours were not entirely symmetric, nor were they necessarily centered at the geometric center of the block. The argon permeabilities of fuel holes near the center and exterior of the blocks, where the web thickness is different from that of regular holes, differed from those of the regular fuel holes; however, the difference was too small to be of practical importance.
\end{abstract}

\section{INTRODUCTION}

The fuel element for the High-Temperature Gas-Cooled Reactor (HTGR) is comprised of a machined graphite block which contains the fuel rods. The graphite block is the fuel moderator and is also a structural member in the reactor core. The fuel is in the form of thoria and uranium oxide-uranium carbide microspheres that have been coated with multiple layers of pyrolytic carbon and, in the case of the fissile microspheres, silicon carbide. The fuel particles are bonded together into cylindrical rods. The prismatic graphite block has a hexagonal cross section, 14 in. across the flats and $31 \mathrm{in.} \mathrm{long.} \mathrm{The} \mathrm{fuel} \mathrm{rods} \mathrm{are} \mathrm{contained} \mathrm{in} \mathrm{blind} \mathrm{holes} \mathrm{drilled} \mathrm{parallel} \mathrm{to} \mathrm{the} \mathrm{vertical} \mathrm{axis} \mathrm{of} \mathrm{the}$ element. Coolant holes are drilled parallel to the fuel holes. Figure 1 illustrates the geometry of the fuel element; the hexagonal symmetry of the fuel and coolant hole positions is apparent. Each fuel hole is surrounded by three coolant holes, and each coolant hole is surrounded by six fuel holes. The graphite web separating the fuel from the coolant is nominally 0.178 in. thick.

The fabrication process for the fuel rods involves bonding the fuel particles together with petroleum pitch and graphite powder by warm $\left(180^{\circ} \mathrm{C}\right)$ molding. The molded rods are loaded into the fuel-element holes, and the holes are capped with graphite plugs. The elements are then heated in an inert atmosphere to $1000^{\circ} \mathrm{C}$ in order to decompose the pitch and drive off the volatile decomposition products. The element is subsequently heated to $1800^{\circ} \mathrm{C}$ in order to stabilize the fuel rods.

During the decomposition of the pitch binder, volatile decomposition products are released. Approximately $70 \%$ of the total weight of the pitch is lost as volatile reaction products. The gaseous reaction products must escape from the graphite fuel block by diffusing through the 0.178 -in.-thick wicbs separating the fuel from the inert sweep gas. The microstructure and physical and mechanical properties of the bonded fuel rods are strongly dependent on the heating rate during the carbonization process. The gas permeability of the graphite webs probably influences the process. Because the pores in graphite fuel blocks are presumably much larger than the hydrocarbon gas molecules, it was tacitly assumed that the permeability of the graphite to hydrocarbons is linearly related to the argon permeability.

The purpose of this investigation was to characterize the relative argon permeability of ten HTGR fuel-element blocks. The permeability variation within each block and among the blocks was measured. 
ORNL-DWG 70-10233R

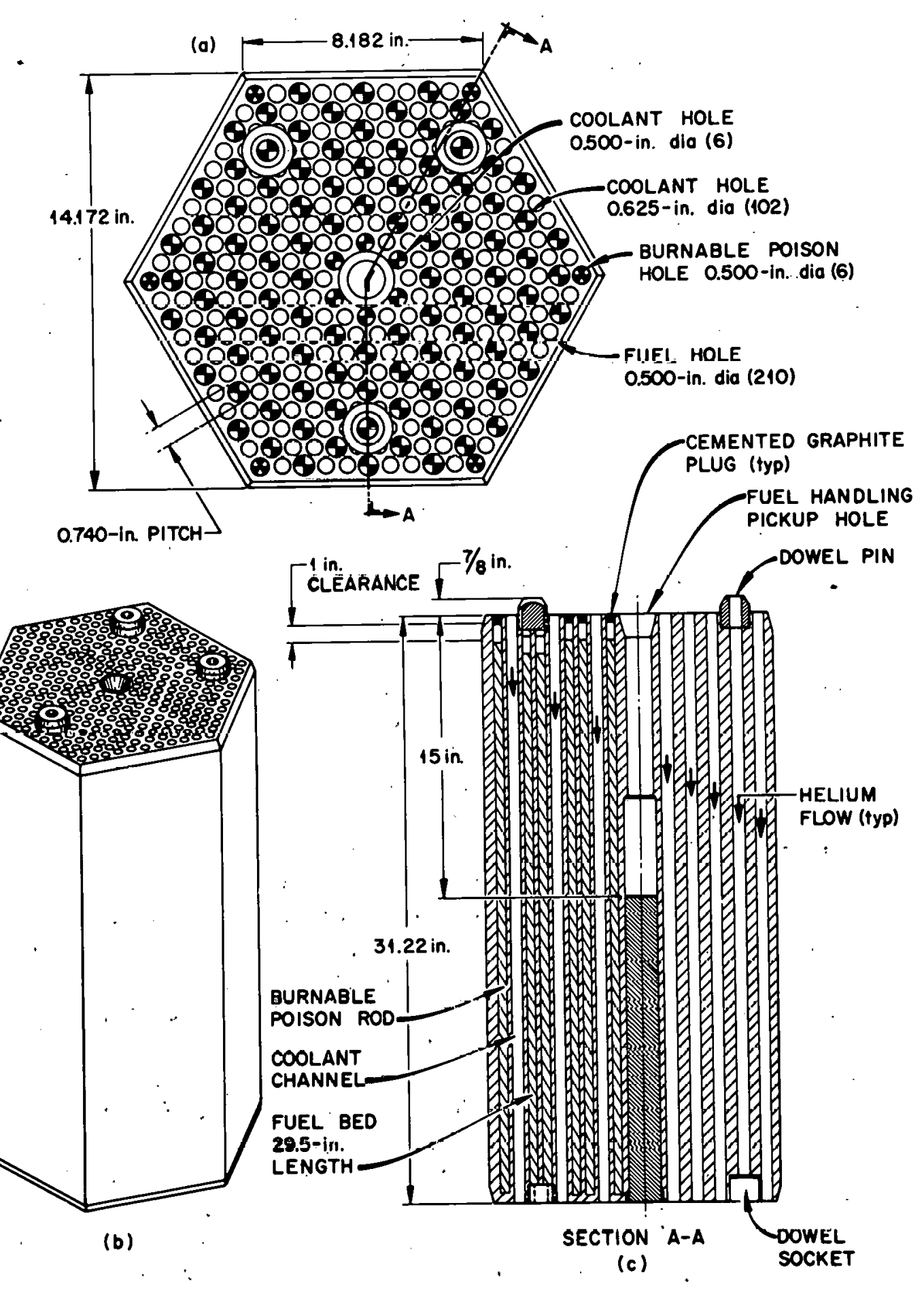

Fig. 1. H'TGR graphite fuelelement block. 


\section{EXPERIMENTAL PROCEDURE}

Ten HTGR fuel-element blocks were acquired from the General Atomic Company, San Diego, California. The blocks were the type manufactured for the Ft. St. Vrain HTGR located near Denver, Colorado. The graphite was type H-327.*

The specific argon permeability of the graphite was not determined, because the geometry of the fuel blocks was too complex. Rather, argon was introduced into the fuel holes and maintained at a fixed pressure of 4 psig at the bottom of the holes while the tops of the holes were sealed, and the resulting argon flow was measured. Four psig was chosen as a convenient value within the sensitivity range of the pressure gauges. Measurements were also made at higher and lower pressures to determine the pressure effect on the argon flow. The relative argon permeabilities are expressed as flows in liters per minute standard temperature and pressure.

Figures 2 and 3 illustrate the device constructed for relative argon permeability measurements. The probe seals the top of the fuel hole while introducing argon at a regulated pressure. Provisions were made for measuring the resulting argon pressure at the top and bottom of the fuel hole; however, no appreciable differences were seen between the pressures at the top and bottom. The argon flows were measured with rotameters. Parallel rotameters and exchangeable rotameter floats allowed the

${ }^{*} \mathrm{H}-327$ graphite is manufactured by Great Lakes Carbon Company.

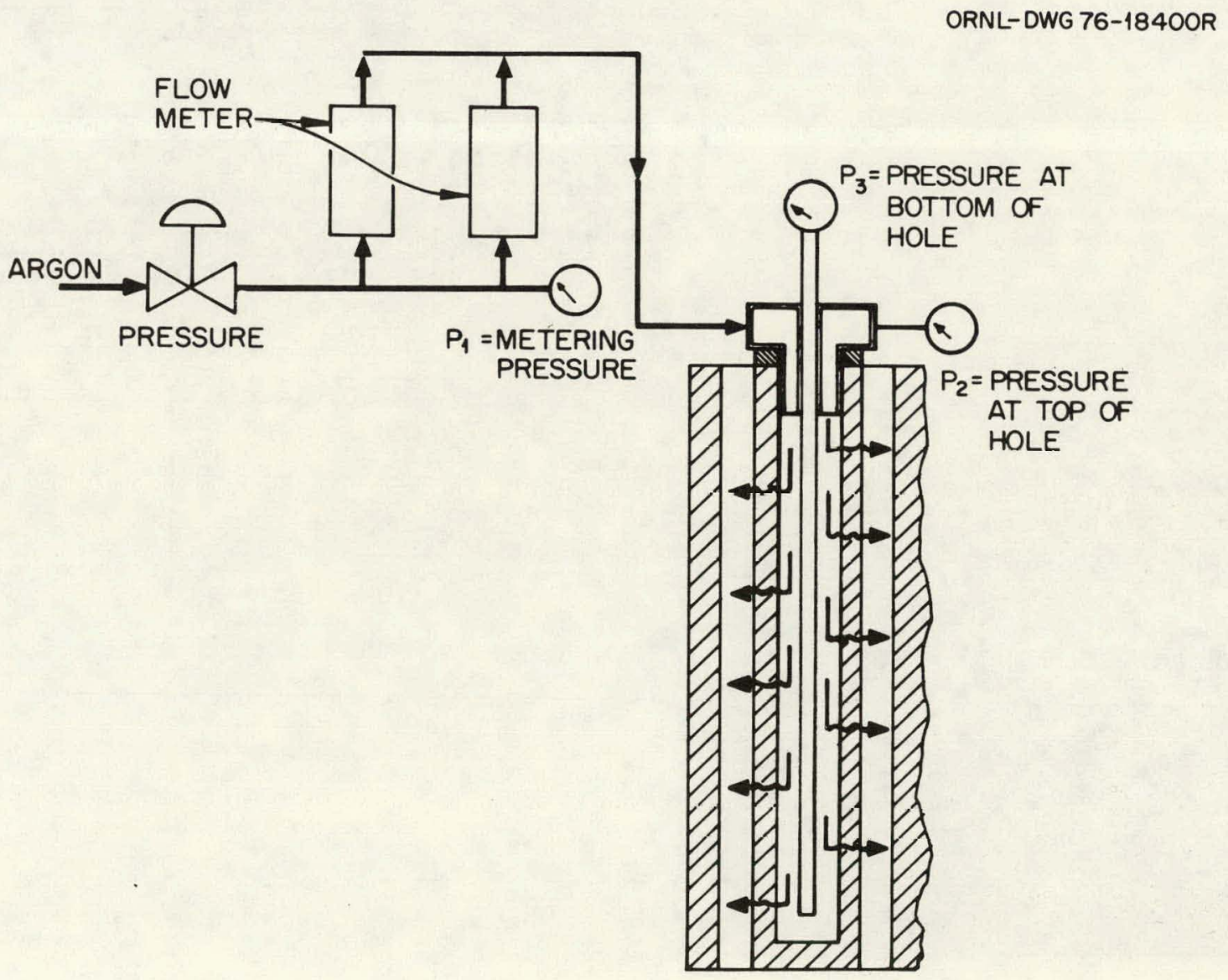

Fig. 2. Schematic drawing of argon-permeability measuring device. 


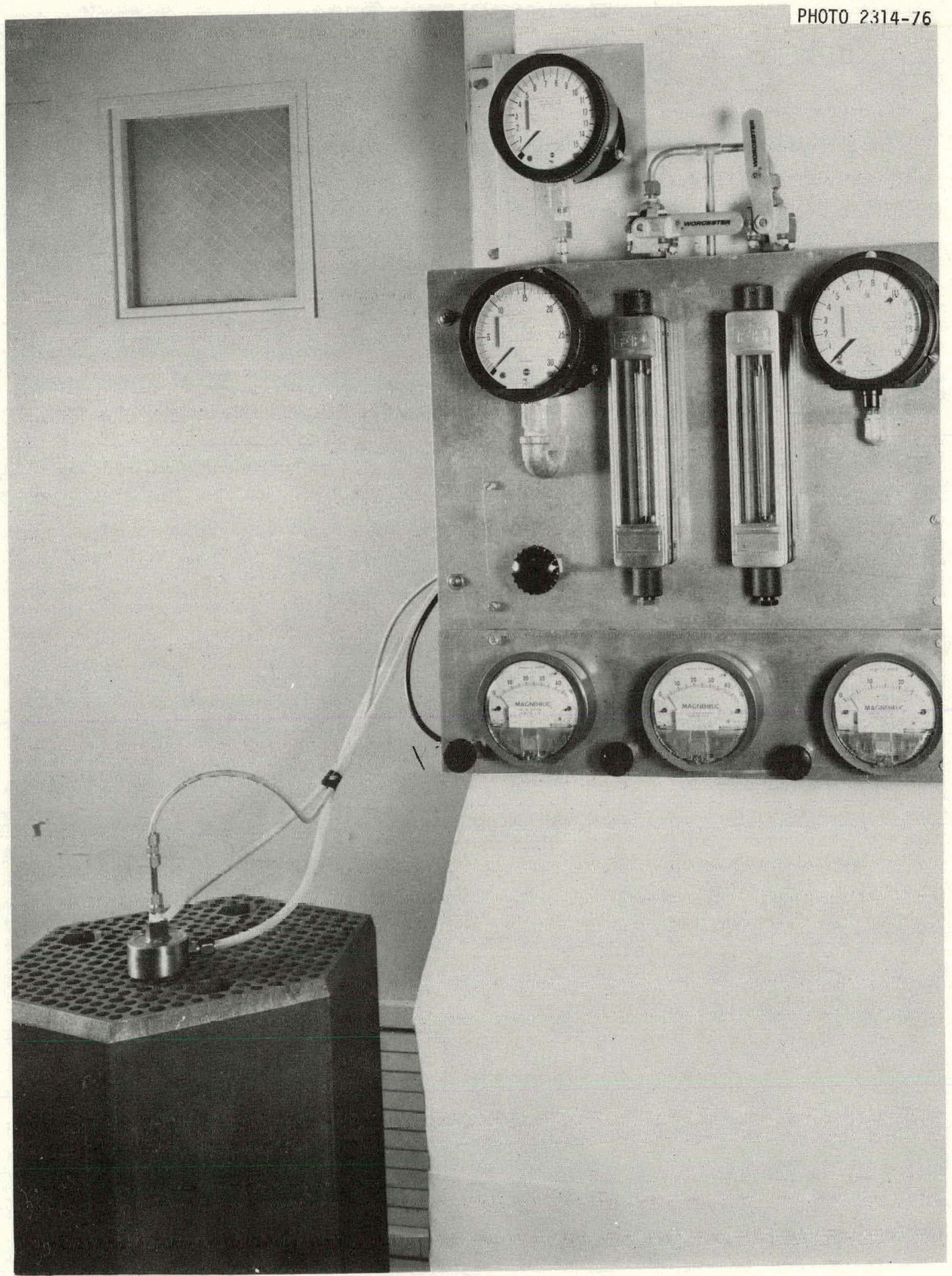

Fig. 3. Argon-permeability measuring device. 
measurement of flows ranging from 0.2 to 40.0 liters $/ \mathrm{min}$. All measurements were corrected to standard conditions, 14.7 psia and $70^{\circ} \mathrm{F}$, by using procedures and data provided by the rotameter manufacturer. ${ }^{1}$

Because the fuel-element blocks are not entirely symmetric (the dowel holes are not symmetric), it was possible to uniquely identify each hole relative to a Cartesian coordinate system with the $(0,0)$ position at the center of the block. The fuel holes in three of the six triangular block segments were examined for argon permeability. The three segments examined were equally spaced $120^{\circ}$ apart. The holes on the borders between segments were also measured.

Some of the holes have atypical geometry. They were identified as follows:

$\mathrm{I}-$ Holes on the inner circle (radius $=1.48 \mathrm{in}$.) about the origin. These holes not only are close to the center hole but also are adjacent to the innermost circle of coolant holes, which have a smaller hole diameter than the other coolant holes in the block. This results in two of the three graphite webs between each fuel hole and coolant hole being thicker, $0.240 \mathrm{in}$. compared to $0.178 \mathrm{in}$. for normal webs, for these inner circle holes.

$\mathrm{S}-$ The second circle (radius $=1.96 \mathrm{in}$.) of fuel holes about the origin. These are also near the small coolant holes but have only one thick graphite web between fuel hole and coolant hole.

$\mathrm{C}-$ The holes on the corners of the hexagonal block. These are adjacent to only one coolant hole but have access to the block surface through two 0.427 -in. webs.

F-The holes along the flat sides of the block. These are adjacent to two coolant holes but have access to the block surface through a 0.427 -in. web.

As all the atypical geometries were represented by an alphabetical letter, it was convenient to represent the typical (or regular) fuel holes by "R."

All of the holes with atypical geometry were measured in each block. In all, a total of 146 holes were measured in each block.

The results were analyzed by multiple-linear-regression analysis. The following variables were considered as possible sources of variation in the permeability results: different fuel blocks, hole position within the blocks, and the holes with special geometries.

\section{EXPERIMENTAL RESULTS}

A statistical analysis of the data by multiple linear regression is attached as Appendix A. The complete experimental data are attached as Appendix B.

\section{DISCUSSION OF RESULTS}

\section{Pressure Effect}

The effect of pressure on the permeability of porous materials, including graphite, has been reported elsewhere. ${ }^{2-4}$ For the complex geometry involved in this investigation, the overall pressure

1. Handbook Tri-Flat Variable-Area Flowmeters, Fischer and Porter Company (10 A 9010), Warminster, Pennsylvania, 1962.

2. H. P. G. Darcy, Les Fontaines publiques de la ville Dijon, Victor Dalmont, Paris, 1856.

3. P. C. Carman, Flow of Gases Through Porous Media, Academic Press, Inc., London, 1956.

4. W. P: Eatherly, et al., "Physical Properties of Graphite Materials for Special Nuclear Applications," pp. 389-401 in Peaceful Uses At. Energy Proc. Int. Conf., 2nd. 1958, Vol. 8, United Nations; Geneva; 1958. 
effect was determined by taking argon-flow measurements at pressures ranging from 0.25 to $10 \mathrm{psig}$. The measurements were made in holes that represented the lowest, average, and highest relative permeability values found in this investigation. This pressure effect is shown in Fig. 4. Thus, the results of this investigation can be extended to other pressures by estimating according to the data displayed in Fig. 4.

\section{Permeability Among Blocks}

The fuel-element blocks showed an appreciable variation in relative argon permeability among blocks. The average permeability of each block is listed in Table 1. The overall average argon flow for all holes measured in the ten blocks was 14.9 liters $/ \mathrm{min}$. The average for an individual block ranged from a low of 6.2 to a high of 25.4 liters $/ \mathrm{min}$. This variation in the average permeabilities among blocks is no doubt primarily due to differences in the porosity of the graphite logs from which the fuel blocks were made. The surface condition of the fuel holes, which is determined by drilling speed, tool sharpness, etc., may also be a factor.

\section{Permeability Within Blocks}

Significant differences in argon permeability occurred within the individual blocks. The major source of the within-block variation was a systematic radial gradient in argon permeability. The permeability was highest along the central axis of the block and decreased radially toward the outside

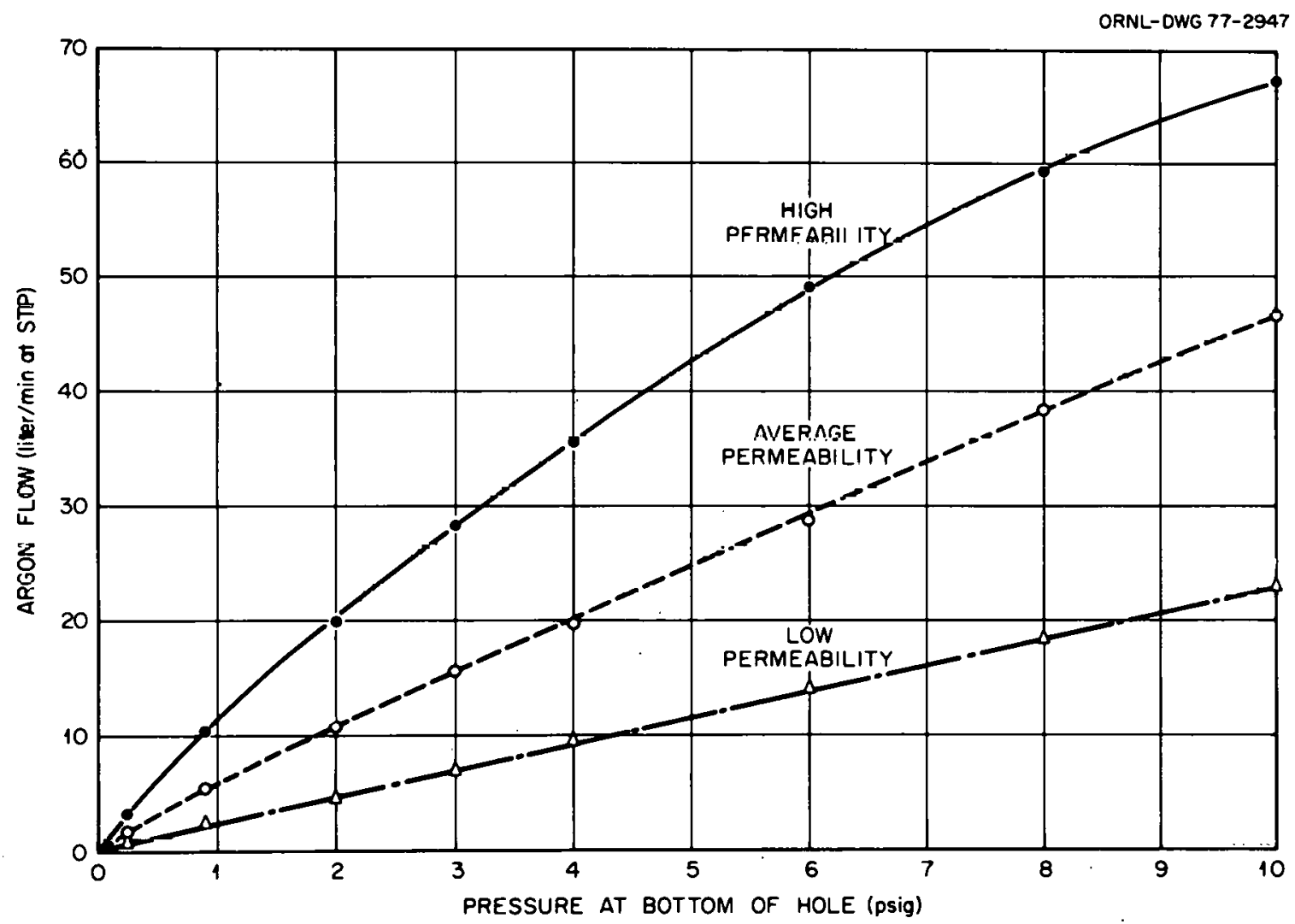

Fig. 4. Pressure effect on the argon flow from HTGR graphite fuelelement holes at various permeability levels. 
Table 1. Permeability of HTGR fuel-element blocks

\begin{tabular}{ccc}
\hline $\begin{array}{c}\text { Block } \\
\text { number }\end{array}$ & \multicolumn{2}{c}{$\begin{array}{c}\text { Relative argon flow } \\
\text { (standard liters/min) }\end{array}$} \\
\cline { 2 - 3 } & $\begin{array}{c}\text { Block } \\
\text { mean }\end{array}$ & $\begin{array}{c}\text { Range of } \\
\text { individual hules }\end{array}$ \\
\hline $1-0372$ & 6.2 & $1.5-12.9$ \\
$1-0637$ & 14.1 & $4.0-23.4$ \\
$1-1046$ & 6.7 & $0.8-16.4$ \\
$1-1343$ & 11.5 & $5.5-15.9$ \\
$1-1622$ & 11.4 & $2.3-25.6$ \\
$1-2926$ & 23.0 & $4.7-38.6$ \\
$1-4748$ & 21.4 & $20-37.1$ \\
$1-4749$ & 20.2 & $1.7-36.7$ \\
$1-5285$ & 9.1 & $1.5-22.3$ \\
$1-5864$ & 25.4 & $6.4-41.3$ \\
Overall & 14.9 & \\
mean & & \\
Overall & $6.2-25.4$ & $0.8-41.3$ \\
range & & \\
\hline
\end{tabular}

surface. Figure 5 illustrates the radial variation for block 1-0637. Presumably the radial gradient in gas permeability results from the impregnation step in the graphite fabrication. Since the pitch is intruded into the shape from the outside, one might expect the porosity of the graphite to be highest in the center and lowest on the outer surfaces. The gas permeability would be expected to be higher in the porous center of the graphite log.

Contours of equal argon permeability were calculated from the multiple-regression equations determined for the blocks. The contour plots are included in Appendix A. The argon permeability contour plots are analogous to contour land maps and can be considered as maps of the argon . permeability for each block. The outstanding feature of the contour plots is the radial permeability gradient. However, in most cases the contours are elliptical; that is, the permeability gradient is not entirely symmetrical. Also, the centers of the contour maps deviate from the geometrical centers of the blocks in most cases. A nonsymmetrical porosity gradient could result during fabrication of the graphite if, for example; the part were graphitized in a temperature gradient. This might occur if one cdgc of the part were near a furnace wall during pronressing

\section{Permeability of Atypical Fuel Holes}

The fuel holes near the center or the outside edges of the element have a different geometry for out-diffusion of gases. The argon permeabilities of the different geometry types were shown to be statistically different. The experimental data are summarized by hole type in Table 2 . The large apparent difference in hole types is mostly due to the radial effect. In lable 2 the columns, from left to right, are in order of increasing radial position. However, in the statistical analysis of the data, the permeabilities of the different hole types were calculated as though the holes were all at the same radial position. That calculation is described in Appendix A. The results are tabulated in Table A-7 of Appendix A. The effect of the hole geometry is relatively small, when adjusted for the radius effect, even though it is statistically significant. Relative to the variation in argon permeability among blocks and along the radius within blocks, the effect of hole geometry appears to be of little practical importance. 


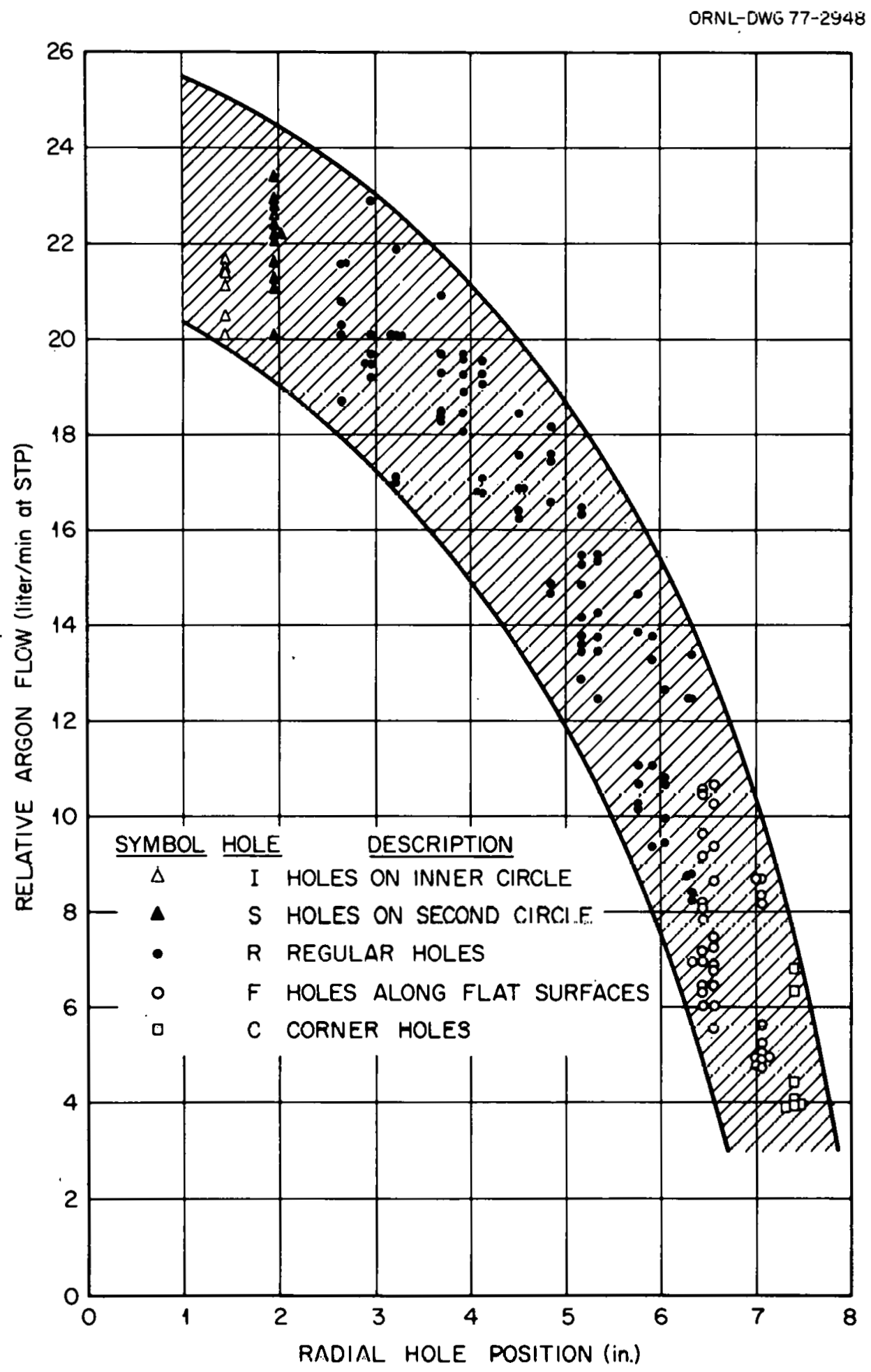

Fig. 5. Permeability of an HTGR fuel element $(1-0637)$ at 4 psig versus radial hole position. 
Table 2. Relative argon permeability of different fuel hole types in HTGR fuel element

\begin{tabular}{ccccccc}
\hline & \multicolumn{5}{c}{ Average argon flow (standard liters/min) } \\
\cline { 2 - 7 } Block & $\begin{array}{c}\text { Inner } \\
\text { No. }\end{array}$ & $\begin{array}{c}\text { Second } \\
\text { circle } \\
(1)\end{array}$ & $\begin{array}{c}\text { Regular } \\
\text { ( })\end{array}$ & $\begin{array}{c}\text { Flat } \\
\text { (F) }\end{array}$ & $\begin{array}{c}\text { Corner } \\
\text { (C) }\end{array}$ & $\begin{array}{c}\text { All } \\
\text { holes }\end{array}$ \\
\hline $1-0372$ & 9.1 & 10.7 & 6.9 & 3.0 & 2.2 & 6.2 \\
$1-0637$ & 21.1 & 22.1 & 16.0 & 7.4 & 5.0 & 14.1 \\
$1-1046$ & 11.7 & 12.2 & 7.7 & 2.6 & 1.3 & 6.7 \\
$1-1343$ & 12.9 & 13.7 & 12.7 & 8.8 & 6.3 & 11.5 \\
$1-1622$ & 17.1 & 18.4 & 12.9 & 5.7 & 3.5 & 11.4 \\
$1-2926$ & 31.4 & 34.3 & 27.0 & 11.0 & 7.0 & 23.0 \\
$1-4748$ & 31.5 & 33.6 & 24.6 & 10.5 & 5.0 & 21.4 \\
$1-4749$ & 28.3 & 30.7 & 23.8 & 9.7 & 3.4 & 20.2 \\
$1-5285$ & 18.8 & 20.3 & 10.2 & 2.4 & 1.6 & 9.1 \\
$1-5864$ & 29.5 & 33.3 & 29.2 & 15.9 & 8.0 & 25.4 \\
Average & 21.1 & 22.9 & 17.1 & 7.7 & 4.3 & 14.9 \\
\hline
\end{tabular}

\section{CONCLUSIONS}

The mean relative argon permeabilities of ten graphite HTGR fuel-element blocks were shown to differ by approximately a factor of 4 . Within the blocks the argon permeability systematically varies along the radius of the elements. The permeability along the central axis of the elements is an order of magnitude higher than at the surface.

\section{RECOMMENDATIONS FOR FUTURE WORK}

The effects of permeability on the resultant properties and irradiation behavior of carbonized fuel rods are uncertain. Property variations will be monitored during future development phases of in-block carbonization, and any related effects in Fort St. Vrain test elements will be noted.

\section{ACKNOWLEDGMENTS}

The authors wish to express their appreciation to J. C. McLaughlin for taking all the measurements, to Janice Trent for transferring the measurements to keypunch cards, to Rhonda Castleberry for typing the draft report, and to S. Bomar, J. J. Beauchamp, and E. Long for their assistance in reviewing the draft report. The manuscript was prepared for publication by the Teshnical Puhlications Department. 
THIS PAGE

WAS INTENTIONALLY

LEFT BLANK 


\section{APPENDIX A}

\section{Statistical Analysis of The Data}

The statistical analysis of the data from the ten graphite blocks considered the following variables as possible sources of systematic variation: (1) hole position in the block, (2) atypical geometry types, (3) different blocks, and (4) the interaction of geometry and blocks. The interaction effect is a manifestation of geometry types responding differently from block to block.

The different effects were studied using linear models of the form:

FLOW $=$ INDEPENDENT VARIABLES + ERROR.

The INDEPENDENT VARIABLES consist of parameters which represent the effect on the FLOW measurements of different blocks, different geometry types, and different hole positions. Parameters for the INDEPENDENT VARIABLES terms were estimated by minimizing the sum of the squared errors (SSE). The errors were assumed to be independently normally distributed random variables with zero mean and constant variance, $\sigma^{2}$. More specifically, the following notation was used to describe the permeability results with multiple-regression models:

$$
\begin{aligned}
& \mu \equiv \text { an overall average, } \\
& \beta_{\mathrm{i}} \equiv \text { effect of the } \mathrm{i} t h \text { block, } \mathrm{i}=1,2, \ldots 10, \\
& \tau_{\mathrm{j}} \equiv \text { effect of } \mathrm{j} \text { th geometry type; } \tau_{1}=\mathrm{I}, \tau_{2}=\mathrm{S}, \tau_{3}=\mathrm{R}, \tau_{4}=\mathrm{F}, \text { and } \tau_{5}=\mathrm{C}: \mathrm{j}=1,2, \ldots, 5, \\
&(\beta \chi \tau)_{\mathrm{ij}} \equiv \text { effect of the } \mathrm{j} \text { th geometry type with the } \mathrm{i} \text { th block, } \\
& \mathrm{R}_{\mathrm{k}} \equiv \text { radius of the } \mathrm{k} t h \text { hole, } \mathrm{k}=1,2, \ldots, \mathrm{n}_{\mathrm{j}}, \text { where } \mathrm{n}_{\mathrm{j}} \text { depends on geometry type: } \mathrm{n}_{1}=6, \mathrm{n}_{2}=12, \\
& \quad \mathrm{n}_{3}=86, \mathrm{n}_{4}=36, \text { and } \mathrm{n}_{\mathrm{s}}=6, \text { and } \\
& \mathrm{N}=\sum_{\mathrm{j}=1}^{S} \mathrm{n}_{\mathrm{j}}=146, \\
& \mathrm{X}_{\mathrm{k}} \equiv \text { ordinate of } \mathrm{k} t h \text { point, } \\
& \mathrm{Y}_{\mathrm{k}} \equiv \text { abscissa of the kth point. }
\end{aligned}
$$

Nine models, which are listed in Table A-1, were investigated to represent the response of FLOW. The nine models represent different combinations of the BLOCK, TYPE, and POSITION effects. For example, the coefficients of the POSITION effect in models 1 through 5 and 7 do not depend on either

Table A-1. Nine multiple-regression models used to represent the response FLOW

$$
\begin{aligned}
& \text { 1. } \mathrm{FLOW}=\mu+\rho \mathbf{R}_{\mathrm{k}}+\tau_{\mathrm{j}}+\epsilon_{\mathrm{ij}} \\
& \text { 2. FLOW }=\mu+\alpha_{11} X_{k}^{2}+\alpha_{22} Y_{k}^{2}+\tau_{j}+\epsilon_{i j} \\
& \text { 3. FLOW }=\mu+\rho \mathrm{R}_{\mathrm{k}}+\beta_{\mathrm{i}}+\tau_{\mathrm{j}}+\epsilon_{\mathrm{ijk}} \\
& \text { 4. FLOW }=\mu+\alpha_{11} X_{k}^{2}+\alpha_{22} Y_{k}^{2}+\beta_{i}+\tau_{i}+\epsilon_{i j k} \\
& \text { 5. FLOW }=\mu+\rho \mathrm{R}_{\mathrm{k}}^{2}+\beta_{\mathrm{i}}+\tau_{\mathrm{j}}+(\beta \chi \tau)_{\mathrm{ij}}+\epsilon_{\mathrm{ijk}} \\
& \text { 6. FLOW }=\mu+\rho_{\mathrm{j}} \mathrm{R}_{\mathrm{k}}^{2}+\beta_{\mathrm{i}}+\tau_{\mathrm{j}}+\left(\beta_{X} \tau\right)_{\mathrm{ij}}+\epsilon_{\mathrm{ijk}} \\
& \text { 7. FLOW }=\mu+\alpha_{11} X_{k}^{2}+\alpha_{22} Y_{k}^{2}+\alpha_{1} X_{k}+\alpha_{2} Y_{k}+\alpha_{12} X_{k} Y_{k}+\beta_{i}+\tau_{j}+(\beta \chi \tau)_{i j}+\epsilon_{i j k} \\
& \text { 8. FLOW }=\mu+\rho_{1} R_{k}^{2}+\beta_{i}+\tau_{j}+(\beta \chi \tau)_{i j}+\epsilon_{i j} \\
& \text { 9. FLOW }=\mu+\alpha_{11 \mathrm{i}} \mathrm{X}_{\mathrm{k}}^{2}+\alpha_{22 \mathrm{i}} \mathrm{Y}_{\mathrm{k}}^{2}+\alpha_{\mathrm{ii}} \mathrm{X}_{\mathbf{k}}+\alpha_{2 \mathrm{i}} \mathrm{Y}_{\mathrm{k}}+\alpha_{12 \mathrm{i}} \mathrm{X}_{\mathbf{k}} \mathrm{Y}_{\mathrm{k}}+\beta_{\mathrm{i}}+\tau_{\mathrm{j}}+\left(\hat{\beta} \chi_{\tau}\right)_{\mathrm{ij}}+\epsilon_{\mathrm{ijk}}
\end{aligned}
$$


the TYPE or BLOCK effects; while the coefficients in model 6 depend on the TYPE effect, the coefficients in models 8 and 9 depend on the BLOCK effect. The pertinent statistics for the analyses of the response models are shown in Table A-2. The best model was selected by comparing the different SSEs, and their degrees of freedom (DF = number of data points minus number of parameters in the model). ${ }^{\prime}$ An estimate of the error variance is the error mean square (MSE $=$ SSE/DF), and the coefficient of determination, $\mathrm{R}^{2}$, is the precentage attributable to the response model of the total sum of squares adjusted for the mean. As more independent variables were added to the response model, the sum of squares due to error decreased. Whether the decrease was statistically significant for models I and II can be tested by comparing the F statistic,

$$
\mathrm{F}=\left[\left(\mathrm{SSE}_{\mathrm{I}}-\mathrm{SSE}_{\mathrm{II}}\right) /\left(\mathrm{DF}_{\mathrm{I}}-\mathrm{DF}_{\mathrm{II}}\right)\right] / \mathrm{MSE}_{\mathrm{II}},
$$

with the $\alpha$-percentile values of an $F$ distribution with $\nu F_{I}-D F_{I I}$ and $D F_{\text {II }}$ degrees of freedom. For example, model 9 has 40 more parameters than model 8 , and the value of the $F$ statistic is $F=34.61$, which is significant even at the $0.5 \%$ significance level.

Because model 9 had the lowest SSE and was significantly different from any other model, it was chosen as the best model. The total sum of squares can be partitioned among the independent variables of model 9 as shown in the analysis of variance table, Table A-3 (see ref. 2). The order in which a source of variation appears in the analysis of variance table means that its sum of squares has been adjusted for those sources listed above its position. For example, the TYPE source has had its sum of squares adjusted for both BLOCK and POSITION (terms in the model involving the $\mathrm{x}$ and $\mathrm{y}$ coordinates) effects. The analysis of variance table (Table A-3) shows that after the fixed effect!variables BLOCK, TYPE, and BLOCK $\times$ TYPE have been adjusted for the covariate effect of POSITION, the fixed effect variables have $F$ values, where $F=$ (MS for a source)/(MS for error), which are significant even at very low significance levels. If the order of the variables in the regression model is changed so that POSITION is adjusted for the fixed effects, the sum of squares can be partitioned as in Table A-4. The POSITION effect is significant at small significance levels even when adjusted for all the fixed

1. N. R. Draper and H. Smith, Applied Regression Analysis, John Wiley and Sons, Inc., New York, N.Y., 1966.

2. G. W. Snedicor and W. G. Cochran, Statistical Methods, Iowa State University Press, Ames, Iowa, 1967.

Table A-2. Statistics for the nine multiple-regression models

\begin{tabular}{crrrr}
\hline Model & SSE & DF & MSE & $\mathrm{R}^{2}$ \\
\hline 1 & 86063.53 & 1454 & 59.19 & $38 \%$ \\
2 & 85727.93 & 1453 & 59.00 & $38 \%$ \\
3 & 20728.86 & 144.5 & 14.35 & $85 \%$ \\
4 & 20389.51 & 1444 & 14.12 & $85 \%$ \\
5 & 12224.48 & 1409 & 8.68 & $91 \%$ \\
6 & 12214.56 & 1405 & 8.69 & $91 \%$ \\
7 & 12127.36 & 1405 & 8.63 & $91 \%$ \\
8 & 9929.18 & 1400 & 7.09 & $93 \%$ \\
9 & 4917.29 & 1360 & 3.62 & $96 \%$ \\
\hline
\end{tabular}

Table A-3. Analysis of variance for model 9

\begin{tabular}{lrrrr}
\hline \multicolumn{1}{c}{ Source } & D.F. & \multicolumn{1}{c}{ S.S. } & M.S. & F value \\
\hline Position & 50 & 90121.10 & 1802.42 & 497.91 \\
Block & 9 & 41019.31 & 4557.70 & 1260.55 \\
Geometry type & 4 & 934.94 & 233.74 & 64.57 \\
Block $\times$ type & 36 & 1534.76 & 42.63 & 11.78 \\
Error & 1360 & 4217.29 & 3.62 & \\
$\quad$ Total & 1459 & 138527.40 & & \\
\hline
\end{tabular}


Table A-4. Alternative analysis of variance for model 9

\begin{tabular}{lrrrr}
\hline \multicolumn{1}{c}{ Source } & D.F. & \multicolumn{1}{c}{ S.S. } & \multicolumn{1}{c}{ M.S. } & F value \\
\hline Block & 9 & 65327.48 & 7258.61 & 2005.14 \\
Type & 4 & 39618.87 & 9904.72 & 2736.11 \\
Block $\times$ type & 36 & 8163.91 & 226.78 & 62.65 \\
Position & 50 & 20499.85 & 410.00 & 113.26 \\
Error & 1360 & 4917.29 & 3.62 & \\
$\quad$ Total & 1459 & 138527.40 & & \\
\hline
\end{tabular}

effects. Considering both analysis of variance tables (Tables A-3 and A-4), we can conclude that the four sources, POSITION, BLOCK, TYPE, and BLOCK $\times$ TYPE, are significant sources of variation for the permeability measurements.

In order to study the POSITION and TYPE effects in more detail, the permeability data were analyzed separately for each block. The following model 10 was used:

$$
\text { FLOW }=\mu+\alpha_{11} X_{k}^{2}+\alpha_{22} Y_{k}^{2}+\alpha_{1} X_{k}+\alpha_{2} Y_{k}+\alpha_{12} X_{k} Y_{k}+\tau_{j}+\epsilon_{\mathrm{kj}},
$$

where

$$
\begin{array}{r}
\mathrm{j}=1,2, \ldots, 5\left(\tau_{1}=\mathrm{I}, \tau_{2}=\mathrm{S}, \tau_{3}=\mathrm{R}, \tau_{4}=\mathrm{F} \text {, and } \tau_{5}=\mathrm{C}\right), \\
\mathrm{k}=1,2, \ldots, \mathrm{n}_{\mathrm{j}}\left(\mathrm{n}_{1}=6, \mathrm{n}_{2}=12, \mathrm{n}_{3}=86, \mathrm{n}_{4}=36, \text { and } \mathrm{n}_{5}=6\right)
\end{array}
$$

Those POSITION parameters which were not significant at the $10 \%$ significance level under the normality assumption for the error random variables were eliminated from the model. The estimated parameter values were then recalculated. The estimated $\alpha$ coefficients for model 10 are denoted by $a$ 's, and these estimated coefficients are listed in Table A-5. A summary of some statistics for model 10 are given in Table A-6.

Model $10 \mathrm{can}$ be used for calculating contours of equal air permeability for each of the ten blocks, by using the coefficients in Table A-5. Permeability contours are shown in Figs. A-1-A-10. The contours give a picture of the permeability as a function of hole location. The holes in the center of the blocks have

Table A-5. Multiple-regression coefficients for model 10

\begin{tabular}{llllrrr}
\hline & & \multicolumn{5}{c}{ Coefficient of - } \\
\cline { 2 - 7 } Block & Intercept & \multicolumn{1}{c}{$\mathrm{Y}^{2}$} & \multicolumn{1}{c}{$\mathrm{X}$} & \multicolumn{1}{c}{$\mathrm{Y}$} & $\mathrm{XY}$ \\
\hline & & $\mathrm{X}^{2}$ & \multicolumn{1}{c}{$\mathrm{C}$} & \\
\hline $1-0372$ & 12.67 & -0.25 & -0.23 & 0.00 & 0.00 & 0.03 \\
$1-0637$ & 22.93 & -0.32 & -0.34 & 0.17 & -0.09 & -0.04 \\
$1-1046$ & 12.78 & -0.21 & -0.25 & 0.40 & -0.21 & -0.06 \\
$1-1343$ & 14.40 & -0.10 & -0.11 & -0.06 & 0.17 & 0.00 \\
$1-1622$ & 18.21 & -0.28 & -0.22 & -0.29 & 0.17 & 0.06 \\
$1-2926$ & 37.66 & -0.56 & -0.53 & -0.21 & 0.15 & 0.00 \\
$1-4748$ & 35.41 & -0.52 & -0.52 & -0.94 & -0.26 & 0.05 \\
$1-4749$ & 36.21 & -0.60 & -0.58 & 0.64 & -0.21 & -0.04 \\
$1-5285$ & 22.01 & -0.47 & -0.47 & -0.39 & 0.12 & 0.00 \\
$1-5864$ & 36.05 & -0.38 & -0.41 & 0.14 & -0.16 & 0.07 \\
\hline
\end{tabular}

Table A-6. Summary statistics for model 10 in each block

\begin{tabular}{rrrrr}
\hline Block & SSE & DF & MSE & $\mathrm{R}^{2}$ \\
\hline $1-0372$ & 146.48 & 138 & 1.06 & $90 \%$ \\
$1-0637$ & 158.41 & 136 & 1.16 & $97 \%$ \\
$1-1046$ & 251.32 & 136 & 1.85 & $91 \%$ \\
$1-1343$ & 138.12 & 137 & 1.01 & $85 \%$ \\
$1-1622$ & 324.33 & 136 & 2.38 & $92 \%$ \\
$1-2926$ & 715.07 & 137 & 5.22 & $95 \%$ \\
$1-4748$ & 401.13 & 136 & 2.95 & $97 \%$ \\
$1-4749$ & 749.05 & 136 & 5.51 & $95 \%$ \\
$1-5285$ & 669.81 & 137 & 4.89 & $91 \%$ \\
$1-5864$ & 1378.39 & 136 & 10.14 & $87 \%$ \\
\hline
\end{tabular}


ORNL-DWG-76-19452

$\begin{array}{cc}\text { Symbol } & \begin{array}{c}\text { Contour } \\ \text { Values } \\ \text { (liter/min) }\end{array} \\ 1 & 1.0 \\ 2 & 4.0 \\ 3 & 6.0 \\ 4 & 9.0 \\ 5 & 12.0\end{array}$

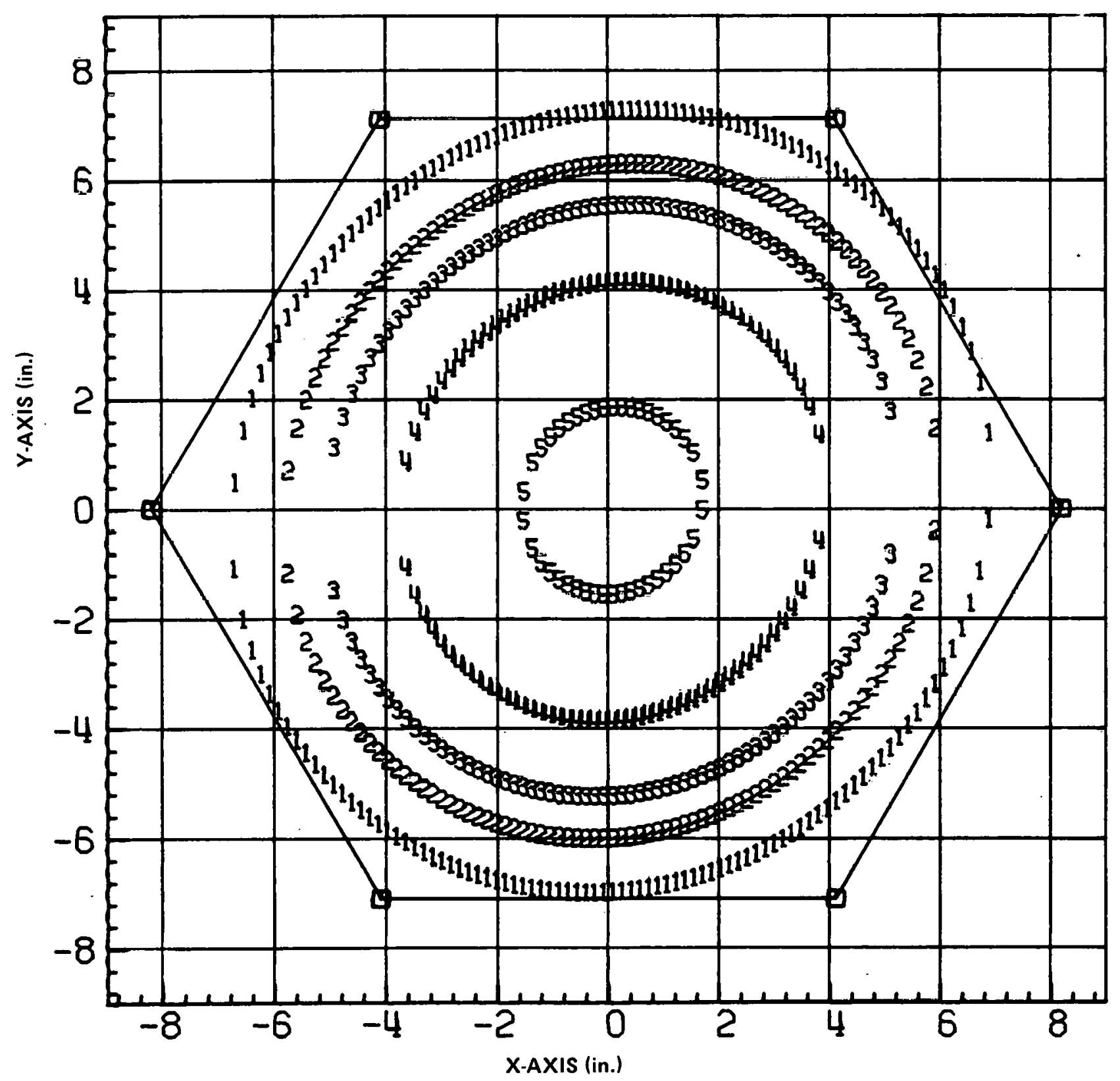

Fig. A-1. Contour plot of argon permeability for block 1-0372. 
ORNL-DWG-76-19453

$\begin{array}{cr}\text { Symbol } & \begin{array}{r}\text { Conto } \\ \text { Values } \\ \text { (liter/m }\end{array} \\ 1 & 4.0 \\ 2 & 8.0 \\ 3 & 12.0 \\ 4 & 18.0 \\ 5 & 22.0\end{array}$

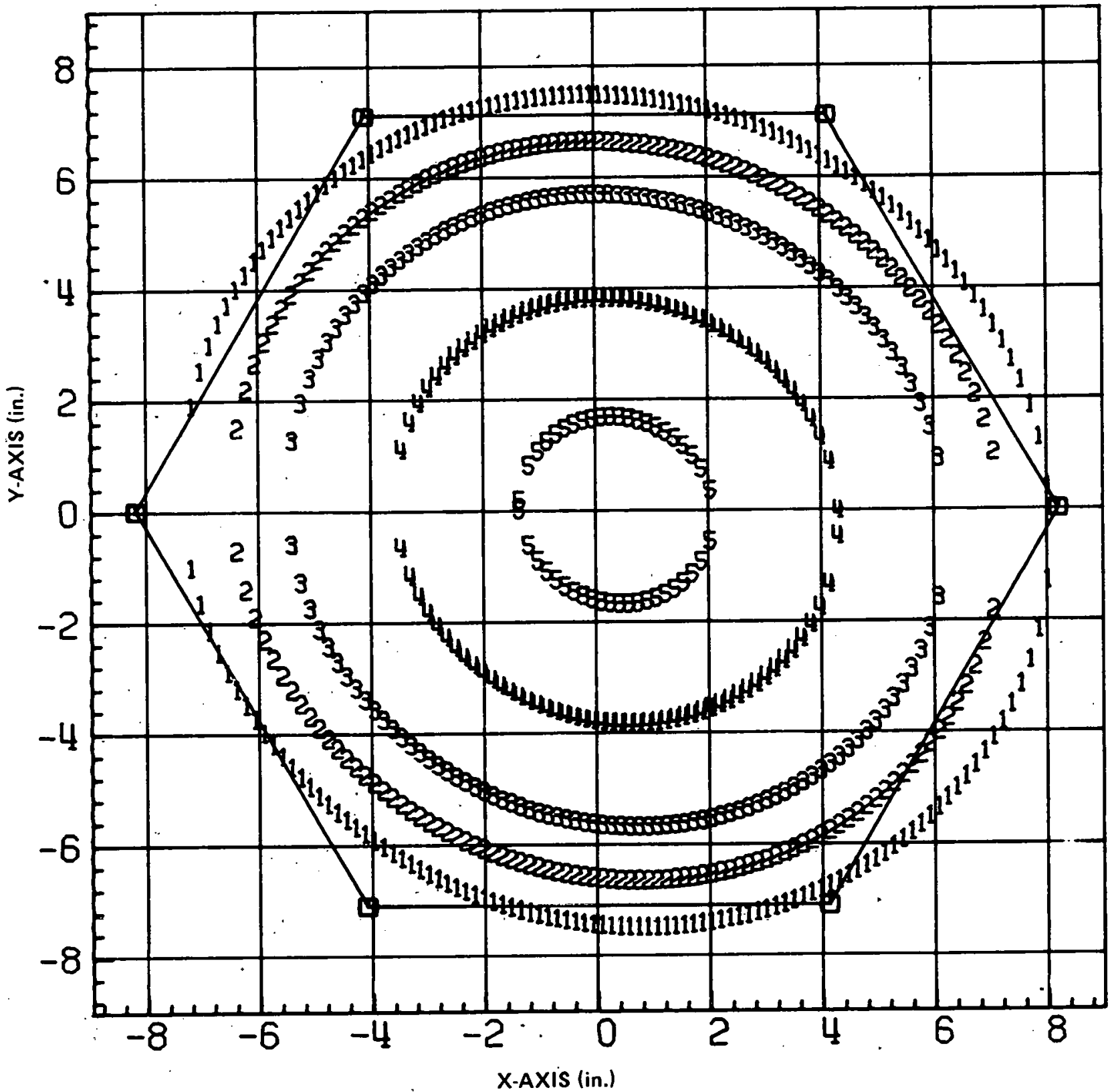

Fig. A-2. Contour plot of argon permeability for block 1-0637. 


$\begin{array}{cc}\text { Symbol } & \begin{array}{c}\text { Contour } \\ \text { Values } \\ \text { (liter/min) }\end{array} \\ 1 & 1.0 \\ 2 & 4.0 \\ 3 & 8.0 \\ 4 & 10.0 \\ 5 & 12.0\end{array}$

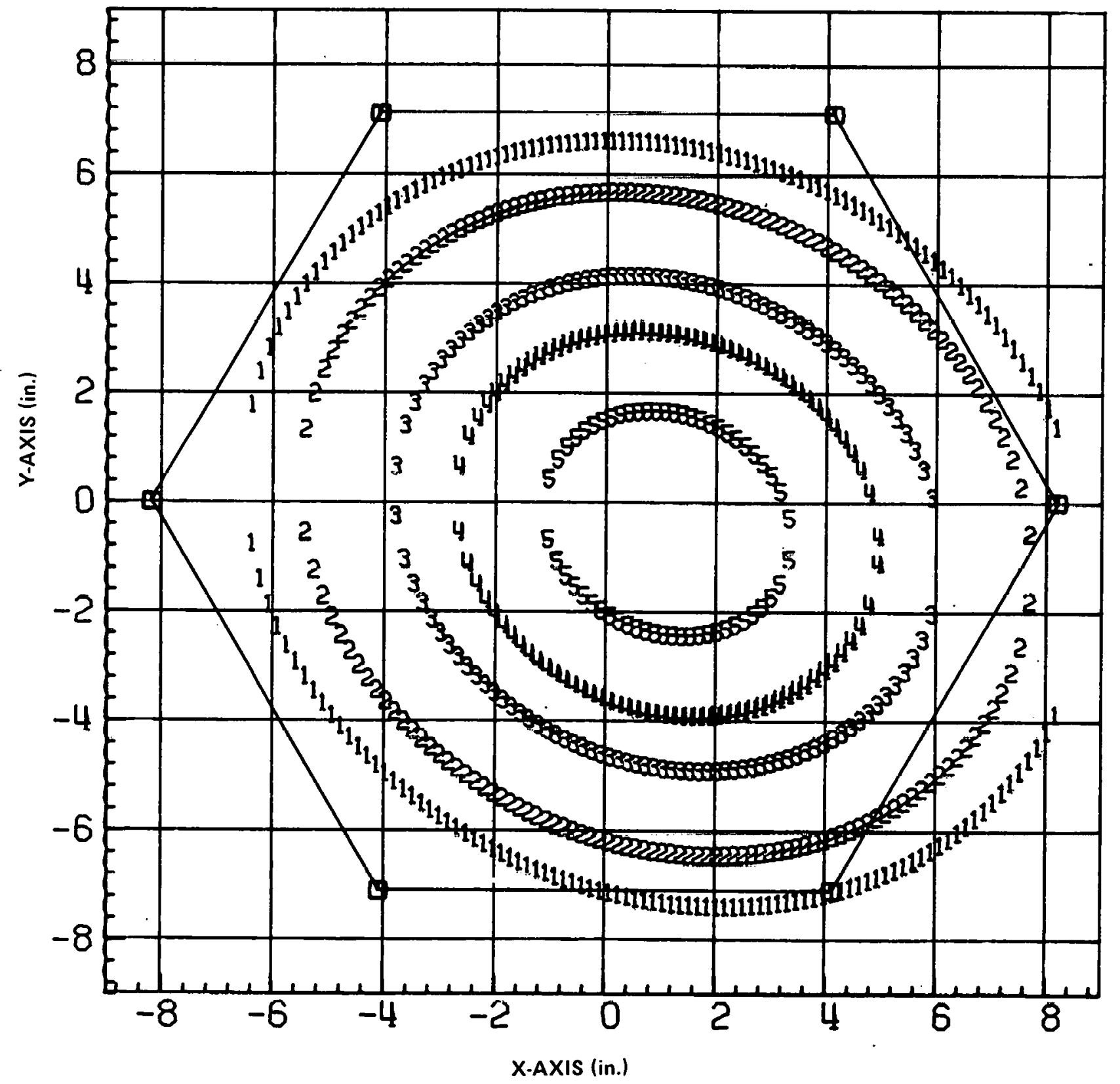

Fig. A-3. Contour plot of argon permeability for block 1-1046. 


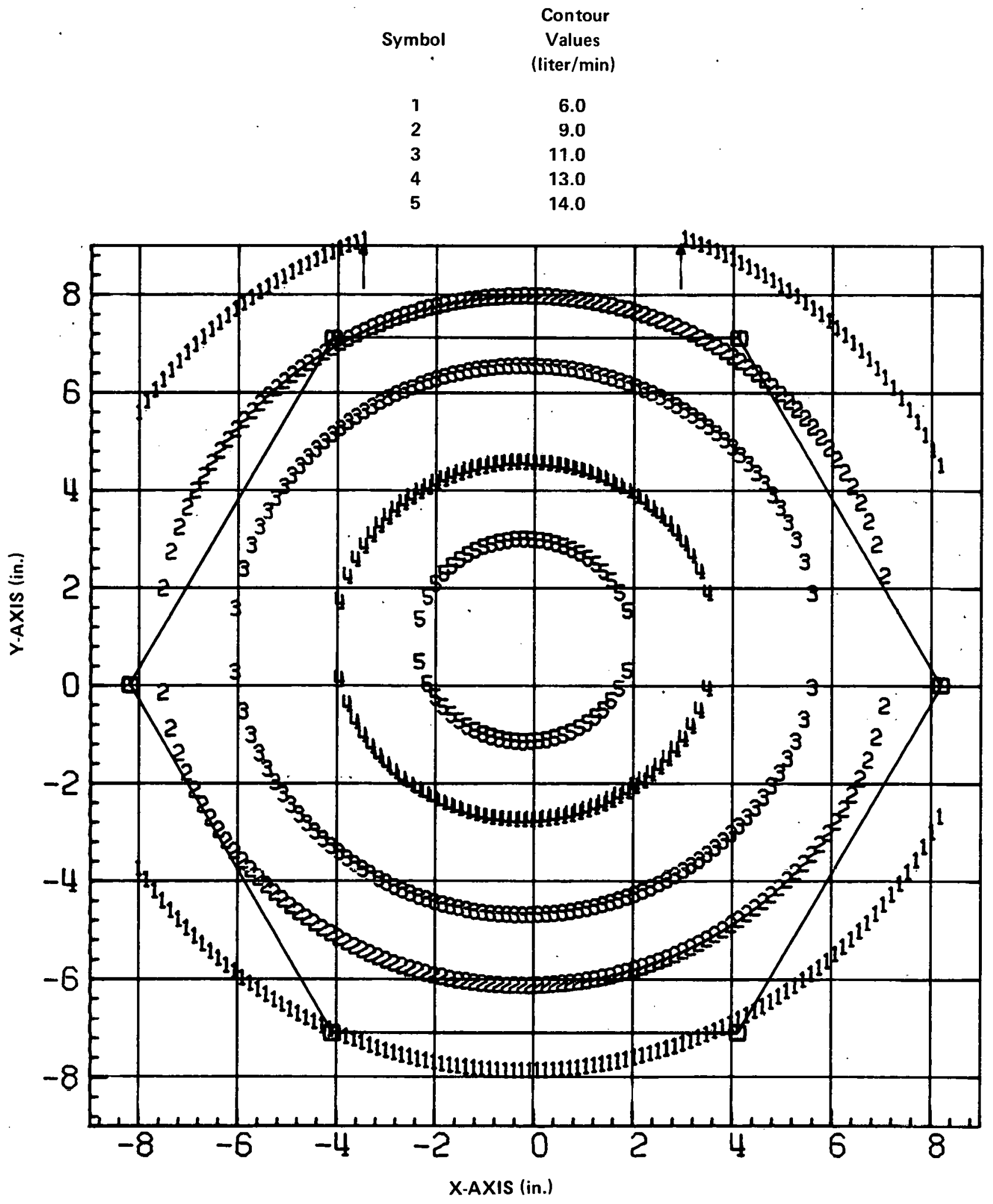

Fig. A-4. Contour plot of argon permeability for block 1-1343, 
ORNL-DWG-76-19456

$\begin{array}{cc}\text { Symbol } & \begin{array}{c}\text { Contour } \\ \text { Value } \\ \text { (liter/min) }\end{array} \\ 1 & 5.0 \\ 2 & 9.0 \\ 3 . & 13.0 \\ 4 & 16.0 \\ 5 & 18.0\end{array}$

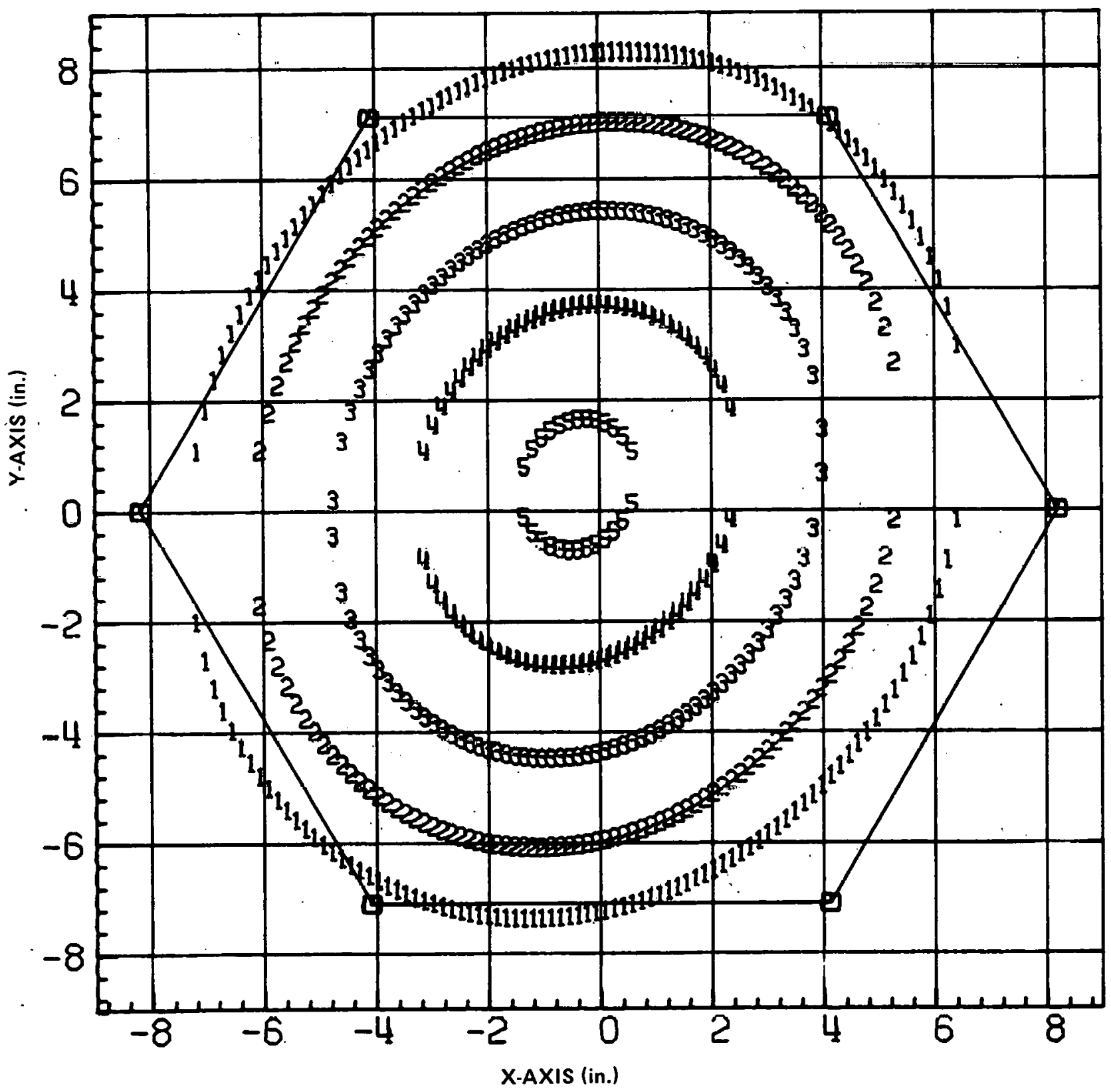

Fig. A-5. Contour plot of argon permeability for block -1-1622. 


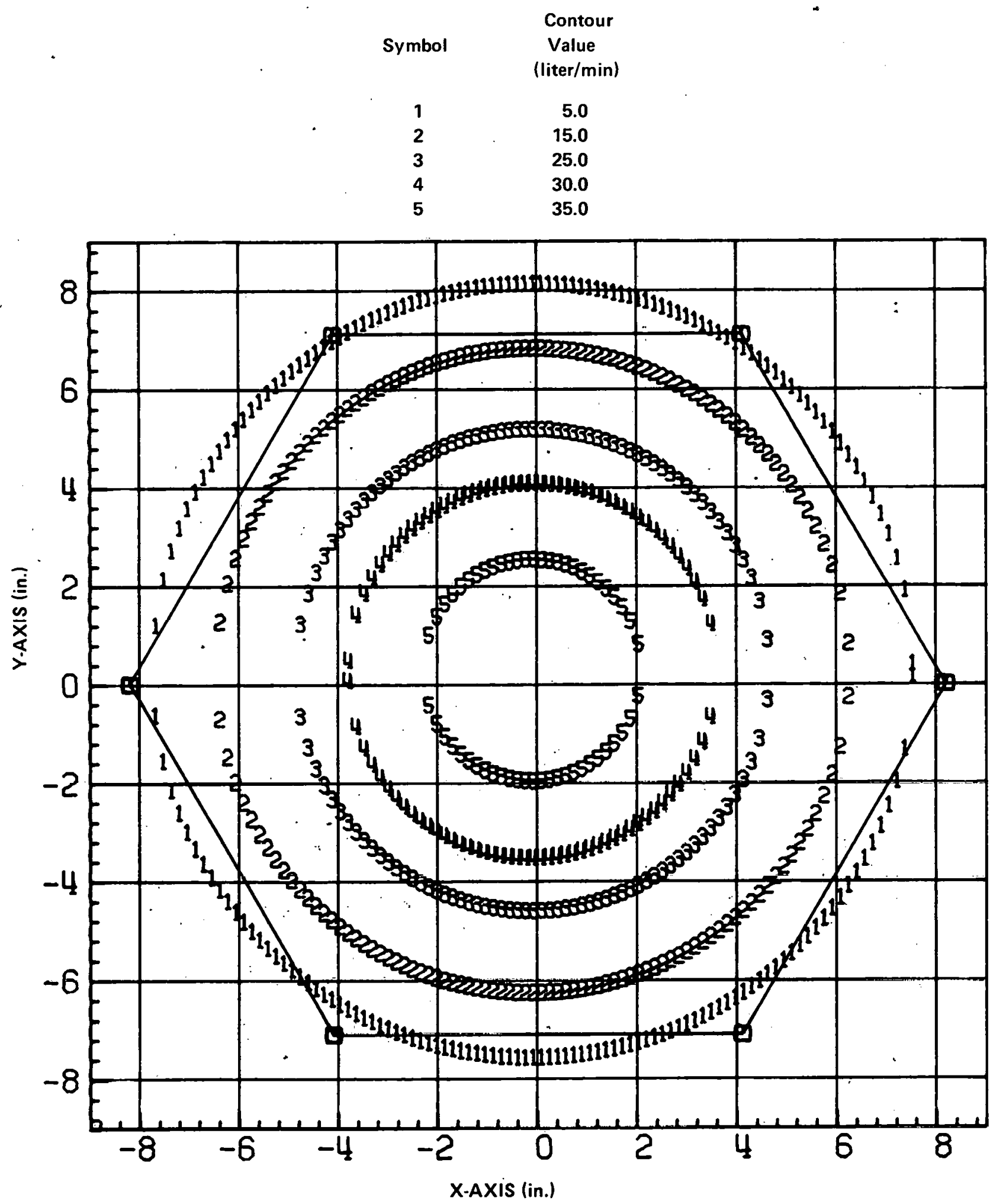

Fig. A-6. Contour plot of argon permeability for block 1-2926. 
ORNL-DWG.76-19458

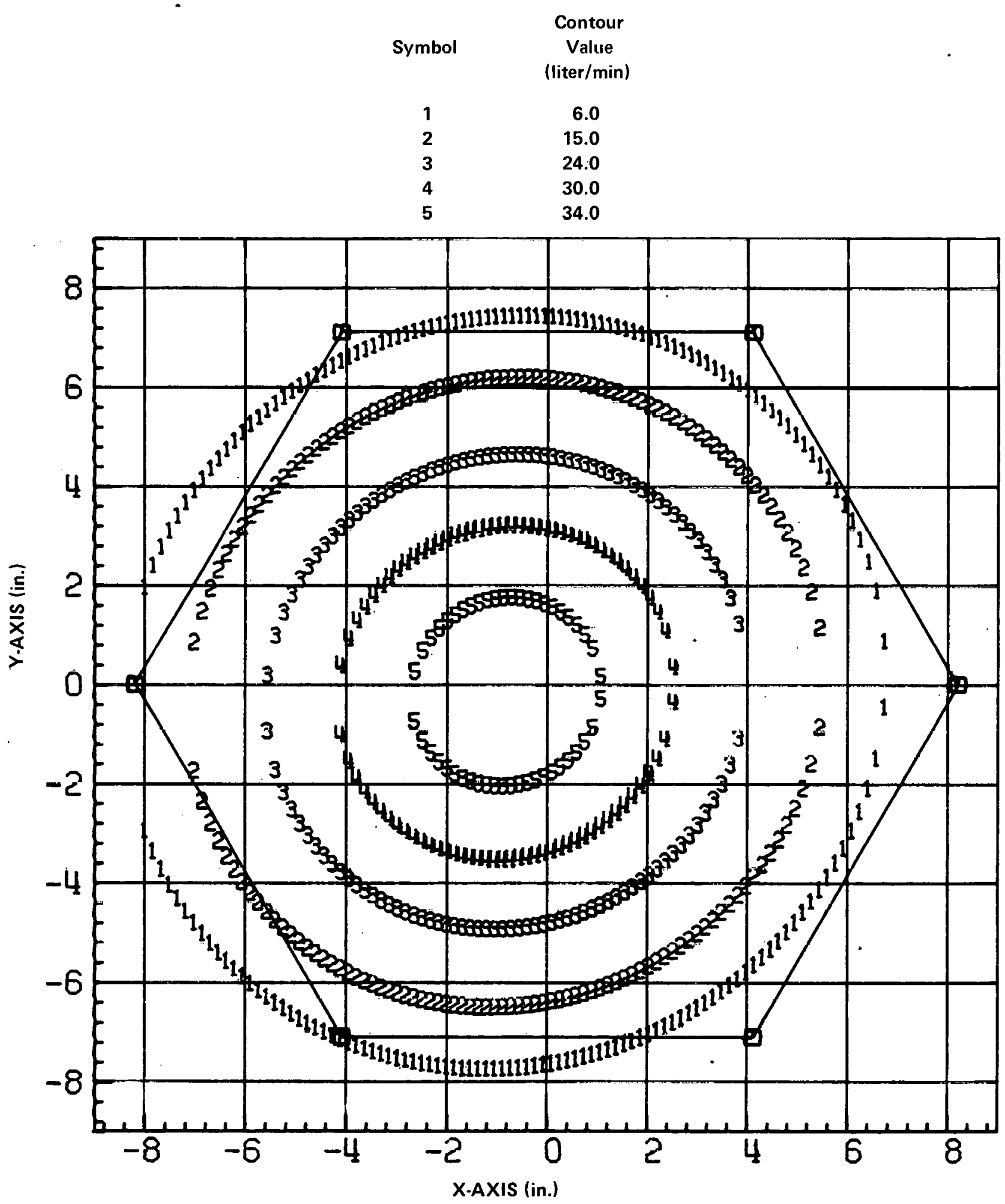

Fig. A.7. Contour plot of argon permeability for block 1.4748 . 


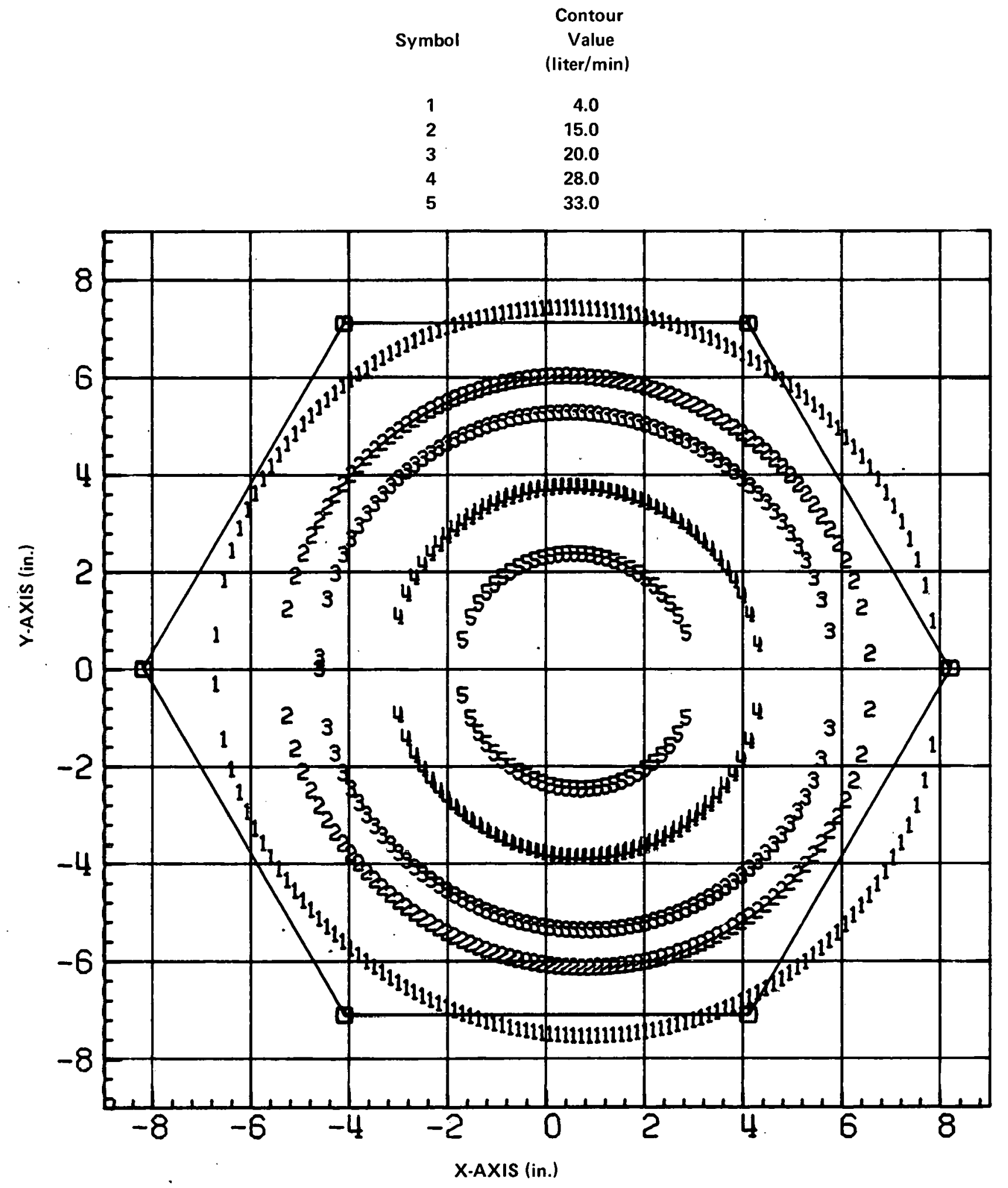

Fig. A-8. Contour plot of argon permeability for block 1-4749, 
ORNL-DWG-76-19460

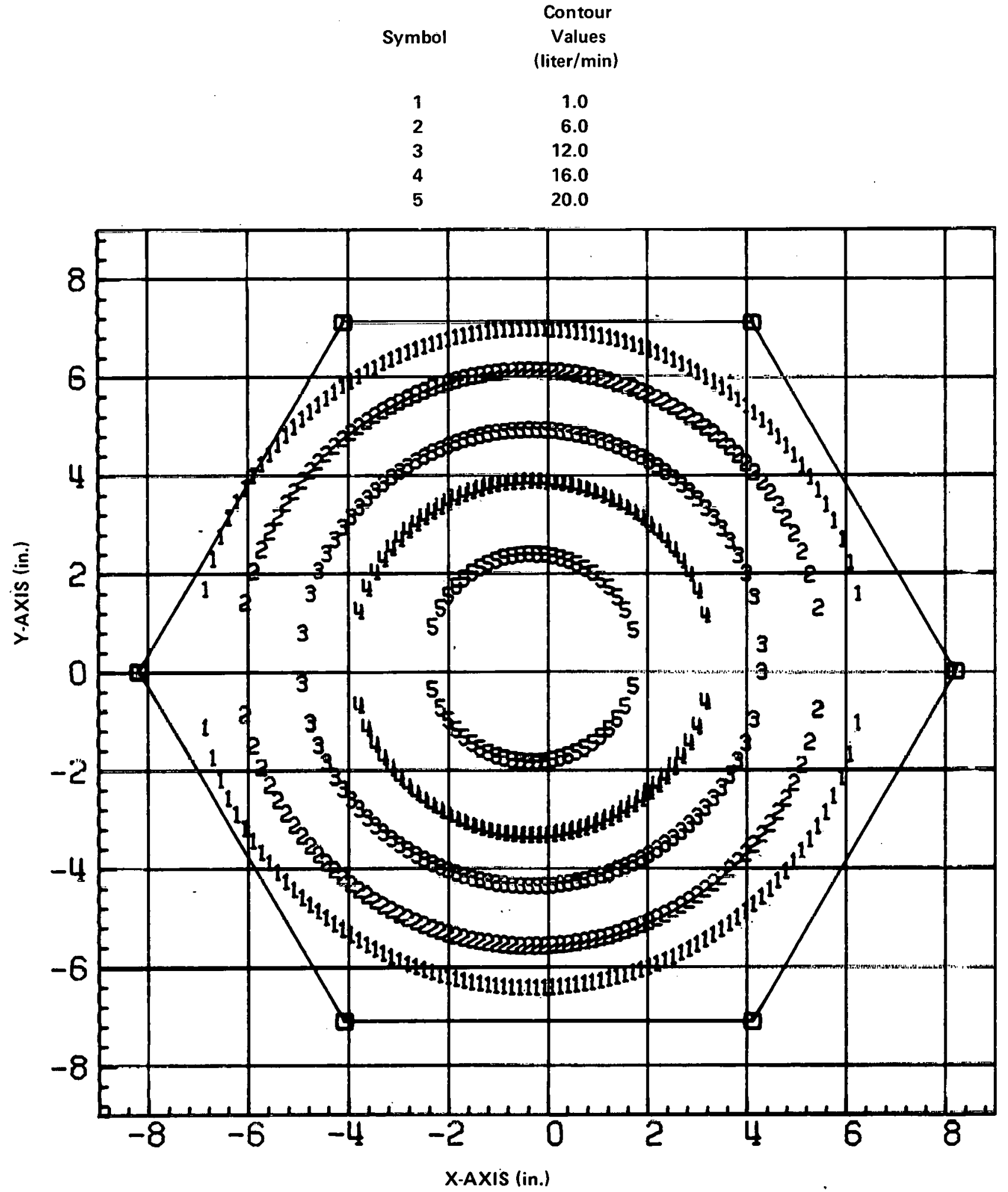

Fig. A-9. Contour plot of argon permeability for block $\mathbf{1 - 5 2 8 5}$. 
ORNL-DWG-76-19461

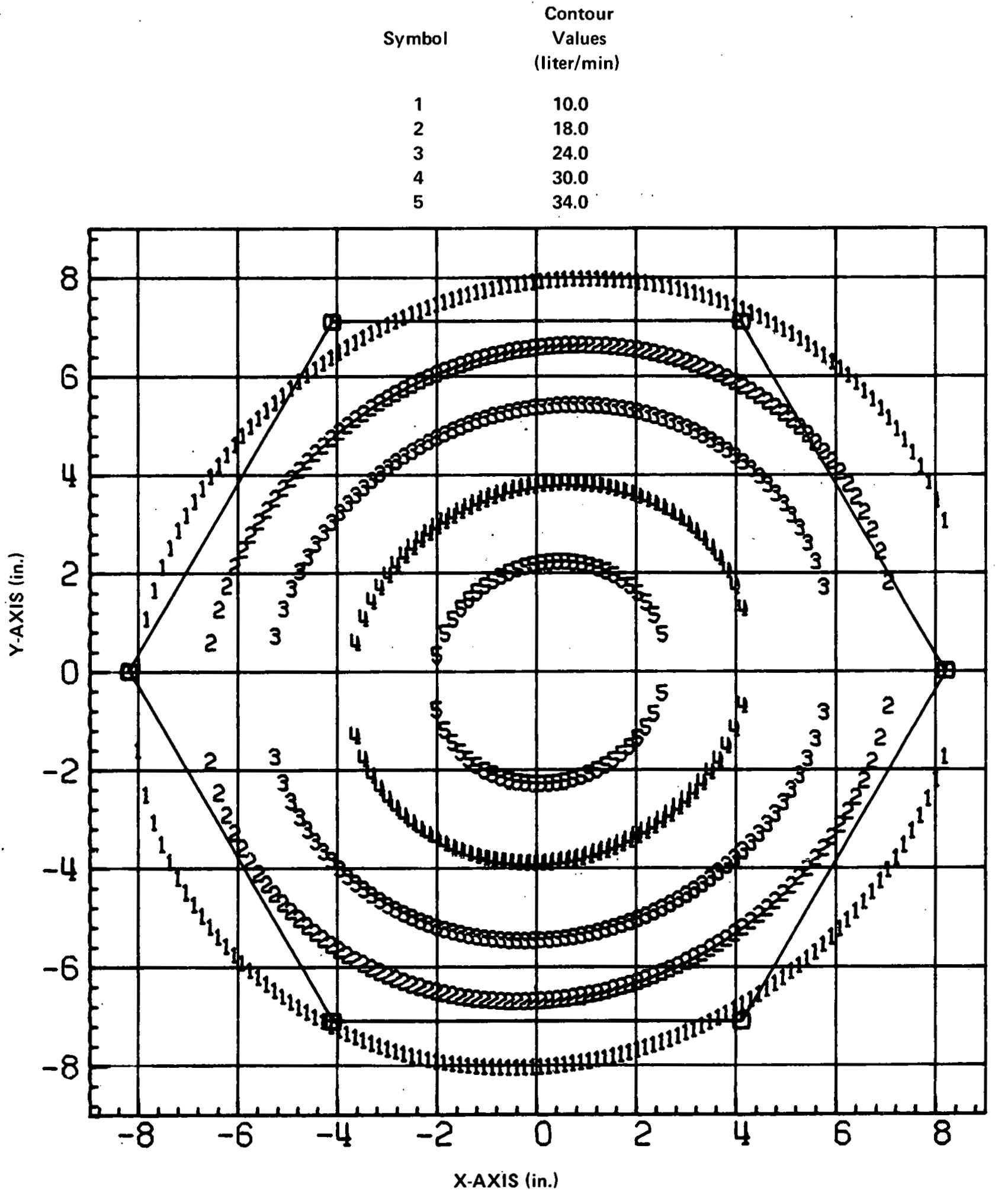

Fig. A-10. Contour plot of argun perimieability for block 1-5864. 
a higher permeability than those located near the outside. In general, the contours are slightly elliptical in shape and are not centered on the middle of the block.

The fixed effects $\tau_{1 \mathrm{j}}$ cannot be estimated with a linear combination of the responses. However, the effect $\mu+\tau_{\mathrm{j}}$ can be estimated by:

$$
\mu \hat{+} \tau_{\mathrm{j}}=\overline{Z_{\mathrm{j}}}-\mathrm{a}_{11} \overline{\mathrm{X}_{\mathrm{j} .}^{2}}-\mathrm{a}_{22} \overline{\mathrm{Y}_{\mathrm{j} .}^{2}}-\mathrm{a}_{1} \overline{\mathrm{X}_{\mathrm{j}}}-\mathrm{a}_{2} \overline{\mathrm{Y}_{\mathrm{j} .}}-\mathrm{a}_{12}(\overline{\mathrm{XY}})_{\mathrm{j} .},
$$

where $\bar{Z}_{\mathrm{i}}$. is the average of the permeability measurements over the jth geometry type, the "." notation means summed, and the "-" notation means average; for example,

$$
\overline{X_{j}^{2}}=\frac{1}{n_{j}} \sum_{k=1}^{n_{j}} X_{i k}^{2}:
$$

The estimate $\mu \hat{+} \tau_{\mathrm{j}}$ represents the sum of the overall mean and the effect of the jth geometry type adjusted for the POSITION effect. These adjusted means are tabulated in Table A-7. Differences among the geometry types within a block are significant at the 5\% level in nine blocks but not in block 1-1046. A significant difference among the geometry types means that there are two or more geometry types in a block which have a significantly different effect on the permeability response. These adjusted means represent the permeability values which would occur due to the geometry type if there were no position effect.

Table A-7. Estimates of the adjusted genmetry type means;

\begin{tabular}{|c|c|c|c|c|c|}
\hline Block & $\underline{I}$ & $S$ & $\mathbf{R}$ & $F$ & $\dot{C}$ \\
\hline $1-0372$ & 9.67 & 11.64 & 12.34 & 13.84 & 15.37 \\
\hline $1-0037$ & 21.77 & 23.31 & 23.33 & 22.014 & 22.84 \\
\hline $1-1046$ & 12.17 & 13.11 & 12.73 & 12.73 & 13.65 \\
\hline $1-1343$ & 13.16 & 14.13 & 15.07 & 13.49 & 12.10 \\
\hline $1-1622$ & 17.65 & 19.32 & 18.64 & 17.03 & 17.29 \\
\hline 12926 & 32.57 & 36.40 & 39.22 & 35.37 & 36.72 \\
\hline $1-4748$ & 32.65 & 35.60 & 36.34 & 33.91 & 33.51 \\
\hline 1 ijis & $2 y .62$ & 32.94 & 31.12 & 36.23 & $35.81)$ \\
\hline $1-5285$ & 19.83 & 22.13 & 20.99 & 23.80 & 27.71 \\
\hline $1-5864$ & 30.40 & 34.77 & 38.09 & 33.62 & 29.67 \\
\hline Average & 21.95 & 24.34 & 25.39 & 24.21 & 24.48 \\
\hline
\end{tabular}
$\mu+\tau_{j}$, for geometry types $1, \mathrm{~S}, \mathrm{R}, \mathrm{F}$, and $\mathrm{C}$ 


\section{APPENDIX B}

A complete listing of all the argon flows and rate data is given in Appendix B. Table B-1 gives the rotameter calibration data used to determine the argon flow versus metering pressure and rotameter float material type.

The argon flow rate data are listed by block number in Table B-2. The description of the column headings is as follows:

OBS $=$ observation number,

HOLE = hole number (see Fig. B-1),

TYPE $=$ hole type,

$\mathrm{X}$ axis $=$ coordinates of hole position in inches (the block center is 0,0 ),

METER = metering pressure (psig), i.e., pressure at rotameter,

Meas 1

Meas 2 = rotameter scale readings,

FLOW $=$ argon flow rate, corrected to standard temperature and pressure, standard liters $/ \mathrm{min}$.

$\mathrm{GAGE}=$ rotameter float material. 
Table B-1. Rotameter calibration data for glass, stainless steel, and tantalum floats

\begin{tabular}{|c|c|c|c|c|}
\hline \multirow{2}{*}{$\begin{array}{l}\text { Rotameter } \\
\text { scale } \\
\text { reading }\end{array}$} & \multirow{2}{*}{$\begin{array}{c}\text { Metering } \\
\text { pressure } \\
\text { (psia) }\end{array}$} & \multicolumn{3}{|c|}{ Argon flow (standard liter/min) } \\
\hline & & Glass & $\begin{array}{c}\text { Stainless } \\
\text { steel }\end{array}$ & Tantalum \\
\hline 2 & 14.7 & 0.22 & 0.75 & 1.39 \\
\hline 3 & 14.7 & 0.49 & 1.33 & 2.27 \\
\hline 4 & 14.7 & 0.83 & 2.05 & 3.30 \\
\hline 5 & 14.7 & 1.22 & 2.78 & 4.38 \\
\hline 6 & 14.7 & 1.58 & 3.53 & 5.42 \\
\hline u & 14.7 & 2.36 & 5.07 & 7.72 \\
\hline 10 & 14.7 & 3.24 & 6.76 & 10.19 \\
\hline 12 & 14.7 & 4.06 & 8.45 & 12.67 \\
\hline 14 & 14.7 & 4.92 & 10.20 & 15.22 \\
\hline 16 & 14.7 & 5.87 & 11.98 & 17.78 \\
\hline 18 & 14.7 & 6.72 & 13.64 & 20.39 \\
\hline 20 & 14.7 & 7.83 & 15.69 & 23.42 \\
\hline 25 & 14.7 & 10.03 & 20.16 & 29.84 \\
\hline 2 & 18.7. & 0.28 & 0.95 & 1.66 \\
\hline 3 & 18.7 & 0.59 & 1.60 & 2.68 \\
\hline 4 & 18.7 & 0.99 & 2.40 & 3.82 \\
\hline 5 & 18.7 & 1.41 & 3.27 & 5.04 \\
\hline 6 & 18.7 & 1.84 & 4.08 & 6.21 \\
\hline 8 & 18.7 & 2.72 & 5.82 & 8.85 \\
\hline 10 & 18.7 & 3.70 & 7.76 & 11.64 \\
\hline 12 & 18.7 & 4.68 & 9.70 & 14.48 \\
\hline 14 & 18.7 & 5.62 & 11.64 & 17.37 \\
\hline 16 & 18.7 & 6.47 & 13.68 & 20.35 \\
\hline 18 & 18.7 & 7.67 & 15.59 & 23.23 \\
\hline 20 & 18.7 & 8.94 & 18.04 & 26.66 \\
\hline 25 & 18.7 & 11.55 & 7.2 .47 & 33.85 \\
\hline 2 & 24.7 & 0.36 & 1.19 & 1.99 \\
\hline 3 & 24.7 & 0.74 & 1.97 & 3.21 \\
\hline 4 & 24.7 & 1.21 & 2.85 & 4.50 \\
\hline 5 & 24.7 & 1.71 & 3.87 & 6.02 \\
\hline 6 & 24.7 & 2.21 & 4.81 & 7.31 \\
\hline 8 & 24.7 & 3.23 & 6.84 & 10.41 \\
\hline 10 & 24.7 & 4.42 & 9.07 & 13.62 \\
\hline 12 & 24.7 & 3.50 & 11.34 & 16.94 \\
\hline 14 & 24.7 & 6.65 & 13.68 & 20.26 \\
\hline 16 & 24.7 & 7.92 & 15.93 & 23.69 \\
\hline 18 & 24.7 & $9 . \dot{0} 2$ & 18.10 & 27.011 \\
\hline 20 & 24.7 & 10.52 & 21.11 & 30.95 \\
\hline 25 & 24.7 & 13.44 & 26.62 & 39.39 \\
\hline
\end{tabular}


Table B-2. Argon permeability data

BLOCK 1-0372 (4 PSIG)

\begin{tabular}{|c|c|c|c|c|c|c|c|c|c|}
\hline OBS & MOLE & TYPE & XAXIS & YAXIS & METER & MEAS 1 & MEAS 2 & FLOW & GAGE \\
\hline $\begin{array}{l}1 \\
2 \\
3 \\
4 \\
5 \\
5 \\
7 \\
8 \\
9 \\
10 \\
11 \\
12 \\
13 \\
14 \\
15 \\
16 \\
17 \\
18 \\
19 \\
20 \\
21 \\
22 \\
23 \\
24 \\
25 \\
26 \\
27 \\
28 \\
29 \\
30 \\
31 \\
32 \\
33 \\
34 \\
35 \\
36 \\
37 \\
38 \\
39 \\
40 \\
41 \\
42 \\
43 \\
44 \\
45 \\
46 \\
47 \\
48 \\
49 \\
50 \\
51 \\
52 \\
53 \\
54 \\
55\end{array}$ & $\begin{array}{r}11 \\
11 \\
154 \\
171 \\
314 \\
324 \\
2 \\
4 \\
5 \\
7 \\
8 \\
110 \\
12 \\
23 \\
37 \\
50 \\
51 \\
65 \\
82 \\
98 \\
99 \\
116 \\
136 \\
153 \\
172 \\
189 \\
209 \\
226 \\
227 \\
243 \\
260 \\
274 \\
275 \\
288 \\
302 \\
313 \\
315 \\
317 \\
318 \\
320 \\
321 \\
323 \\
14 \\
15 \\
17 \\
18 \\
20 \\
21 \\
28 \\
29 \\
31 \\
32 \\
41 \\
43 \\
44\end{array}$ & $\begin{array}{l}C \\
C \\
C \\
C \\
C \\
C \\
F \\
F \\
F \\
F \\
F \\
F \\
F \\
F \\
F \\
F \\
F \\
F \\
F \\
F \\
F \\
F \\
F \\
F \\
F \\
F \\
F \\
F \\
F \\
F \\
F \\
F \\
F \\
F \\
F \\
F \\
F \\
F \\
F \\
F \\
F \\
F \\
R \\
R \\
R \\
R \\
R \\
R \\
R \\
R \\
R \\
R \\
R \\
R \\
R\end{array}$ & $\begin{array}{r}-3.70 \\
3.70 \\
-7.40 \\
7.40 \\
-3.70 \\
3.70 \\
-2.96 \\
-1.48 \\
-0.74 \\
0.74 \\
1.48 \\
2.96 \\
-4.07 \\
4.07 \\
-4.81 \\
4.81 \\
-5.18 \\
5.18 \\
-5.92 \\
5.92 \\
-6.29 \\
6.29 \\
-7.03 \\
7.03 \\
-7.03 \\
7.03 \\
-6.29 \\
6.29 \\
-5.92 \\
5.92 \\
-5.18 \\
5.18 \\
-4.81 \\
4.81 \\
-4.07 \\
4.07 \\
-2.96 \\
-1.48 \\
-0.74 \\
0.74 \\
1.48 \\
2.96 \\
-2.59 \\
-1085 \\
-0.37 \\
0.37 \\
1.85 \\
2.59 \\
-1.48 \\
-0.74 \\
0.74 \\
1.48 \\
-1.85 \\
-0.37 \\
0.37\end{array}$ & $\begin{array}{r}6.41 \\
6.41 \\
0.00 \\
0.00 \\
-6.41 \\
-6.41 \\
6.41 \\
6.41 \\
6.41 \\
6.41 \\
6.41 \\
6.41 \\
5.77 \\
5.77 \\
4.49 \\
4.49 \\
3.85 \\
3.85 \\
2.56 \\
2.56 \\
1.92 \\
1.92 \\
0.64 \\
0.64 \\
-0.64 \\
-0.64 \\
-1.92 \\
-1.92 \\
-2.56 \\
-2.56 \\
-3.85 \\
-3.85 \\
-4.49 \\
-4.49 \\
-5.77 \\
-5.77 \\
-6.41 \\
-6.41 \\
-6.41 \\
-6.41 \\
-6.41 \\
-6.41 \\
5.77 \\
5.77 \\
5.77 \\
5.77 \\
5.77 \\
5.77 \\
5.13 \\
5.13 \\
5.13 \\
5.13 \\
4.49 \\
4.49 \\
4.49\end{array}$ & $\begin{array}{l}4: 1 \\
4: 1 \\
4: 1 \\
4: 1 \\
4: 1 \\
4: 1 \\
4: 1 \\
4: 1 \\
4: 1 \\
4: 1 \\
4: 1 \\
4: 1 \\
4: 1 \\
4: 1 \\
4: 1 \\
4: 1 \\
4: 1 \\
4: 1 \\
4: 1 \\
4: 1 \\
4: 1 \\
4: 1 \\
4: 1 \\
4: 1 \\
4: 1 \\
4: 1 \\
4: 1 \\
4: 1 \\
4: 1 \\
4: 1 \\
4: 1 \\
4: 1 \\
4: 1 \\
4: 1 \\
4: 1 \\
4: 1 \\
4: 1 \\
4: 1 \\
4: 2 \\
4: 1 \\
4: 1 \\
4: 1 \\
4: 1 \\
4: 1 \\
4: 2 \\
4: 2 \\
4: 2 \\
4: 1 \\
4: 2 \\
4: 2 \\
4: 2 \\
4: 2 \\
4: 2 \\
4: 2 \\
4: 2 \\
4 \\
4\end{array}$ & 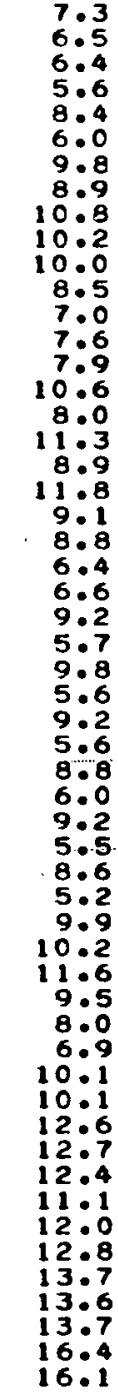 & & $\begin{array}{l}2.4625 \\
2.1047 \\
2.0603 \\
1.7079 \\
2.9622 \\
1.8835 \\
3.6111 \\
3.1923 \\
4.0835 \\
3.7991 \\
3.7050 \\
3.0080 \\
2.3278 \\
2.5979 \\
2.7339 \\
3.9884 \\
2.7794 \\
4.3224 \\
3.1923 \\
4.5632 \\
3.2848 \\
3.1461 \\
2.0603 \\
2.1492 \\
3.3312 \\
1.7517 \\
3.6111 \\
1.7079 \\
3.3312 \\
1.7079 \\
3.1461 \\
1.8835 \\
3.3312 \\
1.6642 \\
3.0540 \\
1.5334 \\
3.6580 \\
3.7991 \\
4.4810 \\
3.4708 \\
2.7794 \\
2.2830 \\
3.7520 \\
3.7520 \\
4.9681 \\
5.0173 \\
4.8701 \\
4.2266 \\
4.6750 \\
5.0665 \\
5.5125 \\
5.4627 \\
5.5125 \\
6.8867 \\
6.7313\end{array}$ & $\begin{array}{l}G L \\
G L \\
G L \\
G L \\
G L \\
G L \\
G L \\
G L \\
G L \\
G L \\
G L \\
G L \\
G L \\
G L \\
G L \\
G L \\
G L \\
G L \\
G L \\
G L \\
G L \\
G L \\
G L \\
G L \\
G L \\
G L \\
G L \\
G L \\
G L \\
G L \\
G L \\
G L \\
G L \\
G L \\
G L \\
G L \\
G L \\
G L \\
G L \\
G L \\
G L \\
G L \\
G L \\
G L \\
G L \\
G L \\
G L \\
G L \\
G L \\
G L \\
G L \\
G L \\
G L \\
G L \\
G L\end{array}$ \\
\hline
\end{tabular}


Table E-2. (continued)

BLOCK 1-0372 (4 PSIG)

\begin{tabular}{|c|c|c|c|c|c|c|c|c|c|}
\hline oBs & HOLE & TrPe & XAXIS & YAXIS & METER & MEASI & MEAS 2 & FLOW & GAGE \\
\hline $\begin{array}{l}56 \\
57 \\
58 \\
59 \\
60 \\
61 \\
62 \\
63 \\
64 \\
65 \\
66 \\
67 \\
68 \\
69 \\
70 \\
71 \\
72 \\
73 \\
74 \\
75 \\
76 \\
77 \\
78 \\
79 \\
80 \\
81 \\
82 \\
83 \\
84 \\
85 \\
86 \\
87 \\
88 \\
89 \\
90 \\
91 \\
92 \\
93 \\
94 \\
95 \\
96 \\
97 \\
98 \\
99 \\
100 \\
101 \\
102 \\
103 \\
104 \\
105 \\
106 \\
107 \\
108 \\
109 \\
110\end{array}$ & 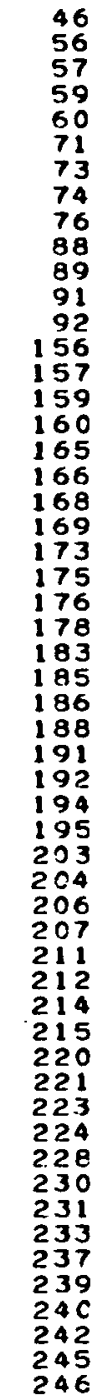 & $\begin{array}{l}R \\
R \\
R \\
R \\
R \\
R \\
R \\
R \\
R \\
R \\
R \\
R \\
R \\
R \\
R \\
R \\
R \\
R \\
R \\
R \\
R \\
R \\
R \\
R \\
R \\
R \\
R \\
R \\
R \\
R \\
R \\
R \\
R \\
R \\
R \\
R \\
R \\
R \\
R \\
R \\
R \\
R \\
R \\
R \\
R \\
R \\
R \\
R \\
R \\
P \\
R \\
R \\
R \\
R \\
R \\
R \\
R \\
R\end{array}$ & $\begin{array}{r}1.85 \\
-1.48 \\
-0.74 \\
0.74 \\
1.48 \\
-1.85 \\
-0.37 \\
0.37 \\
1.85 \\
-1.48 \\
-0.74 \\
0.74 \\
1.48 \\
-5.92 \\
-5.18 \\
-3.70 \\
-2.96 \\
2.96 \\
3.70 \\
5.18 \\
5.92 \\
-6.29 \\
-4.81 \\
-4.077 \\
-2.59 \\
2.59 \\
4.07 \\
4.81 \\
6.29 \\
-5.92 \\
-5.18 \\
-3.70 \\
-2.996 \\
2.96 \\
3.70 \\
5.18 \\
5.92 \\
-4.81 \\
-4.07 \\
-2.59 \\
-1.85 \\
1.85 \\
2.59 \\
4.007 \\
4.81 \\
-5.18 \\
-3.70 \\
-2.96 \\
-1.48 \\
1.48 \\
2.996 \\
3.70 \\
5.18 \\
-4.81 \\
-4.07\end{array}$ & 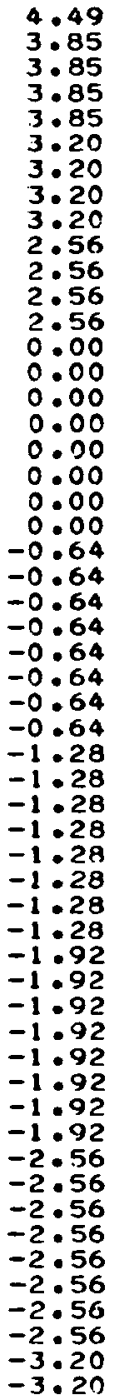 & $\begin{array}{l}4 \cdot 2 \\
4: 2 \\
4: 2 \\
4: 2 \\
4: 2 \\
4: 2 \\
4: 2 \\
4: 22 \\
4: 3 \\
4: 2 \\
4: 2 \\
4: 3 \\
4: 3 \\
4: 1 \\
4: 1 \\
4: 2 \\
4: 2 \\
4: 3 \\
4: 2 \\
4: 2 \\
4: 2 \\
4: 1 \\
4: 1 \\
4: 2 \\
4: 3 \\
4: 3 \\
4: 2 \\
4: 2 \\
4: 1 \\
4: 1 \\
4: 1 \\
4: 3 \\
4: 2 \\
4: 3 \\
4: 22 \\
4: 2 \\
4: 1 \\
4: 1 \\
4: 2 \\
4: 2 \\
4: 3 \\
4: 3 \\
4: 3 \\
4: 2 \\
4: 2 \\
4: 1 \\
4: 2 \\
4: 2 \\
4: 3 \\
4: 3 \\
4: 2 \\
4: 2 \\
4: 1 \\
4: 1 \\
4: 2\end{array}$ & $\begin{array}{l}16.6 \\
15: 8 \\
17.2 \\
19.4 \\
19.0 \\
17.1 \\
20.9 \\
211.1 \\
23.3 \\
20.6 \\
20.7 \\
23.6 \\
22.2 \\
9.0 \\
9.5 \\
17.1 \\
17.8 \\
14.8 \\
21.7 \\
14.8 \\
11.0 \\
10.06 \\
12.7 \\
17.8 \\
23.3 \\
14.4 \\
20.6 \\
15.5 \\
8.4 \\
10.7 \\
11.4 \\
21.2 \\
22.3 \\
24.1 \\
19.6 \\
11.4 \\
18.2 \\
13.0 \\
17.6 \\
22.7 \\
23.7 \\
15.2 \\
23.0 \\
16.8 \\
11.6 \\
10.9 \\
18.9 \\
21.4 \\
24.1 \\
14.0 \\
21.4 \\
16.5 \\
.8 .0 \\
11.3 \\
13.4\end{array}$ & & $\begin{array}{r}6.9906 \\
6.5767 \\
7.3042 \\
8.4768 \\
8.2609 \\
7.2518 \\
9.2968 \\
9.4074 \\
10.6745 \\
9.1315 \\
9.1865 \\
10.8463 \\
10.0504 \\
3.2375 \\
3.4708 \\
7.2518 \\
7.6205 \\
12.4603 \\
9.7410 \\
6.0659 \\
4.1923 \\
3.9884 \\
5.0014 \\
7.6205 \\
10.6745 \\
12.0646 \\
9.1315 \\
6.4226 \\
2.9622 \\
4.0359 \\
4.3704 \\
9.4908 \\
10.0772 \\
11.1340 \\
8.5852 \\
4.3845 \\
2.8707 \\
5.1487 \\
705147 \\
10.3029 \\
10.9037 \\
12.8580 \\
10.5034 \\
7.0949 \\
4.4810 \\
4.1311 \\
8.2072 \\
9.5739 \\
11.1340 \\
11.6708 \\
9.5739 \\
6.9386 \\
2.7794 \\
4.3224 \\
5.3632\end{array}$ & $\begin{array}{l}G L \\
G L \\
G L \\
G L \\
G L \\
G L \\
G L \\
G L \\
G L \\
G L \\
G L \\
G L \\
G L \\
G L \\
G L \\
G L \\
G L \\
S S \\
G L \\
G L \\
G L \\
G L \\
G L \\
G L \\
G L \\
S S \\
G L \\
G L \\
G L \\
G L \\
G L \\
G L \\
G L \\
G L \\
G L \\
G L \\
G L \\
G L \\
G L \\
G L \\
G L \\
S S \\
G L \\
G L \\
G L \\
G L \\
G L \\
G L \\
G L \\
S S \\
G L \\
G L \\
G L \\
G L \\
G L \\
\end{array}$ \\
\hline
\end{tabular}


Table B-2. (continued)

RLOCK 1-0372 (4 PSIG)

\begin{tabular}{|c|c|c|c|c|c|c|c|c|c|}
\hline OES & HOLE & TYPE & $\times A \times I S$ & YAXIS & ME TER & MEAS 1 & MEAS 2 & FLOW & GAGE \\
\hline 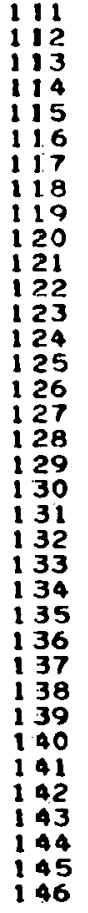 & $\begin{array}{l}248 \\
249 \\
254 \\
255 \\
257 \\
258 \\
262 \\
263 \\
271 \\
272 \\
276 \\
278 \\
285 \\
287 \\
290 \\
291 \\
299 \\
300 \\
107 \\
108 \\
124 \\
128 \\
143 \\
146 \\
179 \\
182 \\
197 \\
201 \\
217 \\
218 \\
12.5 \\
127 \\
162 \\
163 \\
198 \\
200\end{array}$ & $\begin{array}{l}R \\
R \\
R \\
R \\
R \\
R \\
R \\
R \\
R \\
R \\
R \\
R \\
R \\
R \\
R \\
R \\
R \\
R \\
R \\
R \\
S \\
S \\
S \\
S \\
S \\
S \\
S \\
S \\
S \\
S \\
S \\
S \\
I \\
I \\
I \\
Z \\
I \\
I\end{array}$ & $\begin{array}{r}-2.59 \\
-1.35 \\
1.85 \\
2.59 \\
4.07 \\
4.81 \\
-3.70 \\
-2.96 \\
2: .96 \\
3: 75 \\
-4.07 \\
-2.59 \\
2.59 \\
4.07 \\
-3.70 \\
-2.96 \\
2.96 \\
3.70 \\
-0.37 \\
0.37 \\
-1: 48 \\
1.48 \\
-1.85 \\
1.85 \\
-1.85 \\
1.855 \\
-1.48 \\
1.48 \\
-0.37 \\
0.37 \\
-0.74 \\
0.74 \\
-1.48 \\
1.488 \\
-0.74 \\
0.74\end{array}$ & $\begin{array}{r}-3.20 \\
-3.20 \\
-3.20 \\
-3.20 \\
-3.20 \\
-3.20 \\
-3.85 \\
-3.85 \\
-3.85 \\
-3.85 \\
-4.49 \\
-4.49 \\
-4.49 \\
-4.86 \\
-5.13 \\
-5.13 \\
-5.13 \\
-5.13 \\
1.92 \\
1.92 \\
1.28 \\
1.28 \\
0.64 \\
0.64 \\
-0.64 \\
-0.64 \\
-1.28 \\
-1.28 \\
-1.92 \\
-1.92 \\
1.28 \\
1.28 \\
0.00 \\
0.00 \\
-1.28 \\
-1.28\end{array}$ & $\begin{array}{l}4 \cdot 3 \\
4: 3 \\
4: 3 \\
4: 2 \\
4: 2 \\
4: 1 \\
4: 2 \\
4: 2 \\
4: 2 \\
4: 2 \\
4: 1 \\
4: 2 \\
4: 2 \\
4: 1 \\
4: 1 \\
4: 1 \\
4: 2 \\
4: 1 \\
4: 3 \\
4: 2 \\
4: 2 \\
4: 2 \\
4: 2 \\
4: 3 \\
4: 3 \\
4: 3 \\
4: 3 \\
4: 3 \\
4: 3 \\
4: 3 \\
4: 2 \\
4: 2 \\
4: 2 \\
4: 2 \\
4: 3 \\
4: 3\end{array}$ & $\begin{array}{l}23.0 \\
23.9 \\
23.9 \\
19.9 \\
12.0 \\
8.0 \\
13.5 \\
17.3 \\
15.6 \\
110.0 \\
10.5 \\
15.5 \\
13.7 \\
7.08 \\
10.5 \\
11.5 \\
9.5 \\
7.2 \\
13.6 \\
10.6 \\
111.7 \\
11.1 \\
11.5 \\
13.0 \\
13.1 \\
15.2 \\
13.2 \\
14.8 \\
14.5 \\
13.9 \\
9.5 \\
10.6 \\
10.4 \\
111.2 \\
12.4 \\
14.2\end{array}$ & & $\begin{array}{r}10.5034 \\
11.0187 \\
11.0187 \\
8.7483 \\
4.6750 \\
2.7794 \\
5.4129 \\
7.3567 \\
6.4739 \\
4.1923 \\
3.9410 \\
6.4226 \\
5.5125 \\
2.5885 \\
3.9410 \\
4.4185 \\
3.4822 \\
2.41 .75 \\
11.2789 \\
8.3753 \\
9.4150 \\
8.8461 \\
9.2249 \\
10.6947 \\
10.7918 \\
12.8580 \\
10.8890 \\
12.4603 \\
12.1633 \\
11.5726 \\
7.3500 \\
8.3753 \\
8.1878 \\
8.9406 \\
10.1148 \\
11.8675\end{array}$ & $\begin{array}{l}G L \\
G L \\
G L \\
G L \\
G L \\
G L \\
G L \\
G L \\
G L \\
G L \\
G L \\
G L \\
G L \\
G L \\
G L \\
G L \\
G L \\
G L \\
S S \\
S S \\
S S \\
S S \\
\text { SS } \\
\text { SS } \\
\text { SS } \\
\text { SS } \\
\text { SS } \\
\text { SS } \\
\text { SS } \\
\text { SS } \\
\text { SS } \\
\text { SS } \\
\text { SS } \\
\text { SS } \\
\text { SS } \\
\text { SS }\end{array}$ \\
\hline
\end{tabular}


Table B-2. (continued)

BLOCK 1-0637 (4 PSIG)

\begin{tabular}{|c|c|c|c|c|c|c|c|c|c|}
\hline OBS & HOLE & TYPE & XAXIS & YAXIS & ME TER & MEASI & MEAS 2 & FLOM & GAGE \\
\hline $\begin{array}{l}1 \\
2 \\
3 \\
4 \\
5 \\
6 \\
7 \\
8 \\
9 \\
10 \\
11 \\
1.2 \\
13 \\
14 \\
15 \\
16 \\
17 \\
18 \\
19 \\
20 \\
21 \\
22 \\
23 \\
24 \\
25 \\
26 \\
27 \\
28 \\
29 \\
30 \\
31 \\
32 \\
33 \\
34 \\
35 \\
36 \\
37 \\
38 \\
39 \\
40 \\
41 \\
42 \\
43 \\
44 \\
45 \\
46 \\
47 \\
48 \\
49 \\
50 \\
51 \\
52 \\
53 \\
54 \\
55\end{array}$ & 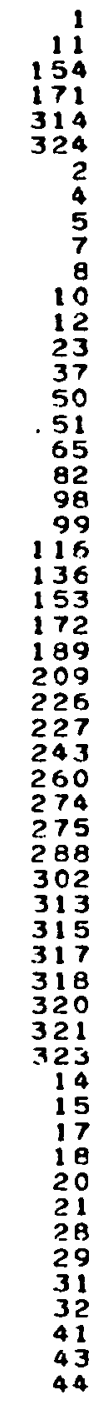 & $\begin{array}{l}C \\
C \\
C \\
C \\
C \\
C \\
F \\
F \\
F \\
F \\
F \\
F \\
F \\
F \\
F \\
F \\
F \\
F \\
F \\
F \\
F \\
F \\
F \\
F \\
F \\
F \\
F \\
F \\
F \\
F \\
F \\
F \\
F \\
F \\
F \\
F \\
F \\
F \\
F \\
F \\
F \\
F \\
R \\
R \\
R \\
R \\
R \\
R \\
R \\
R \\
R \\
R \\
R \\
R \\
R\end{array}$ & $\begin{array}{r}-3.70 \\
3.70 \\
-7.40 \\
7.40 \\
-3.770 \\
3.70 \\
-2.96 \\
-1.48 \\
-0.74 \\
0: 74 \\
1.48 \\
2.96 \\
-4.07 \\
4.07 \\
-4.81 \\
4.81 \\
-5.18 \\
5.18 \\
-5.92 \\
5.92 \\
-6.29 \\
6.29 \\
-7.03 \\
7.03 \\
-7.03 \\
7.03 \\
-6.29 \\
5: 29 \\
-5.92 \\
5.92 \\
-5.18 \\
5.18 \\
-4.81 \\
4: 81 \\
-4.097 \\
4.07 \\
-2.96 \\
-1.48 \\
-0.74 \\
0.74 \\
1.48 \\
2.96 \\
-2.59 \\
-1.85 \\
-0.37 \\
0.37 \\
1.85 \\
2.59 \\
-1.48 \\
-0.74 \\
0.74 \\
1.48 \\
-1.85 \\
-0.37 \\
0.37\end{array}$ & $\begin{array}{r}6.41 \\
6.41 \\
0.00 \\
0.00 \\
-6.41 \\
-6.41 \\
6.41 \\
6.41 \\
6.41 \\
6.41 \\
6.41 \\
6.41 \\
5.77 \\
5.77 \\
4.49 \\
4.49 \\
3.85 \\
3.85 \\
2.56 \\
2.56 \\
1.92 \\
1.92 \\
0.64 \\
0.64 \\
-0.64 \\
-0.64 \\
-1.92 \\
-1.92 \\
-2.56 \\
-2.56 \\
-3.85 \\
-3.85 \\
-4.49 \\
-4.49 \\
-5.77 \\
-5.77 \\
-6.41 \\
-6.41 \\
-6.41 \\
-6.41 \\
-6.41 \\
-6.41 \\
5.77 \\
5.77 \\
5.77 \\
5.77 \\
5.77 \\
5077 \\
5013 \\
5.13 \\
5013 \\
5.13 \\
4.49 \\
4.49 \\
4.49\end{array}$ & $\begin{array}{l}4 \cdot 2 \\
4: 1 \\
4: 2 \\
4: 2 \\
4: 1 \\
4: 2 \\
4: 2 \\
4: 2 \\
4: 2 \\
4: 2 \\
4: 2 \\
4: 2 \\
4: 2 \\
4: 2 \\
4: 2 \\
4: 2 \\
4: 2 \\
4: 2 \\
4: 2 \\
4: 2 \\
4: 2 \\
4: 2 \\
4: 2 \\
4: 2 \\
4: 2 \\
4: 2 \\
4: 2 \\
4: 2 \\
4: 2 \\
4: 2 \\
4: 2 \\
4: 2 \\
4: 2 \\
4: 22 \\
4: 2 \\
4: 2 \\
4: 2 \\
4: 2 \\
4: 2 \\
4: 2 \\
4: 2 \\
4: 2 \\
4: 2 \\
4: 2 \\
4: 22 \\
4: 2 \\
4: 2 \\
4: 2 \\
4: 3 \\
4: 3 \\
4: 3 \\
4: 3 \\
4: 3 \\
4: 3 \\
4: 4 \\
2\end{array}$ & $\begin{array}{l}11.0 \\
10.7 \\
111.6 \\
1506 \\
10.7 \\
16.4 \\
14.0 \\
16.7 \\
16.6 \\
16.5 \\
15.6 \\
12.9 \\
12.5 \\
12.7 \\
16.3 \\
17.6 \\
15.6 \\
18.7 \\
15.4 \\
12.0 \\
13.9 \\
11.7 \\
12.3 \\
11.0 \\
13.2 \\
111.0 \\
17.2 \\
12.6 \\
18.3 \\
12.9 \\
17.0 \\
12.8 \\
14.8 \\
13.0 \\
12.7 \\
100.4 \\
12.7 \\
16.4 \\
10.4 \\
111.5 \\
10.9 \\
10.6 \\
11.0 \\
13.0 \\
12.5 \\
12.9 \\
110.8 \\
10.6 \\
16.1 \\
16.1 \\
15.9 \\
14.8 \\
18.9 \\
18.6 \\
19.1\end{array}$ & & $\begin{array}{r}4.1923 \\
4.0359 \\
4.4810 \\
6.4739 \\
6.0359 \\
6.8867 \\
5.6625 \\
7.0427 \\
6.9906 \\
6.9386 \\
6.4739 \\
5.1157 \\
1.9191 \\
5.0173 \\
6.8348 \\
1.5147 \\
6.4739 \\
8.0998 \\
6.3715 \\
9.7011 \\
5.6125 \\
9.4150 \\
4.8212 \\
8.7517 \\
5.2640 \\
8.7517 \\
1.3042 \\
10.2765 \\
7.8560 \\
10.5658 \\
1.1994 \\
10.4693 \\
6.0659 \\
10.6625 \\
5.01173 \\
8.1878 \\
5.0173 \\
6.8867 \\
8.1878 \\
9.2249 \\
8.6574 \\
8.3753 \\
8.7517 \\
10.6625 \\
10.1803 \\
10.5658 \\
9.5102 \\
8.3753 \\
13.7597 \\
13.7597 \\
13.5584 \\
12.4603 \\
16.6271 \\
16.3154 \\
16.8841\end{array}$ & $\begin{array}{l}G L \\
G L \\
G L \\
G L \\
G L \\
G L \\
G L \\
G L \\
G L \\
G L \\
G L \\
G L \\
G L \\
G L \\
G L \\
G L \\
G L \\
G L \\
G L \\
S S \\
G L \\
S S \\
G L \\
S S \\
G L \\
S S \\
G L \\
S S \\
G L \\
S S \\
G L \\
S S \\
G L \\
S S \\
G L \\
S S \\
G L \\
G L \\
S S \\
S S \\
S S \\
S S \\
S S \\
S S \\
S S \\
S S \\
S S \\
S S \\
S S \\
S S \\
S S \\
S S \\
S S \\
S S \\
S S\end{array}$ \\
\hline
\end{tabular}


Table B-2. (continued)

BLOCK 1-0637 (4 PSIG)

\begin{tabular}{|c|c|c|c|c|c|c|c|c|c|}
\hline OBS & HOLE & TYPE & $X A \times 15$ & YAXIS & METER & MEAS 1 & MEAS 2 & FLON & GAGE \\
\hline $\begin{array}{l}56 \\
57 \\
58 \\
59 \\
60 \\
61 \\
62 \\
63 \\
64 \\
65 \\
56 \\
67 \\
68 \\
69 \\
70 \\
71 \\
72 \\
73 \\
74 \\
75 \\
76 \\
77 \\
78 \\
79 \\
80 \\
81 \\
82 \\
83 \\
84 \\
85 \\
86 \\
87 \\
88 \\
89 \\
90 \\
91 \\
92 \\
93 \\
94 \\
95 \\
96 \\
97 \\
98 \\
099 \\
100 \\
101 \\
102 \\
103 \\
104 \\
105 \\
106 \\
107 \\
108 \\
109 \\
110\end{array}$ & $\begin{array}{l}46 \\
56 \\
57 \\
59 \\
50 \\
71 \\
73 \\
74 \\
76 \\
88 \\
89 \\
81 \\
91 \\
92 \\
156 \\
157 \\
159 \\
160 \\
165 \\
166 \\
168 \\
169 \\
173 \\
175 \\
176 \\
178 \\
173 \\
185 \\
186 \\
188 \\
191 \\
192 \\
194 \\
195 \\
203 \\
204 \\
206 \\
207 \\
211 \\
212 \\
214 \\
215 \\
220 \\
221 \\
223 \\
224 \\
228 \\
230 \\
231 \\
233 \\
237 \\
239 \\
240 \\
242 \\
245 \\
246\end{array}$ & $\begin{array}{l}R \\
R \\
R \\
R \\
R \\
R \\
R \\
R \\
R \\
R \\
R \\
R \\
R \\
R \\
R \\
R \\
R \\
R \\
R \\
R \\
R \\
R \\
R \\
R \\
R \\
R \\
R \\
R \\
R \\
R \\
R \\
R \\
R \\
R \\
R \\
R \\
R \\
R \\
R \\
R \\
R \\
R \\
R \\
R \\
R \\
R \\
R \\
R \\
R \\
R \\
R \\
R \\
R \\
R \\
R \\
R \\
R \\
R \\
R \\
R \\
R \\
R\end{array}$ & $\begin{array}{r}1.85 \\
-1: 48 \\
-6: 74 \\
0: 74 \\
1: 48 \\
-1.85 \\
-0.37 \\
0: 37 \\
1.85 \\
-1.48 \\
-0.74 \\
0.74 \\
1.48 \\
-5.92 \\
-5.18 \\
-3.70 \\
-2.96 \\
2.96 \\
3.70 \\
5.18 \\
5.92 \\
-6.29 \\
-4.81 \\
-4.07 \\
-2.59 \\
2.59 \\
4.07 \\
4.81 \\
6.29 \\
-5.92 \\
-5.18 \\
-3.70 \\
-2.96 \\
2.96 \\
3.70 \\
5.18 \\
5.92 \\
-4.81 \\
-4.07 \\
-2.59 \\
-1.85 \\
1.85 \\
2.59 \\
4.07 \\
4.81 \\
-5.18 \\
-3.70 \\
-2.96 \\
-1.48 \\
11.48 \\
2.96 \\
3.70 \\
5.18 \\
-4.81 \\
-4.07\end{array}$ & 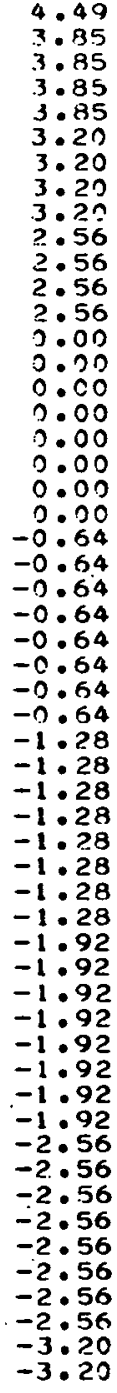 & $\begin{array}{l}4: 3 \\
4: 4 \\
4: 4 \\
4: 4 \\
4: 4 \\
4: 5 \\
4: 5 \\
4: 5 \\
4: 5 \\
4: 6 \\
4: 5 \\
4: 5 \\
4: 5 \\
4: 3 \\
4: 3 \\
4: 5 \\
4: 5 \\
4: 5 \\
4: 4 \\
4: 4 \\
4: 3 \\
4: 2 \\
4: 4 \\
4: 5 \\
4: 5 \\
4: 5 \\
4: 44 \\
4: 4 \\
4: 3 \\
4: 3 \\
4: 3 \\
4: 5 \\
4: 4 \\
4: 5 \\
4: 4 \\
4: 4 \\
4: 3 \\
4: 4 \\
4: 4 \\
4: 4 \\
4: 6 \\
4: 5 \\
4: 5 \\
4: 4 \\
4: 4 \\
4: 3 \\
4: 4 \\
4: 5 \\
4: 5 \\
4: 5 \\
4: 5 \\
4: 4 \\
4: 3 \\
4: 2 \\
4: 3\end{array}$ & 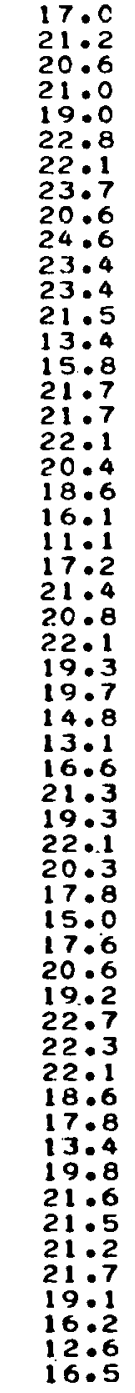 & & 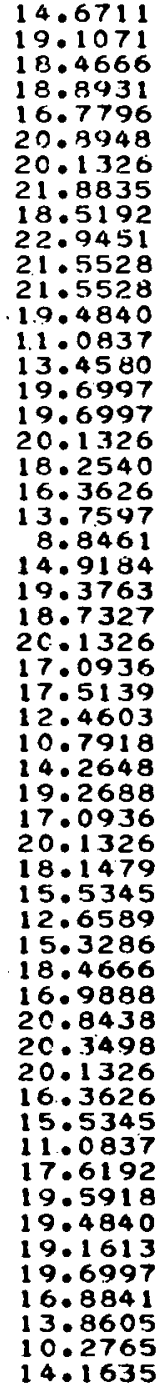 & 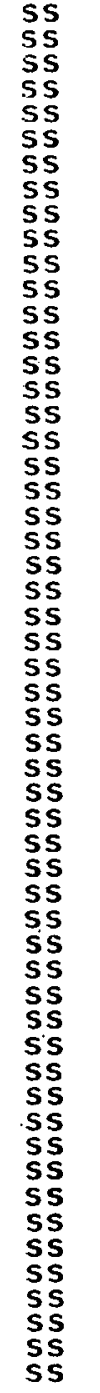 \\
\hline
\end{tabular}


Table B-2. (continued)

BLOCK $1-0637^{\circ}$ (4 PSIG)

\begin{tabular}{|c|c|c|c|c|c|c|c|c|c|}
\hline OBS & HOLE & TYPE & XAXIS & YAXIS & METER & MEASI & MEAS 2 & FLOW & GAGE \\
\hline $\begin{array}{lll}1 & 1 & 1 \\
1 & 1 & 2 \\
1 & 1 & 3 \\
1 & 1 & 4 \\
1 & 1 & 5 \\
1 & 1 & 6 \\
1 & 1 & 7 \\
1 & 1 & 8 \\
1 & 1 & 9 \\
1 & 20 \\
1 & 21 \\
1 & 22 \\
1 & 23 \\
1 & 24 \\
1 & 25 \\
1 & 26 \\
1 & 27 \\
1 & 28 \\
1 & 29 \\
1 & 30 \\
1 & 31 \\
1 & 32 \\
1 & 33 \\
1 & 34 \\
1 & 35 \\
1 & 36 \\
1 & 37 \\
1 & 38 \\
1 & 39 \\
1 & 40 \\
1 & 41 \\
1 & 42 \\
1 & 43 \\
1 & 44 \\
1 & 45 \\
1 & 46\end{array}$ & $\begin{array}{l}248 \\
249 \\
254 \\
255 \\
257 \\
258 \\
262 \\
263 \\
271 \\
272 \\
276 \\
278 \\
285 \\
287 \\
290 \\
291 \\
299 \\
300 \\
107 \\
109 \\
124 \\
128 \\
143 \\
146 \\
179 \\
182 \\
197 \\
201 \\
217 \\
218 \\
1125 \\
127 \\
162 \\
163 \\
198 \\
200\end{array}$ & $\begin{array}{l}R \\
R \\
R \\
R \\
R \\
R \\
R \\
R \\
R \\
R \\
R \\
R \\
R \\
R \\
R \\
R \\
R \\
R \\
P \\
S \\
S \\
S \\
S \\
S \\
S \\
S \\
S \\
S \\
S \\
S \\
S \\
I \\
I \\
I \\
I \\
I \\
I\end{array}$ & $\begin{array}{r}-2.59 \\
-1.85 \\
1.85 \\
2.59 \\
4.07 \\
4.81 \\
-3.70 \\
-2.96 \\
2.96 \\
3.70 \\
-4.07 \\
-2.59 \\
2.59 \\
4.07 \\
-3.70 \\
-2.96 \\
2.96 \\
3.70 \\
-0.37 \\
0.37 \\
-1.48 \\
1.48 \\
-1.85 \\
1.85 \\
-1.85 \\
1.85 \\
-1.49 \\
1.48 \\
-3.37 \\
0.37 \\
-0.74 \\
0.74 \\
-1.48 \\
1.48 \\
-0.74 \\
0.74\end{array}$ & $\begin{array}{r}-3.20 \\
-3.20 \\
-3.20 \\
-3.20 \\
-3.20 \\
-3.20 \\
-3.85 \\
-3.85 \\
-3.85 \\
-3.85 \\
-4.49 \\
-4.49 \\
-4.49 \\
-4.86 \\
-5.13 \\
-5.13 \\
-5.13 \\
-5.13 \\
1.92 \\
1: 92 \\
1.28 \\
1.28 \\
0.64 \\
0.64 \\
-0.64 \\
-0.64 \\
-1.28 \\
-1.28 \\
-1.92 \\
-1.92 \\
1.28 \\
1.28 \\
0.00 \\
0.00 \\
-1.28 \\
-1.28\end{array}$ & $\begin{array}{l}4: 4 \\
4: 5 \\
4: 4 \\
4: 5 \\
4: 4 \\
4: 4 \\
4: 3 \\
4: 5 \\
4: 4 \\
4: 4 \\
4: 2 \\
4: 3 \\
4: 4 \\
4: 3 \\
4: 2 \\
4: 2 \\
4: 3 \\
4: 3 \\
4: 6 \\
4: 6 \\
4: 6 \\
4: 6 \\
4: 6 \\
4: 6 \\
4: 6 \\
4: 5 \\
4: 6 \\
4: 6 \\
4: 6 \\
4: 6 \\
4: 6 \\
4: 6 \\
4: 5 \\
4: 6 \\
4: 6 \\
4.6\end{array}$ & $\begin{array}{l}19.0 \\
21: 3 \\
20: 5 \\
21: 6 \\
17.2 \\
17.0 \\
15: 8 \\
20: 3 \\
19.8 \\
17.7 \\
12.3 \\
15.2 \\
18.7 \\
15.7 \\
10: 5 \\
11.7 \\
15.6 \\
14.8 \\
23.2 \\
24.5 \\
25.0 \\
23.9 \\
24.3 \\
24.1 \\
23.4 \\
22.1 \\
23.8 \\
22.9 \\
23.9 \\
24.6 \\
23.2 \\
22.9 \\
22.1 \\
22.4 \\
23.3 \\
23.5\end{array}$ & & $\begin{array}{r}16.7796 \\
19.2688 \\
18.3602 \\
19.5918 \\
14.9184 \\
14.7140 \\
13.4580 \\
18.1997 \\
17.6192 \\
15.4315 \\
9.9882 \\
12.8580 \\
16.4667 \\
13.3577 \\
8.2814 \\
9.4150 \\
13.2575 \\
12.4603 \\
21.3926 \\
22.8334 \\
23.3930 \\
22.1659 \\
22.6104 \\
22.3879 \\
21.6129 \\
20.1326 \\
22.0551 \\
21.0629 \\
22.1659 \\
22.9451 \\
21.3926 \\
21.0629 \\
20.1326 \\
20.5160 \\
2115027 \\
21.7233\end{array}$ & 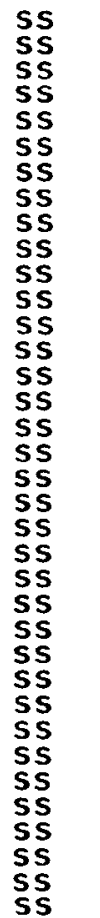 \\
\hline
\end{tabular}


Table B-2. (continued)

FLOCK $1-1046$ (4 DSIG)

\begin{tabular}{|c|c|c|c|c|c|c|c|c|c|}
\hline OAS & HOLE & TYPE & $X A \times I S$ & YAXIS & METER & MEASI & MEAS 2 & FLOW & GAGE \\
\hline $\begin{array}{l}1 \\
2 \\
3 \\
4 \\
5 \\
6 \\
7 \\
8 \\
9 \\
10 \\
11 \\
12 \\
13 \\
14 \\
15 \\
16 \\
17 \\
18 \\
19 \\
20 \\
21 \\
22 \\
23 \\
24 \\
25 \\
26 \\
27 \\
28 \\
29 \\
30 \\
31 \\
32 \\
33 \\
34 \\
35 \\
36 \\
37 \\
38 \\
39 \\
40 \\
41 \\
42 \\
43 \\
44 \\
45 \\
46 \\
47 \\
48 \\
499 \\
50 \\
51 \\
52 \\
53 \\
54 \\
55\end{array}$ & $\begin{array}{r}11 \\
11 \\
154 \\
171 \\
314 \\
324 \\
2 \\
4 \\
5 \\
7 \\
9 \\
8 \\
110 \\
12 \\
23 \\
37 \\
50 \\
51 \\
65 \\
82 \\
98 \\
99 \\
116 \\
136 \\
153 \\
172 \\
189 \\
239 \\
226 \\
227 \\
243 \\
260 \\
274 \\
275 \\
288 \\
302 \\
313 \\
315 \\
317 \\
318 \\
320 \\
321 \\
323 \\
14 \\
15 \\
17 \\
118 \\
20 \\
21 \\
28 \\
29 \\
31 \\
32 \\
41 \\
43 \\
44\end{array}$ & $\begin{array}{l}C \\
C \\
C \\
C \\
C \\
C \\
F \\
F \\
F \\
F \\
F \\
F \\
F \\
F \\
F \\
F \\
F \\
F \\
F \\
F \\
F \\
F \\
F \\
F \\
F \\
F \\
F \\
F \\
F \\
F \\
F \\
F \\
F \\
F \\
F \\
F \\
F \\
F \\
F \\
F \\
F \\
F \\
R \\
R \\
R \\
R \\
R \\
R \\
R \\
R \\
R \\
R \\
R \\
R \\
R\end{array}$ & $\begin{array}{r}-3.70 \\
3.70 \\
-7.40 \\
7.40 \\
-3.70 \\
3.70 \\
-2.96 \\
-1.48 \\
-0.74 \\
0.74 \\
1.48 \\
2.96 \\
-4.07 \\
4.07 \\
-4.81 \\
4.81 \\
-5.18 \\
5.18 \\
-5.92 \\
5.92 \\
-6.29 \\
6.29 \\
-7.03 \\
7.03 \\
-7.03 \\
7.03 \\
-6.29 \\
6.29 \\
-5.92 \\
5.92 \\
-5.18 \\
5.18 \\
-4.81 \\
4.81 \\
-4.07 \\
4.07 \\
-2.96 \\
-1.48 \\
-0.74 \\
0.74 \\
1.48 \\
2.96 \\
-2.59 \\
-1.85 \\
-0.37 \\
0.37 \\
1.85 \\
2.59 \\
-1.49 \\
-0.74 \\
0.74 \\
11.48 \\
-1.85 \\
-0.37 \\
0.37\end{array}$ & $\begin{array}{r}6.41 \\
6.41 \\
0.009 \\
0.00 \\
-6.41 \\
-6.41 \\
6.41 \\
6.41 \\
6.41 \\
6.41 \\
6.41 \\
6.41 \\
5.77 \\
5.77 \\
4.49 \\
4.49 \\
3.85 \\
3.85 \\
2.56 \\
2.56 \\
1.92 \\
1.92 \\
0.64 \\
0.64 \\
-0.64 \\
-0.64 \\
-1.92 \\
-1.92 \\
-2.56 \\
-2.56 \\
-3.85 \\
-3.85 \\
-4.49 \\
-4.49 \\
-5.77 \\
-5.77 \\
-6.41 \\
-6.41 \\
-6.41 \\
-6.41 \\
-6.41 \\
-6.41 \\
5.77 \\
5.77 \\
5.77 \\
5.77 \\
5.77 \\
5.77 \\
5.13 \\
5.13 \\
5.13 \\
5.13 \\
4.49 \\
4.49 \\
4.49\end{array}$ & $\begin{array}{l}4: 1 \\
4: 1 \\
4: 1 \\
4: 1 \\
4: 1 \\
4: 1 \\
4: 1 \\
4: 1 \\
4: 1 \\
4: 1 \\
4: 1 \\
4: 1 \\
4: 1 \\
4: 1 \\
4: 1 \\
4: 1 \\
4: 1 \\
4: 1 \\
4: 1 \\
4: 1 \\
4: 1 \\
4: 2 \\
4: 1 \\
4: 1 \\
4: 1 \\
4: 2 \\
4: 1 \\
4: 2 \\
4: 1 \\
4: 2 \\
4: 1 \\
4: 2 \\
4: 1 \\
4: 2 \\
4: 1 \\
4: 1 \\
4: 1 \\
4: 1 \\
4: 1 \\
4: 2 \\
4: 2 \\
4: 1 \\
4: 1 \\
4: 1 \\
4: 1 \\
4: 1 \\
4: 1 \\
4: 1 \\
4: 1 \\
4: 2 \\
4: 2 \\
4: 2 \\
4: 2 \\
4: 2 \\
4: 2 \\
4 \\
4\end{array}$ & 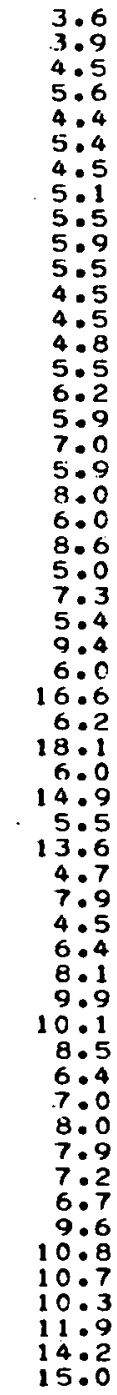 & & $\begin{array}{l}\text { C.8475 } \\
0.9747 \\
1.2310 \\
1.7079 \\
1.1881 \\
1.6205 \\
1.2310 \\
1.4900 \\
1.6642 \\
1.8395 \\
1.6642 \\
1.2310 \\
1.2310 \\
1.3602 \\
1.6642 \\
1.9718 \\
1.8395 \\
2.3278 \\
1.8395 \\
2.7794 \\
1.8835 \\
3.0641 \\
1.4467 \\
2.4625 \\
1.6205 \\
3.4354 \\
1.8835 \\
6.9906 \\
1.9718 \\
7.7796 \\
1.8835 \\
6.1166 \\
1.6642 \\
5.4627 \\
1.3170 \\
2.7339 \\
1.2310 \\
2.0603 \\
2.8250 \\
3.6699 \\
3.7642 \\
3.0080 \\
2.0603 \\
2.3278 \\
2.7794 \\
2.7339 \\
2.4175 \\
2.1937 \\
3.5175 \\
4.0967 \\
4.0489 \\
3.8588 \\
4.6264 \\
5.7629 \\
6.1674\end{array}$ & $\begin{array}{l}G L \\
G L \\
G L \\
G L \\
G L \\
G L \\
G L \\
G L \\
G L \\
G L \\
G L \\
G L \\
G L \\
G L \\
G L \\
G L \\
G L \\
G L \\
G L \\
G L \\
G L \\
G L \\
G L \\
G L \\
G L \\
G L \\
G L \\
G L \\
G L \\
G L \\
G L \\
G L \\
G L \\
G L \\
G L \\
G L \\
G L \\
G L \\
G L \\
G L \\
G L \\
G L \\
G L \\
G L \\
G L \\
G L \\
G L \\
G L \\
G L \\
G L \\
G L \\
G L \\
G L \\
G L \\
G L \\
G\end{array}$ \\
\hline
\end{tabular}


Table B-2. (continued)

3LOCK $1-1046$ (4 PSIG)

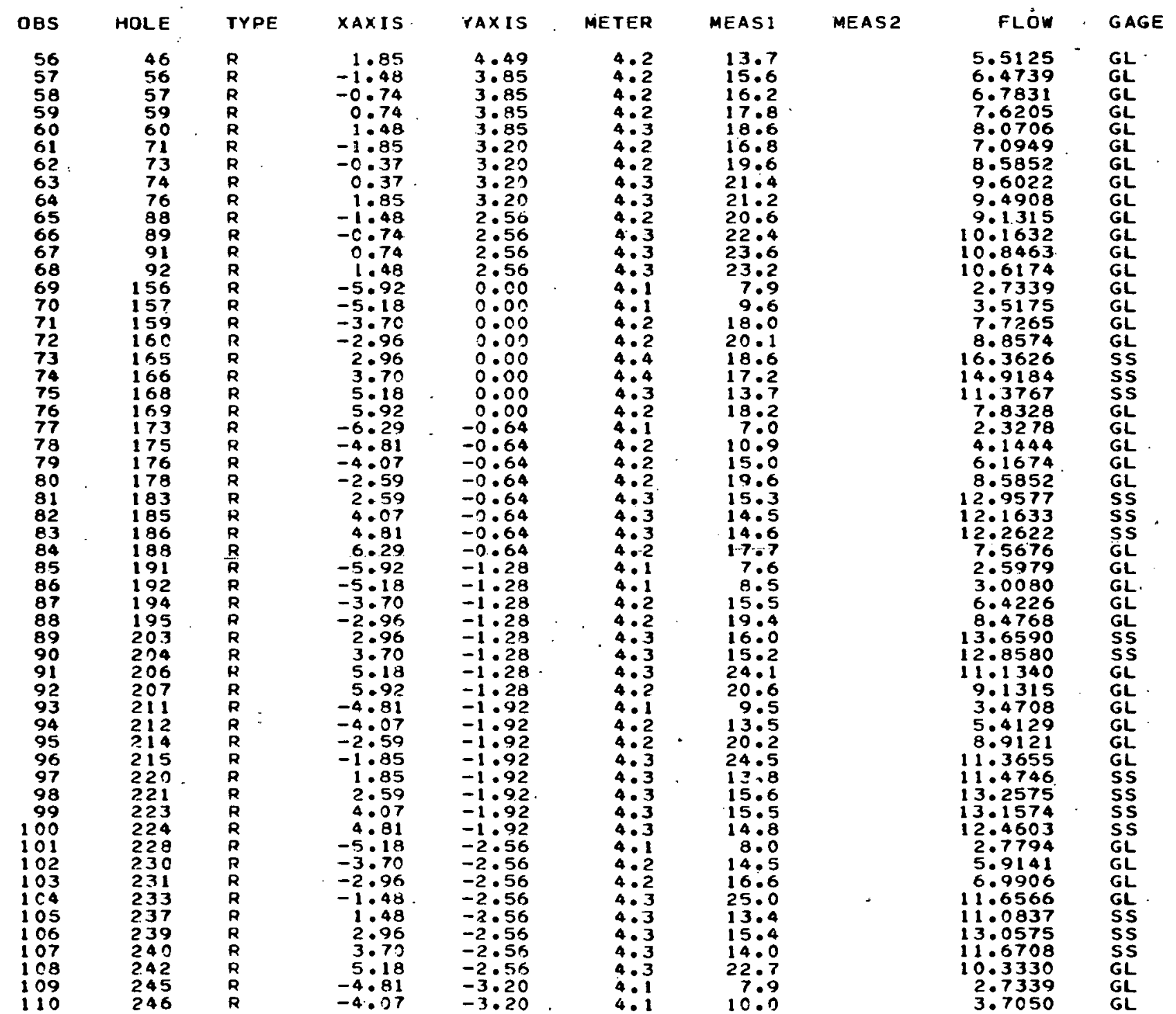


Table B-2. (continued)

RLUCK 1-1046 (4 PSIG)

\begin{tabular}{|c|c|c|c|c|c|c|c|c|c|}
\hline OBS & HOLE & TYPE & XAXIS & YAXIS & NE.TER & MEASI & MEAS 2 & FLOW & GAGE \\
\hline $\begin{array}{l}1111 \\
111 \\
1113 \\
1114 \\
115 \\
1116 \\
1117 \\
1118 \\
119 \\
1120 \\
1121 \\
122 \\
1123 \\
124 \\
125 \\
126 \\
127 \\
128 \\
129 \\
131 \\
131 \\
132 \\
133 \\
134 \\
135 \\
136 \\
137 \\
138 \\
139 \\
140 \\
141 \\
142 \\
143 \\
144 \\
145 \\
146\end{array}$ & $\begin{array}{l}249 \\
249 \\
254 \\
255 \\
257 \\
258 \\
262 \\
263 \\
271 \\
272 \\
276 \\
278 \\
285 \\
287 \\
290 \\
291 \\
299 \\
300 \\
107 \\
108 \\
124 \\
128 \\
143 \\
146 \\
179 \\
182 \\
197 \\
201 \\
217 \\
218 \\
125 \\
127 \\
162 \\
163 \\
198 \\
200\end{array}$ & 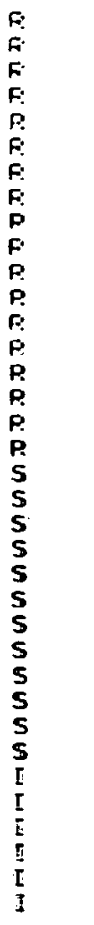 & $\begin{array}{r}-2.59 \\
-1.85 \\
1.85 \\
2.59 \\
4.77 \\
4.81 \\
-3.70 \\
-2.96 \\
2.96 \\
3.70 \\
-4.07 \\
-2.59 \\
2.59 \\
4.07 \\
-3.70 \\
-2.96 \\
2.96 \\
3: 70 \\
-0.37 \\
0.37 \\
-1.48 \\
1.48 \\
-1.85 \\
1.85 \\
-1.85 \\
1.855 \\
-1.48 \\
1.48 \\
-0.37 \\
0.37 \\
-0.74 \\
0.74 \\
-1.48 \\
1.48 \\
-0.74 \\
0.74\end{array}$ & $\begin{array}{r}-3.20 \\
-3.20 \\
-3.20 \\
-3.20 \\
-3.20 \\
-3.20 \\
-3.85 \\
-3.85 \\
-3.85 \\
-3.85 \\
-3.85 \\
-4.49 \\
-4.49 \\
-4.49 \\
-4.86 \\
-5.13 \\
-5.13 \\
-5.13 \\
-5.13 \\
1.92 \\
1.92 \\
1.28 \\
1.28 \\
0.64 \\
0.64 \\
-0.64 \\
-0.64 \\
-1.28 \\
-1.28 \\
-1.292 \\
-1.92 \\
1.28 \\
1.28 \\
0.00 \\
0.00 \\
-1.28 \\
-1.28\end{array}$ & $\begin{array}{l}4 \cdot 2 \\
4: 2 \\
4: 3 \\
4: 3 \\
4: 3 \\
4: 3 \\
4: 1 \\
4: 2 \\
4: 3 \\
4: 3 \\
4: 1 \\
4: 2 \\
4: 3 \\
4: 3 \\
4: 1 \\
4: 1 \\
4: 2 \\
4: 2 \\
4: 2 \\
4: 3 \\
4: 3 \\
4: 4 \\
4: 3 \\
4: 4 \\
4: 3 \\
4: 3 \\
4: 3 \\
4: 3 \\
4: 3 \\
4: 3 \\
4: 3 \\
4: 3 \\
4: 3 \\
4: 3 \\
4: 3 \\
4 \cdot 3\end{array}$ & $\begin{array}{l}17.0 \\
20: 5 \\
15.5 \\
15.0 \\
13.2 \\
21.3 \\
10.2 \\
12.9 \\
14.0 \\
13.0 \\
7.6 \\
12.4 \\
12.5 \\
17.3 \\
6.6 \\
8.1 \\
10.1 \\
16.3 \\
23.1 \\
14.2 \\
14.1 \\
16.5 \\
13.8 \\
16.5 \\
13.8 \\
14.0 \\
14.7 \\
13.1 \\
15.3 \\
16.0 \\
21.8 \\
24.5 \\
99.7 \\
11.4 \\
10.0 \\
10.2\end{array}$ & ' & $\begin{array}{r}7.1994 \\
9.0765 \\
13.1574 \\
12.6589 \\
10.8890 \\
9.5465 \\
3.7991 \\
5.1157 \\
11.6708 \\
10.6947 \\
2.5979 \\
4.8701 \\
10.2111 \\
7.3567 \\
2.1492 \\
2.8250 \\
7.9074 \\
6.8348 \\
10.5297 \\
11.8675 \\
11.7691 \\
14.2051 \\
11.4746 \\
14.2051 \\
11.4746 \\
11.6708 \\
12.3612 \\
10.7918 \\
12.9577 \\
13.6590 \\
9.7969 \\
11.3655 \\
11.3611 \\
13.7028 \\
11.7707 \\
12.0446\end{array}$ & $\begin{array}{l}G L \\
G L \\
S S \\
S S \\
S S \\
G L \\
G L \\
G L \\
S S \\
S S \\
G L \\
G L \\
S S \\
G L \\
G L \\
G L \\
S S \\
G L \\
G L \\
S S \\
S S \\
S S \\
S S \\
S S \\
S S \\
S S \\
S S \\
S S \\
S S \\
S S \\
G L \\
G L \\
T A \\
T A \\
\text { TA } \\
\text { TA }\end{array}$ \\
\hline
\end{tabular}


Table B-2. (continued)

BLOCK 1-1343 (4 PSIG)

\begin{tabular}{|c|c|c|c|c|c|c|c|c|c|}
\hline OBS & HCLE & TYPE & XAXIS & YAXIS & ME TER & MEASI & MEASZ & FLOW & GAGE \\
\hline $\begin{array}{l}1 \\
2 \\
3 \\
4 \\
5 \\
6 \\
7 \\
8 \\
9 \\
10 \\
11 \\
12 \\
13 \\
14 \\
15 \\
16 \\
17 \\
19 \\
19 \\
20 \\
21 \\
22 \\
23 \\
24 \\
25 \\
26 \\
27 \\
28 \\
29 \\
30 \\
31 \\
32 \\
33 \\
34 \\
35 \\
36 \\
37 \\
38 \\
39 \\
40 \\
41 \\
42 \\
43 \\
44 \\
45 \\
46 \\
47 \\
48 \\
49 \\
50 \\
51 \\
52 \\
53 \\
54 \\
55\end{array}$ & 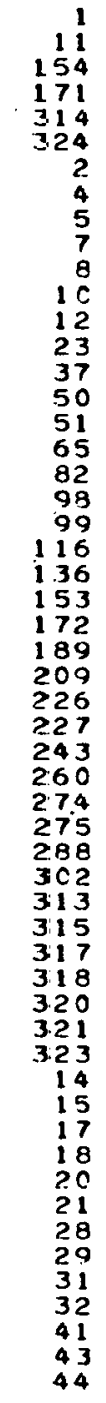 & $\begin{array}{l}C \\
C \\
C \\
C \\
C \\
C \\
F \\
F \\
F \\
F \\
F \\
F \\
F \\
F \\
F \\
F \\
F \\
F \\
F \\
F \\
F \\
F \\
F \\
F \\
F \\
F \\
F \\
F \\
F \\
F \\
F \\
F \\
F \\
F \\
F \\
F \\
F \\
F \\
F \\
F \\
F \\
F \\
R \\
R \\
R \\
R \\
R \\
R \\
R \\
R \\
R \\
R \\
R \\
R \\
R\end{array}$ & $\begin{array}{r}-3.79 \\
3.70 \\
-7.40 \\
7.40 \\
-3: 70 \\
3.70 \\
-2.96 \\
-1.49 \\
-0: 74 \\
0: 74 \\
1.43 \\
2.96 \\
-4.07 \\
4.07 \\
-4.81 \\
4.81 \\
-5.18 \\
5.13 \\
-5.92 \\
5.92 \\
-6.29 \\
6.29 \\
-7.03 \\
7.03 \\
-7.03 \\
7.03 \\
-6.29 \\
6.29 \\
-5.92 \\
5.92 \\
-5.18 \\
5.18 \\
-4.81 \\
4.811 \\
-4.07 \\
4.07 \\
-2.96 \\
-1.48 \\
-0.74 \\
0.74 \\
1.48 \\
2.96 \\
-2.59 \\
-1.85 \\
-0.37 \\
0.37 \\
1.85 \\
2.59 \\
-1.48 \\
-0.74 \\
0.74 \\
1.49 \\
-1.85 \\
-0.37 \\
0.37\end{array}$ & $\begin{array}{r}6.41 \\
6.41 \\
0.00 \\
0.00 \\
-6.41 \\
-6.41 \\
6.41 \\
6.41 \\
6.41 \\
6.41 \\
6.41 \\
6.41 \\
5.77 \\
5.77 \\
4.49 \\
4.49 \\
3.85 \\
3.85 \\
2.56 \\
2.56 \\
1.92 \\
1.92 \\
0.84 \\
0.64 \\
-0.64 \\
-0.64 \\
-1.92 \\
-1.92 \\
-2.56 \\
-2.56 \\
-3.85 \\
-3.85 \\
-4.49 \\
-4.49 \\
-5.77 \\
-5.77 \\
-6.41 \\
-6.41 \\
-6.41 \\
-6.41 \\
-6.41 \\
-6.41 \\
5.77 \\
5.77 \\
5.77 \\
5.77 \\
5.77 \\
5.77 \\
5.13 \\
5.13 \\
5.13 \\
5.13 \\
4.49 \\
4.49 \\
4.49\end{array}$ & $\begin{array}{l}4: 2 \\
4: 2 \\
4: 2 \\
4: 2 \\
4: 2 \\
4: 2 \\
4: 2 \\
4: 2 \\
4: 2 \\
4: 2 \\
4: 2 \\
4: 2 \\
4: 2 \\
4: 2 \\
4: 2 \\
4: 2 \\
4: 2 \\
4: 2 \\
4: 2 \\
4: 2 \\
4: 2 \\
4: 2 \\
4: 2 \\
4: 2 \\
4: 2 \\
4: 2 \\
4: 22 \\
4: 2 \\
4: 2 \\
4: 2 \\
4: 2 \\
4: 2 \\
4: 2 \\
4: 22 \\
4: 2 \\
4: 2 \\
4: 2 \\
4: 2 \\
4: 2 \\
4: 2 \\
4: 2 \\
4: 2 \\
4: 2 \\
4: 3 \\
4: 3 \\
4: 3 \\
4: 2 \\
4: 3 \\
4: 33 \\
4: 3 \\
4: 3 \\
4: 3 \\
4: 3 \\
4: 3 \\
4: 3\end{array}$ & $\begin{array}{l}18.0 \\
15.5 \\
13.7 \\
16.4 \\
13.8 \\
14.5 \\
12.0 \\
12.7 \\
12.4 \\
13.5 \\
13.2 \\
11.4 \\
11.3 \\
10.8 \\
113.9 \\
12.5 \\
13.6 \\
11.6 \\
14.6 \\
10.7 \\
13.6 \\
11.4 \\
18.7 \\
10.6 \\
15.3 \\
10.3 \\
10.6 \\
10.6 \\
11.0 \\
16.4 \\
11.2 \\
17.9 \\
16.6 \\
18.1 \\
15.0 \\
16.4 \\
15.6 \\
18.4 \\
18.3 \\
19.3 \\
17.9 \\
17.3 \\
14.2 \\
15.0 \\
1.4 .6 \\
16.2 \\
14.2 \\
15.5 \\
16.0 \\
15.6 \\
15.6 \\
15.1 \\
15.5 \\
15.0 \\
15.5\end{array}$ & & $\begin{array}{r}7.7265 \\
6.4226 \\
5.5125 \\
6.8867 \\
5.5625 \\
5.9141 \\
9.77011 \\
10.3728 \\
10.0842 \\
11.1477 \\
10.3562 \\
9.1300 \\
9.0352 \\
8.5632 \\
11.5380 \\
10.1803 \\
11.2451 \\
9.3199 \\
12.2257 \\
8.4692 \\
11.2451 \\
9.1300 \\
8.0998 \\
8.3753 \\
6.3203 \\
8.0942 \\
8.3353 \\
8.3753 \\
8.7517 \\
6.8867 \\
8.9406 \\
7.6734 \\
6.9906 \\
7.7796 \\
6.1674 \\
6.8867 \\
6.1674 \\
7.9394 \\
7.8860 \\
8.4227 \\
7.6734 \\
7.3567 \\
11.8320 \\
12.6589 \\
12.2622 \\
13.8605 \\
11.8320 \\
13.1574 \\
13.6590 \\
13.2575 \\
13.2575 \\
2.7584 \\
13.1574 \\
12.6589 \\
13.1574\end{array}$ & $\begin{array}{l}G L \\
G L \\
G L \\
G L \\
G L \\
G L \\
S S \\
S S \\
S S \\
S S \\
S S \\
S S \\
S S \\
\text { SS } \\
\text { SS } \\
S S \\
S S \\
\text { SS } \\
S S \\
S S \\
S S \\
S S \\
G L \\
S S \\
G L \\
S S \\
S S \\
S S \\
S S \\
G L \\
S S \\
G L \\
G L \\
G L \\
G L \\
G L \\
G L \\
G L \\
G L \\
G L \\
G L \\
G L \\
\text { SS } \\
S S \\
S S \\
S S \\
\text { SS } \\
S S \\
S S \\
S S \\
S S \\
S S \\
S S \\
S S \\
S S\end{array}$ \\
\hline
\end{tabular}


Table B-2. (continued)

ELOCK $1-1343$ (4 PSIG)

\begin{tabular}{|c|c|c|c|c|c|c|c|c|c|}
\hline DBS & HOLE & TYPE & $X A \times 1 S$ & YAXIS & METER & MEASI & MEEAS 2 & FLOW & GAGE \\
\hline $\begin{array}{l}55 \\
57 \\
59 \\
59 \\
60 \\
61 \\
62 \\
63 \\
64 \\
65 \\
66 \\
67 \\
68 \\
69 \\
70 \\
71 \\
72 \\
73 \\
74 \\
75 \\
75 \\
77 \\
78 \\
79 \\
80 \\
81 \\
82 \\
83 \\
84 \\
85 \\
86 \\
87 \\
88 \\
89 \\
90 \\
91 \\
92 \\
93 \\
94 \\
95 \\
96 \\
97 \\
99 \\
99 \\
160 \\
101 \\
102 \\
103 \\
104 \\
105 \\
106 \\
107 \\
108 \\
109 \\
110\end{array}$ & $\begin{array}{l}46 \\
56 \\
57 \\
59 \\
65 \\
71 \\
73 \\
74 \\
75 \\
83 \\
59 \\
91 \\
92 \\
156 \\
157 \\
159 \\
160 \\
165 \\
156 \\
168 \\
159 \\
173 \\
175 \\
175 \\
173 \\
183 \\
185 \\
186 \\
188 \\
191 \\
192 \\
194 \\
195 \\
203 \\
204 \\
206 \\
207 \\
211 \\
212 \\
214 \\
215 \\
220 \\
221 \\
223 \\
224 \\
228 \\
230 \\
231 \\
233 \\
237 \\
239 \\
240 \\
242 \\
245 \\
246\end{array}$ & $\begin{array}{l}R \\
R \\
R \\
R \\
R \\
R \\
R \\
R \\
Q \\
R \\
R \\
R \\
R \\
R \\
R \\
R \\
R \\
R \\
R \\
R \\
R \\
R \\
R \\
R \\
R \\
R \\
R \\
R \\
R \\
R \\
R \\
R \\
R \\
R \\
R \\
R \\
R \\
R \\
R \\
R \\
R \\
R \\
R \\
R \\
R \\
R \\
R \\
R \\
R \\
R \\
R \\
R \\
P \\
R \\
R \\
R \\
R \\
R \\
R\end{array}$ & $\begin{array}{r}1.85 \\
-1.48 \\
-0.74 \\
0.74 \\
1.49 \\
-1.85 \\
-0.37 \\
0.37 \\
1.85 \\
-1.43 \\
-0.74 \\
0.74 \\
1.49 \\
-5.92 \\
-5.18 \\
-3.70 \\
-2.96 \\
2.96 \\
3.70 \\
5.13 \\
5.92 \\
-6.29 \\
-4.81 \\
-4.07 \\
-2.59 \\
2.59 \\
4.07 \\
4.81 \\
6.29 \\
-5.92 \\
-5.18 \\
-3.70 \\
-2.96 \\
2.96 \\
3.70 \\
5.18 \\
5.92 \\
-4.81 \\
-4.07 \\
-2.59 \\
-1.85 \\
1.85 \\
2.59 \\
4.07 \\
4.81 \\
-5.18 \\
-3.70 \\
-2.96 \\
-1.48 \\
1.48 \\
2.96 \\
3.70 \\
5.18 \\
-4.81 \\
-4.07\end{array}$ & 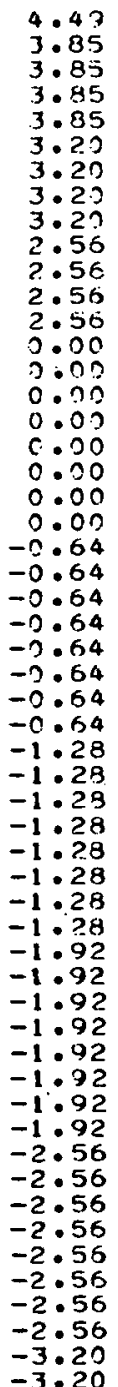 & $\begin{array}{l}4 \cdot 3 \\
4: 3 \\
4: 3 \\
4: 3 \\
4: 3 \\
4: 3 \\
4: 3 \\
4: 3 \\
4: 33 \\
4: 33 \\
4: 3 \\
4: 3 \\
4: 3 \\
4: 3 \\
4: 4 \\
4: 4 \\
4: 3 \\
4: 4 \\
4: 4 \\
4: 3 \\
4: 3 \\
4: 3 \\
4: 3 \\
4: 4 \\
4: 3 \\
4: 4 \\
4: 3 \\
4: 3 \\
4: 32 \\
4: 3 \\
4: 3 \\
4: 44 \\
4: 3 \\
4: 3 \\
4: 3 \\
4: 22 \\
4: 3 \\
4: 3 \\
4: 3 \\
4: 3 \\
4: 3 \\
4: 3 \\
4: 4 \\
4: 3 \\
4: 3 \\
4: 2 \\
4: 3 \\
4: 3 \\
4: 3 \\
4: 3 \\
4: 3 \\
4: 3 \\
4: 2 \\
4: 2 \\
4: 3\end{array}$ & $\begin{array}{l}15.3 \\
14.7 \\
14.7 \\
15.2 \\
15.0 \\
15.2 \\
15.4 \\
15.9 \\
15.8 \\
15.1 \\
14.9 \\
15.6 \\
15.6 \\
15.4 \\
17.0 \\
17.7 \\
15.8 \\
15.9 \\
18.2 \\
15.0 \\
14.1 \\
13.8 \\
16.4 \\
17.2 \\
16.1 \\
17.0 \\
15.8 \\
15.5 \\
12.5 \\
13.6 \\
15.2 \\
17.8 \\
16.8 \\
16.5 \\
15.2 \\
13.9 \\
11.1 \\
15.2 \\
16.1 \\
15.8 \\
16.6 \\
15.6 \\
17.2 \\
15.5 \\
14.1 \\
13.1 \\
15.8 \\
15.6 \\
16.0 \\
15.7 \\
16.7 \\
15.0 \\
11.5 \\
13.4 \\
15.0\end{array}$ & & $\begin{array}{l}12.9577 \\
12.3612 \\
12.3612 \\
12.8530 \\
12.6589 \\
12.8580 \\
13.0575 \\
13.5584 \\
13.4590 \\
12.7584 \\
12.5595 \\
13.2575 \\
13.2575 \\
13.0575 \\
14.7140 \\
15.4315 \\
13.4580 \\
14.6120 \\
15.9476 \\
12.6589 \\
11.7691 \\
11.4403 \\
14.0624 \\
14.9184 \\
13.7597 \\
14.7140 \\
13.4580 \\
13.1574 \\
10.1803 \\
11.24451 \\
12.8580 \\
15.5345 \\
14.4677 \\
14.1635 \\
12.8580 \\
11.5380 \\
8.8461 \\
12.8580 \\
13.7597 \\
13.4580 \\
14.2648 \\
13.2575 \\
14.9184 \\
13.1574 \\
11.7691 \\
110.7593 \\
13.4580 \\
13.2575 \\
13.6590 \\
13.3577 \\
12.3662 \\
9.6589 \\
11.02494 \\
112.6589\end{array}$ & 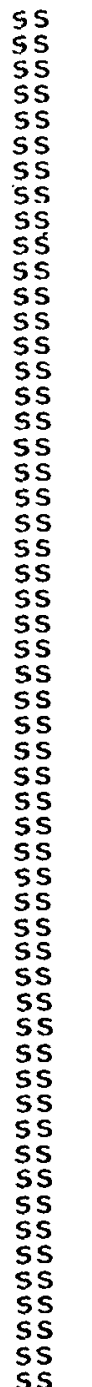 \\
\hline
\end{tabular}


Table B-2. (continued)

BLOCK I-1343 (4 PSIG)

\begin{tabular}{|c|c|c|c|c|c|c|c|c|c|}
\hline OBS & HOLE & TYPE & XAXIS & YAXIS & METER & MEASI & MEAS 2 & FLOW & GAGE \\
\hline 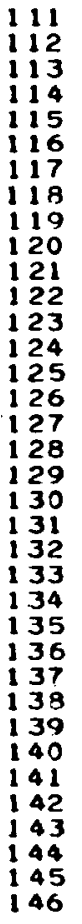 & $\begin{array}{l}248 \\
249 \\
254 \\
255 \\
257 \\
258 \\
262 \\
263 \\
271 \\
272 \\
276 \\
278 \\
285 \\
287 \\
296 \\
291 \\
299 \\
300 \\
107 \\
108 \\
124 \\
128 \\
143 \\
146 \\
179 \\
182 \\
199 \\
201 \\
217 \\
218 \\
125 \\
127 \\
162 \\
163 \\
198 \\
200\end{array}$ & $\begin{array}{l}R \\
R \\
R \\
R \\
R \\
R \\
R \\
R \\
R \\
R \\
R \\
R \\
R \\
R \\
R \\
R \\
R \\
R \\
R \\
S \\
S \\
S \\
S \\
S \\
S \\
S \\
S \\
S \\
S \\
S \\
S \\
I \\
I \\
I \\
I \\
I \\
I\end{array}$ & $\begin{array}{r}-2.59 \\
-1.85 \\
1.85 \\
2.59 \\
4.07 \\
4.81 \\
-3.70 \\
-2.96 \\
2.96 \\
3.70 \\
-4.07 \\
-2.59 \\
2.59 \\
4.07 \\
-3.70 \\
-2.96 \\
2.96 \\
3.70 \\
-0.37 \\
0.37 \\
-1.48 \\
1.48 \\
-1.85 \\
1.85 \\
-1.85 \\
1.85 \\
-1.48 \\
1.48 \\
-0.37 \\
0.37 \\
-0.74 \\
0.74 \\
-1.43 \\
1.48 \\
-0.74 \\
0.74\end{array}$ & $\begin{array}{r}-3.20 \\
-3.20 \\
-3.20 \\
-3.20 \\
-3.20 \\
-3.20 \\
-3.85 \\
-3.85 \\
-3.85 \\
-3.85 \\
-4.49 \\
-4.49 \\
-4.49 \\
-4.86 \\
-5.13 \\
-5.13 \\
-5.13 \\
-5.13 \\
1.92 \\
1.92 \\
1.28 \\
1.28 \\
0.64 \\
0.64 \\
-0.64 \\
-0.64 \\
-1.28 \\
-1.28 \\
-1.92 \\
-1.92 \\
1.28 \\
1.29 \\
0.00 \\
0.00 \\
-1.28 \\
-1.28\end{array}$ & 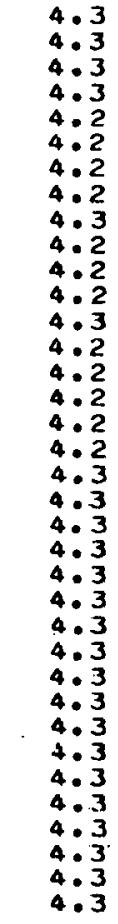 & $\begin{array}{l}14.5 \\
16.2 \\
16.5 \\
16.8 \\
13.4 \\
11.5 \\
14.1 \\
14.4 \\
14.9 \\
13.5 \\
12.4 \\
13.1 \\
14.2 \\
11.7 \\
11.8 \\
12.1 \\
13.1 \\
10.6 \\
15.1 \\
15.7 \\
15.5 \\
15.4 \\
16.0 \\
15.6 \\
16.4 \\
16.7 \\
16.7 \\
16.8 \\
15.8 \\
17.1 \\
13.8 \\
14.9 \\
14.6 \\
15.8 \\
16.2 \\
16.3\end{array}$ & & $\begin{array}{l}12.1633 \\
13.8605 \\
14.1635 \\
14.4677 \\
11.0504 \\
9.2249 \\
11.7339 \\
12.0286 \\
12.5595 \\
11.1477 \\
10.0842 \\
10.7593 \\
11.8675 \\
9.4150 \\
9.5102 \\
9.7967 \\
10.7593 \\
8.3753 \\
12.7584 \\
13.3577 \\
13.1574 \\
13.0575 \\
13.6590 \\
13.2575 \\
14.0624 \\
14.3662 \\
14.3662 \\
14.4677 \\
13.4580 \\
14.7730 \\
11.4746 \\
12.5595 \\
12.2622 \\
13.4580 \\
13.8605 \\
13.9614\end{array}$ & $\begin{array}{l}\text { SS } \\
\text { SS } \\
\text { SS } \\
\text { SS } \\
\text { SS } \\
\text { SS } \\
\text { SS } \\
\text { SS } \\
\text { SS } \\
\text { SS } \\
\text { SS } \\
\text { SS } \\
\text { SS } \\
\text { SS } \\
\text { sS } \\
\text { SS } \\
\text { SS } \\
\text { SS } \\
\text { SS } \\
\text { SS } \\
\text { SS } \\
\text { SS } \\
\text { SS } \\
\text { SS } \\
\text { SS } \\
\text { SS } \\
\text { SS } \\
\text { SS } \\
\text { SS } \\
\text { SS } \\
\text { SS } \\
\text { SS } \\
\text { SS } \\
\text { SS } \\
\text { SS } \\
\text { SS }\end{array}$ \\
\hline
\end{tabular}


Table B-2. (continued)

PLOCK 1-16̣?? (4 PSIG)

\begin{tabular}{|c|c|c|c|c|c|c|c|c|c|}
\hline OBS & HOLE & TYODE & $X A \times I S$ & YAXIS & METER & MEASI & MEAS2 & FLOW & GAGE \\
\hline $\begin{array}{l}1 \\
2 \\
3 \\
4 \\
5 \\
6 \\
7 \\
8 \\
9 \\
10 \\
11 \\
12 \\
13 \\
14 \\
15 \\
16 \\
17 \\
19 \\
19 \\
20 \\
21 \\
22 \\
23 \\
24 \\
25 \\
26 \\
27 \\
28 \\
29 \\
30 \\
31 \\
32 \\
33 \\
34 \\
35 \\
36 \\
37 \\
38 \\
39 \\
40 \\
41 \\
42 \\
43 \\
44 \\
45 \\
46 \\
47 \\
48 \\
49 \\
50 \\
51 \\
52 \\
53 \\
54 \\
55\end{array}$ & $\begin{array}{r}11 \\
11 \\
154 \\
171 \\
314 \\
324 \\
2 \\
4 \\
5 \\
7 \\
7 \\
8 \\
11 \\
12 \\
23 \\
37 \\
50 \\
51 \\
65 \\
82 \\
98 \\
99 \\
116 \\
136 \\
153 \\
172 \\
189 \\
269 \\
226 \\
227 \\
243 \\
260 \\
274 \\
275 \\
288 \\
302 \\
313 \\
315 \\
317 \\
318 \\
320 \\
321 \\
323 \\
14 \\
15 \\
117 \\
118 \\
20 \\
21 \\
218 \\
29 \\
31 \\
32 \\
41 \\
43 \\
44 \\
\end{array}$ & $\begin{array}{l}C \\
C \\
C \\
C \\
C \\
C \\
F \\
F \\
F \\
F \\
F \\
F \\
F \\
F \\
F \\
F \\
F \\
F \\
F \\
F \\
F \\
F \\
F \\
F \\
F \\
F \\
F \\
F \\
F \\
F \\
F \\
F \\
F \\
F \\
F \\
F \\
F \\
F \\
F \\
F \\
F \\
F \\
R \\
R \\
R \\
R \\
R \\
R \\
R \\
R \\
R \\
R \\
R \\
R \\
R\end{array}$ & $\begin{array}{r}-3.70 \\
3.70 \\
-7.40 \\
7.40 \\
-3.79 \\
3.70 \\
-2.95 \\
-1.48 \\
-0.74 \\
0.74 \\
1.49 \\
2.99 \\
-4.07 \\
4.07 \\
-4.81 \\
4.81 \\
-5.18 \\
5.18 \\
-5.92 \\
5.99 \\
-6.29 \\
6.29 \\
-7.03 \\
7.03 \\
-7.03 \\
7.03 \\
-6.29 \\
6.29 \\
-5.92 \\
5.92 \\
-5.18 \\
5.18 \\
-4.81 \\
4.81 \\
-4.07 \\
4.07 \\
-2.95 \\
-1.49 \\
-0.74 \\
0.74 \\
1.48 \\
2.96 \\
-2.59 \\
-1.85 \\
-0.37 \\
0.37 \\
1.85 \\
2.59 \\
-1.49 \\
-0.74 \\
0.74 \\
1.48 \\
-1.85 \\
-0.37 \\
0.37\end{array}$ & $\begin{array}{r}5.409 \\
6.409 \\
6.000 \\
0.000 \\
-6.409 \\
-6.409 \\
6.409 \\
6.409 \\
6.409 \\
6.409 \\
5.409 \\
6.409 \\
5.768 \\
5.768 \\
4.486 \\
4.486 \\
3.845 \\
3.845 \\
2.563 \\
2.553 \\
1.923 \\
1.923 \\
0.641 \\
2.641 \\
-0.641 \\
-0.641 \\
-1.923 \\
-1.923 \\
-2.563 \\
-2.563 \\
-3.845 \\
-3.845 \\
-4.486 \\
-4.486 \\
-5.768 \\
-5.768 \\
-5.409 \\
-6.409 \\
-6.409 \\
-6.409 \\
-6.409 \\
-6.409 \\
5.768 \\
5.769 \\
5.768 \\
5.768 \\
5.768 \\
5.768 \\
5.127 \\
5.127 \\
5.127 \\
5.127 \\
4.486 \\
4.496 \\
4.486\end{array}$ & 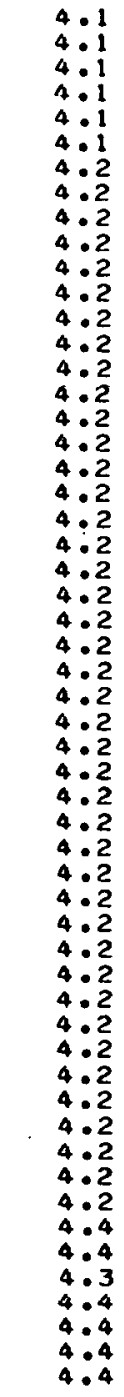 & $\begin{array}{l}7.7 \\
10.4 \\
10.0 \\
71.0 \\
12.0 \\
9.6 \\
111.6 \\
17.0 \\
17.9 \\
17.3 \\
15.7 \\
12.3 \\
10.6 \\
13.9 \\
12.8 \\
16.9 \\
13.0 \\
17.2 \\
14.0 \\
14.0 \\
13.3 \\
12.1 \\
11.4 \\
18: 7 \\
12.9 \\
8.1 \\
16.1 \\
18.9 \\
18.1 \\
9.0 \\
18.8 \\
10.5 \\
17.6 \\
10.7 \\
16.0 \\
11.0 \\
14.3 \\
16.3 \\
17.4 \\
18.4 \\
16.2 \\
12.9 \\
12.8 \\
14.6 \\
15.1 \\
15.2 \\
14.2 \\
12.2 \\
16.4 \\
17.2 \\
16.9 \\
17.0 \\
17.5 \\
18.3 \\
19.2\end{array}$ & . & $\begin{array}{l}2.6432 \\
3.8936 \\
3.7050 \\
2.3278 \\
4.6601 \\
3.5175 \\
4.4810 \\
7.1994 \\
7.6734 \\
7.3567 \\
6.5252 \\
4.8212 \\
4.0013 \\
5.6125 \\
5.0665 \\
7.1471 \\
5.1651 \\
7.3042 \\
5.6625 \\
5.6625 \\
5.3135 \\
4.7237 \\
4.3845 \\
3.1102 \\
5.1157 \\
2.8344 \\
6.7313 \\
3.70728 \\
7.7796 \\
3.2491 \\
8.1535 \\
3.9537 \\
7.5147 \\
4.0489 \\
6.6797 \\
4.1923 \\
5.8132 \\
6.8348 \\
7.4093 \\
7.9394 \\
6.7831 \\
5.1157 \\
10.4693 \\
12.2257 \\
12.7205 \\
12.8199 \\
11.8320 \\
9.8924 \\
14.1037 \\
14.9184 \\
14.5693 \\
14.7140 \\
15.2259 \\
16.0512 \\
16.9888\end{array}$ & $\begin{array}{l}G L \\
G L \\
G L \\
G L \\
G L \\
G L \\
G L \\
G L \\
G L \\
G L \\
G L \\
G L \\
G L \\
G L \\
G L \\
G L \\
G L \\
G L \\
G L \\
G L \\
G L \\
G L \\
G L \\
G L \\
G L \\
G L \\
G L \\
G L \\
G L \\
G L \\
G L \\
G L \\
G L \\
G L \\
G L \\
G L \\
G L \\
G L \\
G L \\
G L \\
G L \\
G L \\
S S \\
S S \\
S S \\
S S \\
S S \\
S S \\
S S \\
S S \\
S S \\
S S \\
S S \\
S S \\
S S\end{array}$ \\
\hline
\end{tabular}


Table B-2. (continued)

BLOCK 1-1622 (4 PSIG)

\begin{tabular}{|c|c|c|c|c|c|c|c|c|c|}
\hline oBS & HOLE & TYPE & $X A X I S$ & YAXIS & METER & MEASI & MEAS2 & FLOW & GAGE \\
\hline $\begin{array}{l}56 \\
57 \\
58 \\
59 \\
60 \\
61 \\
62 \\
63 \\
64 \\
65 \\
66 \\
67 \\
68 \\
69 \\
70 \\
71 \\
72 \\
73 \\
74 \\
75 \\
76 \\
77 \\
78 \\
79 \\
80 \\
81 \\
82 \\
83 \\
84 \\
85 \\
86 \\
87 \\
88 \\
89 \\
90 \\
91 \\
92 \\
93 \\
94 \\
95 \\
96 \\
97 \\
98 \\
99 \\
100 \\
101 \\
102 \\
103 \\
104 \\
105 \\
106 \\
107 \\
108 \\
109 \\
110\end{array}$ & $\begin{array}{l}46 \\
56 \\
57 \\
59 \\
60 \\
71 \\
73 \\
74 \\
76 \\
88 \\
89 \\
91 \\
92 \\
156 \\
157 \\
159 \\
160 \\
165 \\
166 \\
168 \\
169 \\
173 \\
175 \\
176 \\
178 \\
183 \\
185 \\
186 \\
198 \\
191 \\
192 \\
194 \\
195 \\
203 \\
204 \\
206 \\
207 \\
211 \\
212 \\
214 \\
215 \\
220 \\
221 \\
223 \\
224 \\
228 \\
230 \\
231 \\
233 \\
237 \\
239 \\
240 \\
242 \\
245 \\
246\end{array}$ & $\begin{array}{l}R \\
R \\
R \\
R \\
R \\
R \\
R \\
R \\
R \\
R \\
R \\
R \\
R \\
P \\
R \\
R \\
R \\
R \\
R \\
R \\
R \\
R \\
R \\
R \\
R \\
R \\
R \\
R \\
R \\
R \\
R \\
R \\
R \\
R \\
P \\
R \\
R \\
R \\
R \\
R \\
R \\
R \\
R \\
R \\
R \\
R \\
R \\
R \\
R \\
R \\
P \\
R \\
R \\
R \\
R \\
R \\
R \\
R \\
R \\
R\end{array}$ & $\begin{array}{r}1.85 \\
-1.48 \\
-0.74 \\
0.74 \\
1.48 \\
-1.85 \\
-0.37 \\
0.37 \\
1.85 \\
-1: 49 \\
-0.74 \\
0.74 \\
1.48 \\
-5.92 \\
-5.18 \\
-3.70 \\
-2.95 \\
2.96 \\
3.70 \\
5.18 \\
5.92 \\
-6.29 \\
-4.81 \\
-4.07 \\
-2.59 \\
2.59 \\
4.07 \\
4.81 \\
6.29 \\
-5.92 \\
-5.18 \\
-3.70 \\
-2.96 \\
2.96 \\
3.70 \\
5.18 \\
5.92 \\
-4.81 \\
-4.077 \\
-2.59 \\
-1.85 \\
1.85 \\
2.59 \\
4.07 \\
4.81 \\
-5.18 \\
-3.70 \\
-2.96 \\
-1.48 \\
1.49 \\
2.96 \\
3.70 \\
5.19 \\
-4.81 \\
-4.07\end{array}$ & 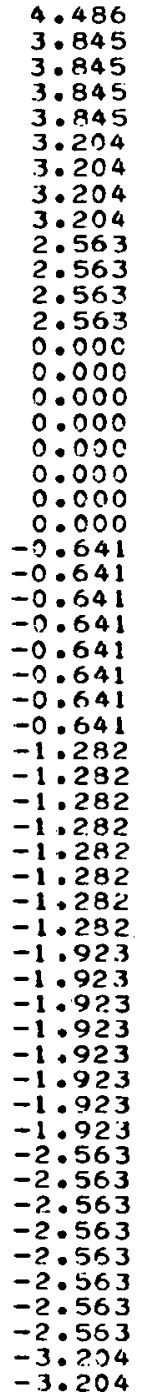 & $\begin{array}{l}4 \cdot 4 \\
4: 4 \\
4: 4 \\
4: 4 \\
4: 4 \\
4: 4 \\
4: 4 \\
4: 4 \\
4: 4 \\
4: 4 \\
4: 4 \\
4: 4 \\
4: 4 \\
4: 2 \\
4: 4 \\
4: 4 \\
4: 4 \\
4: 4 \\
4: 4 \\
4: 2 \\
4: 2 \\
4: 2 \\
4: 4 \\
4: 4 \\
4: 4 \\
4: 2 \\
4: 2 \\
4: 2 \\
4: 2 \\
4: 2 \\
4: 4 \\
4: 4 \\
4: 4 \\
4: 4 \\
4: 4 \\
4: 2 \\
4: 2 \\
4: 4 \\
4: 4 \\
4: 4 \\
4: 4 \\
4: 4 \\
4: 4 \\
4: 2 \\
4: 2 \\
4: 4 \\
4: 4 \\
4: 4 \\
4: 4 \\
4: 4 \\
4: 4 \\
4: 2 \\
4: 2 \\
4: 4 \\
4 \cdot 4 \\
\end{array}$ & 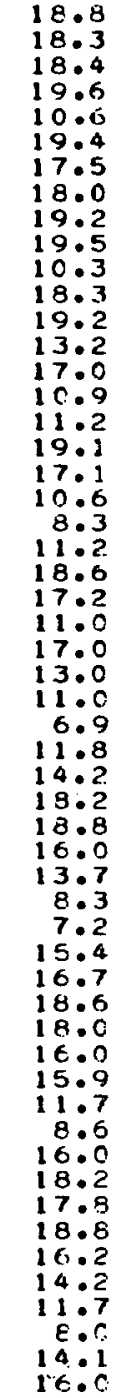 & $\begin{array}{l}12.5 \\
12.2 \\
12.8 \\
13.3 \\
13.0\end{array}$ & $\begin{array}{r}16.5709 \\
16.0512 \\
16.1549 \\
17.4086 \\
19.6688 \\
17.1985 \\
15.2259 \\
15.7408 \\
16.9888 \\
17.3035 \\
18.0966 \\
16.0512 \\
16.9888 \\
10.8562 \\
1.41140 \\
19.2431 \\
20.0146 \\
16.8841 \\
14.8162 \\
8.3753 \\
6.2482 \\
8.9406 \\
16.3626 \\
14.9184 \\
19.5322 \\
14.6282 \\
10.6625 \\
8.7517 \\
4.9845 \\
9.5102 \\
11.9029 \\
15.9476 \\
16.5709 \\
13.6992 \\
11.4108 \\
5.2482 \\
5.2533 \\
13.9961 \\
17.4083 \\
15.3626 \\
15.7408 \\
13.6992 \\
13.5984 \\
9.4150 \\
5.5220 \\
13.6992 \\
15.9476 \\
15.5345 \\
15.5709 \\
13.9012 \\
11.9029 \\
9.4150 \\
5.9754 \\
11.8042 \\
13.6992\end{array}$ & 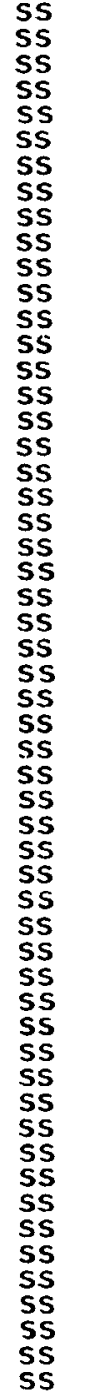 \\
\hline
\end{tabular}


Table B-2. (continued)

PLOCK 1-1622 (4 PSIG)

\begin{tabular}{|c|c|c|c|c|c|c|c|c|c|}
\hline DES & HDLE & TYPE & XAXIS & YAXIS & METER & MEAS 1 & MEASZ & FLOW & GAGE \\
\hline 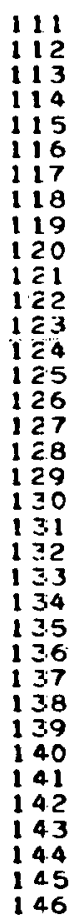 & $\begin{array}{l}248 \\
249 \\
254 \\
255 \\
257 \\
258 \\
262 \\
263 \\
271 \\
272 \\
276 \\
278 \\
285 \\
287 \\
290 \\
291 \\
299 \\
300 \\
107 \\
108 \\
124 \\
128 \\
143 \\
146 \\
179 \\
182 \\
197 \\
201 \\
217 \\
218 \\
125 \\
127 \\
162 \\
163 \\
198 \\
260\end{array}$ & $\begin{array}{l}P \\
R \\
R \\
R \\
R \\
R \\
R \\
R \\
R \\
R \\
R \\
R \\
R \\
R \\
R \\
R \\
R \\
R \\
R \\
S \\
S \\
S \\
S \\
S \\
S \\
S \\
S \\
S \\
S \\
S \\
S \\
I \\
I \\
I \\
I \\
I \\
I\end{array}$ & $\begin{array}{r}-2.59 \\
-1.85 \\
1.85 \\
2.59 \\
4.07 \\
4.81 \\
-3.70 \\
-2.96 \\
2.96 \\
3.70 \\
-4.07 \\
-2.59 \\
2.099 \\
4.07 \\
-3.70 \\
-2.96 \\
2.96 \\
3.70 \\
-0.37 \\
0.37 \\
-1.43 \\
1.48 \\
-1.85 \\
1.85 \\
-1.85 \\
1.85 \\
-1.48 \\
1.48 \\
-0.37 \\
0.37 \\
-0.74 \\
0.74 \\
-1.48 \\
1.48 \\
-0.74 \\
0.74\end{array}$ & $\begin{array}{r}-3.204 \\
-3.204 \\
-3.204 \\
-3.204 \\
-3.304 \\
-3.204 \\
-3.845 \\
-3.845 \\
-3.845 \\
-3.845 \\
-4.486 \\
-4.486 \\
-4.0486 \\
-4.486 \\
-5.127 \\
-5.127 \\
-5.127 \\
-5.127 \\
1.923 \\
1.923 \\
1.282 \\
1.282 \\
0.641 \\
0.641 \\
-0.641 \\
-0.641 \\
-1.282 \\
-1.282 \\
-1.923 \\
-1.923 \\
1.282 \\
10.282 \\
0.000 \\
0.000 \\
-1.232 \\
-1.282\end{array}$ & 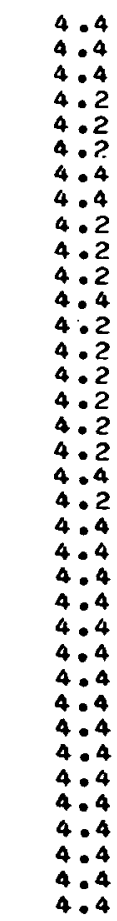 & $\begin{array}{l}17.1 \\
17.1 \\
15.5 \\
13.3 \\
9.8 \\
7.6 \\
15.2 \\
15.1 \\
12.5 \\
9.9 \\
12.1 \\
14.5 \\
11.8 \\
18.0 \\
12.2 \\
12.6 \\
10.5 \\
8.2 \\
10.7 \\
19.7 \\
11.4 \\
19.6 \\
11.5 \\
10.4 \\
20.0 \\
18.4 \\
10.5 \\
16.9 \\
19.5 \\
18.5 \\
19.1 \\
18.1 \\
11.0 \\
20.0 \\
18.6 \\
18.4\end{array}$ & $\begin{array}{l}19.3 \\
13.4 \\
13.6 \\
12.3 \\
12.4\end{array}$ & $\begin{array}{r}14.8162 \\
14.8162 \\
13.1963 \\
10.9533 \\
7.6282 \\
5.6134 \\
12.8961 \\
12.7962 \\
10.1803 \\
7.7212 \\
9.7967 \\
11.7957 \\
9.5102 \\
5.9754 \\
9.8924 \\
10.2765 \\
8.2814 \\
6.1571 \\
25.6148 \\
17.4136 \\
20.3028 \\
17.4086 \\
20.5940 \\
18.2871 \\
17.8304 \\
16.1549 \\
18.4778 \\
14.6120 \\
17.3035 \\
16.2587 \\
16.8841 \\
15.8441 \\
19.5322 \\
17.8304 \\
16.3626 \\
16.1549\end{array}$ & 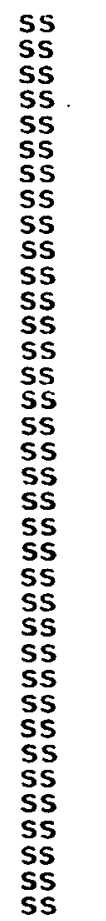 \\
\hline
\end{tabular}


Table B-2. (continued)

BLOCK 1-2926 (4 PSIG)

\begin{tabular}{|c|c|c|c|c|c|c|c|c|c|}
\hline OBS & HOLE & TYPE & XAXIS & YAXIS & METER & MEASI & MEAS 2 & FLOW & GAGE \\
\hline $\begin{array}{l}1 \\
2 \\
3 \\
4 \\
5 \\
6 \\
7 \\
8 \\
9 \\
10 \\
11 \\
12 \\
13 \\
14 \\
15 \\
16 \\
17 \\
18 \\
19 \\
20 \\
21 \\
22 \\
23 \\
24 \\
25 \\
26 \\
27 \\
28 \\
29 \\
30 \\
31 \\
32 \\
33 \\
34 \\
35 \\
36 \\
37 \\
38 \\
39 \\
40 \\
41 \\
42 \\
43 \\
44 \\
45 \\
46 \\
47 \\
48 \\
49 \\
50 \\
51 \\
52 \\
53 \\
54 \\
55\end{array}$ & $\begin{array}{r}11 \\
11 \\
154 \\
171 \\
314 \\
324 \\
2 \\
4 \\
5 \\
7 \\
8 \\
10 \\
12 \\
23 \\
37 \\
50 \\
51 \\
65 \\
82 \\
98 \\
99 \\
116 \\
136 \\
153 \\
172 \\
189 \\
209 \\
226 \\
227 \\
243 \\
260 \\
274 \\
275 \\
288 \\
302 \\
313 \\
315 \\
317 \\
318 \\
320 \\
321 \\
323 \\
144 \\
15 \\
117 \\
18 \\
20 \\
21 \\
28 \\
29 \\
31 \\
32 \\
41 \\
43 \\
44\end{array}$ & $\begin{array}{l}C \\
C \\
C \\
C \\
C \\
C \\
F \\
F \\
F \\
F \\
F \\
F \\
F \\
F \\
F \\
F \\
F \\
F \\
F \\
F \\
F \\
F \\
F \\
F \\
F \\
F \\
F \\
F \\
F \\
F \\
F \\
F \\
F \\
F \\
F \\
F \\
F \\
F \\
F \\
F \\
F \\
F \\
R \\
R \\
R \\
R \\
R \\
R \\
R \\
R \\
R \\
R \\
R \\
R \\
R\end{array}$ & $\begin{array}{r}-3.70 \\
3.70 \\
-7.40 \\
7.40 \\
-3.70 \\
3.70 \\
-2.96 \\
-1.48 \\
-0.74 \\
0.74 \\
1.48 \\
2.96 \\
-4.07 \\
4.07 \\
-4.81 \\
4.81 \\
-5.18 \\
5.18 \\
-5.92 \\
5: 92 \\
-6.29 \\
6.29 \\
-7.03 \\
7.03 \\
-7.03 \\
7.03 \\
-6.29 \\
6.29 \\
-5.92 \\
5.92 \\
-5.18 \\
5.18 \\
-4.81 \\
4.81 \\
-4.07 \\
4.07 \\
-2.96 \\
-1.48 \\
-0.74 \\
0.74 \\
1.48 \\
2.96 \\
-2.59 \\
-1.85 \\
-0.37 \\
0.37 \\
1.85 \\
2.59 \\
-1.48 \\
-0.74 \\
0.74 \\
1.48 \\
-1.85 \\
-0.37 \\
0.37\end{array}$ & $\begin{array}{r}6.41 \\
6.41 \\
0.00 \\
0.00 \\
-6.41 \\
-6.41 \\
6.41 \\
6.41 \\
6.41 \\
6.41 \\
6.41 \\
6.41 \\
5.77 \\
5.77 \\
4.49 \\
4.49 \\
3.85 \\
3.85 \\
2.56 \\
2.56 \\
1.92 \\
1.92 \\
0.64 \\
0.64 \\
-0.64 \\
-0.64 \\
-1.92 \\
-1.92 \\
-2.56 \\
-2.56 \\
-3.85 \\
-3.85 \\
-4.49 \\
-4.49 \\
-5.77 \\
-5077 \\
-6.41 \\
-6.41 \\
-6.41 \\
-6.41 \\
-6.41 \\
-6.41 \\
5.77 \\
5.77 \\
5.77 \\
5.77 \\
5.77 \\
5.77 \\
5.13 \\
5.13 \\
5.13 \\
5.13 \\
4.49 \\
4.49 \\
4.49\end{array}$ & $\begin{array}{l}4 \cdot 2 \\
4: 2 \\
4: 2 \\
4: 2 \\
4: 2 \\
4: 2 \\
4: 3 \\
4: 3 \\
4: 3 \\
4: 4 \\
4: 3 \\
4: 2 \\
4: 2 \\
4: 2 \\
4: 4 \\
4: 2 \\
4: 4 \\
4: 2 \\
4: 4 \\
4: 2 \\
4: 4 \\
4: 2 \\
4: 2 \\
4: 2 \\
4: 2 \\
4: 2 \\
4: 3 \\
4: 2 \\
4: 3 \\
4.02 \\
4: 3 \\
4: 2 \\
4: 2 \\
4: 2 \\
4: 2 \\
4: 2 \\
4: 2 \\
4: 3 \\
4: 4 \\
4: 4 \\
4: 2 \\
4: 2 \\
4: 4 \\
4: 6 \\
4: 6 \\
4: 6 \\
4: 5 \\
4: 4 \\
4: 8 \\
4: 8 \\
4: 9 \\
4: 8 \\
5: 0 \\
4: 8 \\
5: 0\end{array}$ & $\begin{array}{l}12.8 \\
90.0 \\
10.2 \\
12.0 \\
13.6 \\
16.2 \\
15.0 \\
16.7 \\
16.6 \\
18.6 \\
16.7 \\
13.0 \\
13.1 \\
111.1 \\
17.0 \\
12.6 \\
16.5 \\
12.2 \\
19.7 \\
10.7 \\
17.3 \\
10.0 \\
13.0 \\
12.3 \\
12.2 \\
15.4 \\
15.0 \\
20.5 \\
16.0 \\
22.0 \\
24.8 \\
22.9 \\
21.5 \\
21.4 \\
16.5 \\
16.4 \\
16.0 \\
14.0 \\
17.3 \\
17.6 \\
21.0 \\
16.8 \\
18.4 \\
22.2 \\
22.3 \\
24.4 \\
21.4 \\
20.4 \\
20.0 \\
19.8 \\
21.1 \\
19.3 \\
21.9 \\
21.2 \\
22.5\end{array}$ & . & $\begin{array}{r}10.4693 \\
6.8888 \\
8.0008 \\
4.6750 \\
5.4627 \\
6.7831 \\
12.6589 \\
14.3662 \\
14.2648 \\
16.3626 \\
14.3662 \\
10.6625 \\
10.7593 \\
8.8461 \\
14.71140 \\
10.2765 \\
14.2051 \\
9.8924 \\
17.5139 \\
8.4692 \\
15.0208 \\
70.8142 \\
10.6625 \\
4.8212 \\
9.8924 \\
6.3715 \\
12.6589 \\
9.0765 \\
13.6590 \\
9.69088 \\
11.5399 \\
10.4161 \\
9.6295 \\
9.5739 \\
6.9386 \\
6.8867 \\
6.6797 \\
1116708 \\
15.0208 \\
15.3286 \\
9.3521 \\
7.0949 \\
16.15499 \\
20.2980 \\
2004069 \\
22.7219 \\
19.3763 \\
18.2540 \\
26.6911 \\
26.0680 \\
28.4977 \\
25.6025 \\
29.8525 \\
28.5773 \\
30.8169\end{array}$ & 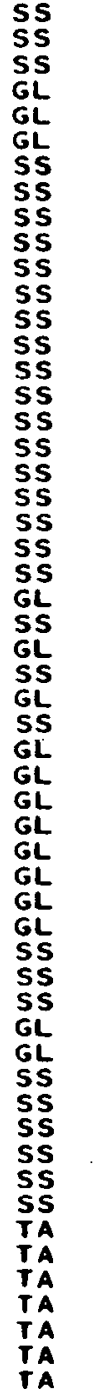 \\
\hline
\end{tabular}


Table B-2. (continued)

BLOCK 1-2926 (4.PSIG)

\begin{tabular}{|c|c|c|c|c|c|c|c|c|c|}
\hline OBS & HOLE & TYPF & $x A X I S$ & YAXIS & ME TER & MEASI & MEAS 2 & FLOW & GAGE \\
\hline $\begin{array}{l}56 \\
57 \\
58 \\
59 \\
60 \\
61 \\
62 \\
63 \\
64 \\
65 \\
66 \\
67 \\
68 \\
69 \\
70 \\
71 \\
72 \\
73 \\
74 \\
75 \\
76 \\
77 \\
78 \\
79 \\
80 \\
81 \\
82 \\
83 \\
84 \\
85 \\
86 \\
87 \\
88 \\
89 \\
90 \\
91 \\
92 \\
93 \\
94 \\
95 \\
96 \\
97 \\
98 \\
99 \\
100 \\
101 \\
102 \\
103 \\
104 \\
105 \\
106 \\
107 \\
108 \\
109 \\
110\end{array}$ & $\begin{array}{l}46 \\
56 \\
57 \\
59 \\
60 \\
71 \\
73 \\
74 \\
76 \\
88 \\
89 \\
91 \\
92 \\
156 \\
157 \\
159 \\
160 \\
165 \\
166 \\
168 \\
169 \\
173 \\
175 \\
176 \\
178 \\
183 \\
185 \\
186 \\
188 \\
191 \\
192 \\
194 \\
195 \\
203 \\
204 \\
206 \\
207 \\
211 \\
212 \\
214 \\
215 \\
220 \\
221 \\
223 \\
224 \\
228 \\
230 \\
231 \\
233 \\
237 \\
239 \\
240 \\
242 \\
245 \\
246\end{array}$ & $\begin{array}{l}R \\
R \\
R \\
R \\
R \\
R \\
R \\
R \\
R \\
R \\
R \\
R \\
R \\
R \\
R \\
R \\
R \\
R \\
R \\
R \\
R \\
R \\
R \\
R \\
R \\
R \\
R \\
R \\
R \\
R \\
R \\
R \\
R \\
R \\
R \\
R \\
R \\
R \\
R \\
R \\
R \\
R \\
R \\
R \\
R \\
R \\
R \\
R \\
R \\
R \\
R \\
R \\
R \\
R \\
R \\
R \\
R \\
R \\
R\end{array}$ & $\begin{array}{r}1.85 \\
-1.48 \\
-0.74 \\
0: 74 \\
1.48 \\
-1.85 \\
-0.37 \\
0.37 \\
1.85 \\
-1.48 \\
-0.74 \\
0.74 \\
1.48 \\
-5.92 \\
-5.18 \\
-3.70 \\
-2.96 \\
2.96 \\
3.70 \\
5.18 \\
5.92 \\
-6.29 \\
-4.81 \\
-4.07 \\
-2.59 \\
2.59 \\
4.07 \\
4.81 \\
6.29 \\
-5.92 \\
-5.18 \\
-3.70 \\
-2.96 \\
2.96 \\
3.70 \\
5.18 \\
5.92 \\
-4.81 \\
-4.07 \\
-2.59 \\
-11.85 \\
1.85 \\
2.59 \\
4.07 \\
4.81 \\
-5.18 \\
-3.70 \\
-2.96 \\
-1.48 \\
1.48 \\
2.96 \\
3.70 \\
5.18 \\
-4.81 \\
-4.07\end{array}$ & 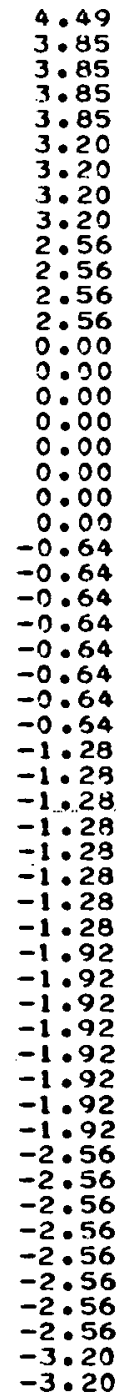 & $\begin{array}{l}5: 0 \\
5: 1 \\
5: 0 \\
5: 1 \\
5: 0 \\
5: 0 \\
5: 0 \\
5: 2 \\
5: 0 \\
5: 0 \\
5: 0 \\
5: 0 \\
5: 1 \\
4: 6 \\
4: 8 \\
5: 0 \\
5: 0 \\
5: 3 \\
5: 1 \\
4: 8 \\
4: 5 \\
4: 4 \\
4: 8 \\
5: 2 \\
5: 1 \\
5: 0 \\
5: 1 \\
4: 9 \\
4: 4 \\
4: 6 \\
4: 9 \\
5: 1 \\
5: 1 \\
5: 3 \\
5: 0 \\
4: 7 \\
4: 4 \\
4: 8 \\
5: 1 \\
5: 0 \\
5: 2 \\
5: 3 \\
4: 9 \\
4: 9 \\
4: 8 \\
4: 7 \\
5: 0 \\
5: 1 \\
5: 2 \\
5: 2 \\
5: 1 \\
4: 9 \\
4: 5 \\
4: 5 \\
4: 9\end{array}$ & 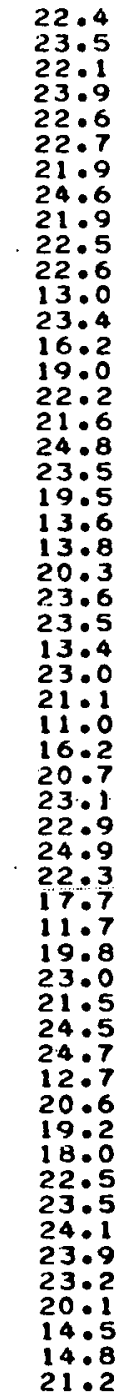 & 15.4 & 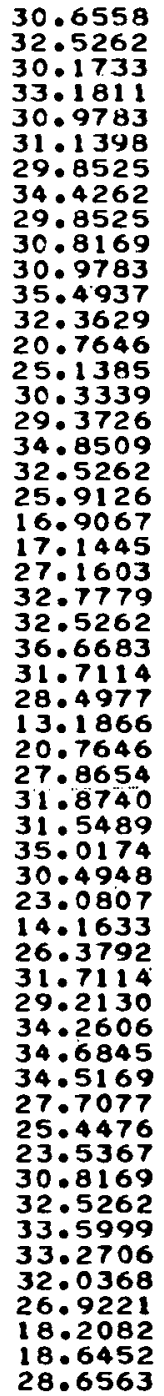 & 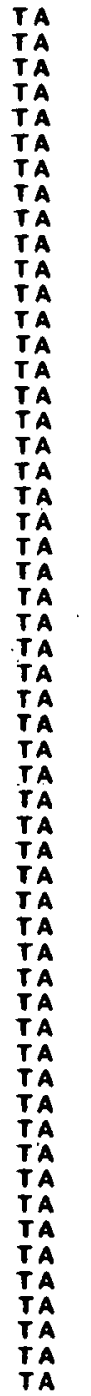 \\
\hline
\end{tabular}


Table B-2. (continued)

ELOCK 1-2926 (4 PSIG)

\begin{tabular}{|c|c|c|c|c|c|c|c|c|c|}
\hline OBS & HOLE & TYPE & XAXIS & YAXIS & METER & MEAS 1 & MEAS 2 & FLOW & GAGE \\
\hline 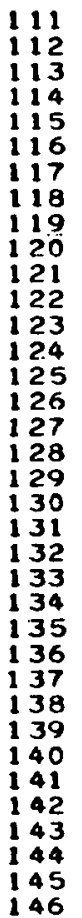 & $\begin{array}{l}248 \\
249 \\
254 \\
255 \\
257 \\
258 \\
262 \\
263 \\
271 \\
272 \\
276 \\
278 \\
285 \\
287 \\
290 \\
291 \\
299 \\
300 \\
107 \\
108 \\
124 \\
128 \\
143 \\
146 \\
179 \\
182 \\
197 \\
201 \\
217 \\
218 \\
125 \\
127 \\
162 \\
163 \\
198 \\
200\end{array}$ & $\begin{array}{l}R \\
R \\
R \\
R \\
R \\
R \\
R \\
R \\
R \\
R \\
R \\
R \\
R \\
R \\
R \\
R \\
R \\
R \\
S \\
S \\
S \\
S \\
S \\
S \\
S \\
S \\
S \\
S \\
S \\
S \\
I \\
I \\
I \\
I \\
I \\
I\end{array}$ & 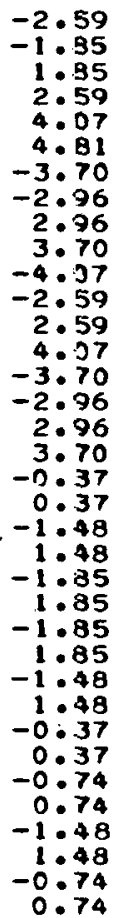 & $\begin{array}{r}-3 \cdot 20 \\
-3.20 \\
-3.20 \\
-3.20 \\
-3.20 \\
-3.20 \\
-3.85 \\
-3.85 \\
-3.85 \\
-3.85 \\
-4.49 \\
-4.49 \\
-4.49 \\
-4.86 \\
-5.13 \\
-5.13 \\
-5.13 \\
-5.13 \\
1.92 \\
1.92 \\
1.28 \\
1.28 \\
0.64 \\
0.64 \\
-0.64 \\
-0.64 \\
-1.28 \\
-1.28 \\
-1.922 \\
-1.92 \\
1.28 \\
1.28 \\
0.000 \\
0.00 \\
-1.28 \\
-1.28\end{array}$ & $\begin{array}{l}4 \cdot 9 \\
5: 1 \\
5: 1 \\
5: 1 \\
4: 7 \\
4 \cdot 5 \\
4: 9 \\
4: 8 \\
5: 0 \\
4: 6 \\
4: 5 \\
4: 7 \\
4: 8 \\
4 \cdot 4 \\
4: 4 \\
4: 5 \\
4: 4 \\
4: 4 \\
5: c \\
4: 9 \\
5: 0 \\
5: 2 \\
5: 0 \\
5: 2 \\
5: 0 \\
5: 0 \\
5: 2 \\
5: 0 \\
5: 0 \\
5: 1 \\
4: 8 \\
5: 2 \\
4: 8 \\
5: 1 \\
5: 1 \\
5.0\end{array}$ & $\begin{array}{l}20.7 \\
23.0 \\
23.0 \\
23.4 \\
18.5 \\
15.3 \\
21.0 \\
21: 0 \\
21.4 \\
17.3 \\
14.1 \\
18.2 \\
19.9 \\
12.0 \\
12.3 \\
14.3 \\
13.6 \\
10.1 \\
22.7 \\
12.9 \\
22.6 \\
24.5 \\
22.9 \\
24: 1 \\
13.0 \\
13.0 \\
24.6 \\
13.0 \\
13.2 \\
13.8 \\
20.0 \\
24.0 \\
20.1 \\
22.9 \\
23.7 \\
13.5\end{array}$ & $\begin{array}{l}15.0 \\
15.0 \\
15.0 \\
15.2 \\
16.2\end{array}$ & $\begin{array}{l}27.8654 \\
31.7114 \\
31.7114 \\
32.3629 \\
24.3002 \\
19.3770 \\
28.3394 \\
28.2612 \\
29.0536 \\
22.4114 \\
17.6280 \\
23.8416 \\
26.5351 \\
14.5845 \\
15.0072 \\
17.9178 \\
16.8573 \\
11.9434 \\
31.1398 \\
34.9522 \\
30.9783 \\
34.2606 \\
31.4634 \\
33.5999 \\
35.4937 \\
35.4937 \\
34.4262 \\
35.4937 \\
36.0803 \\
38.5579 \\
26.6911 \\
33.4351 \\
26.8474 \\
31.5489 \\
32.8533 \\
36.9628\end{array}$ & $\begin{array}{l}\text { TA } \\
\text { TAA } \\
\text { TAA } \\
\text { TA } \\
\text { TA } \\
\text { TA } \\
\text { TAA } \\
\text { TA } \\
\text { TA } \\
\text { TA } \\
\text { TA } \\
\text { TA } \\
\text { TA } \\
\text { TA } \\
\text { TA } \\
\text { TA } \\
\text { IA } \\
\text { TA } \\
\text { TA } \\
\text { TA } \\
\text { TA } \\
\text { TA } \\
\text { TA } \\
\text { TA } \\
\text { TA } \\
\text { TA } \\
\text { TA } \\
\text { TA } \\
\text { TA } \\
\text { TA } \\
\text { TA } \\
\text { TA } \\
\text { TA }\end{array}$ \\
\hline
\end{tabular}


Table B-2. (continued)

BLOCK 1-4748 (4 PSIG)

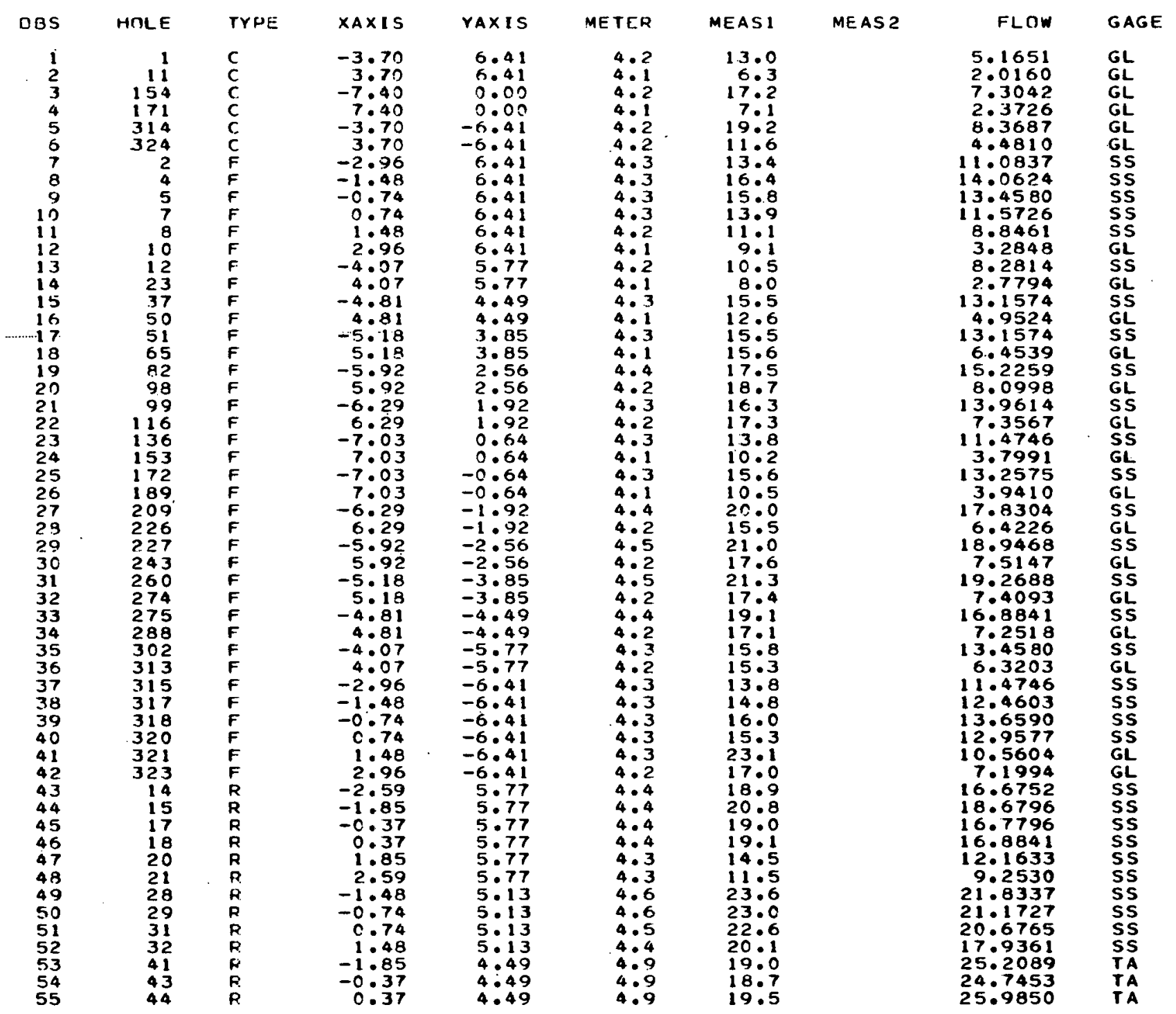


Table B-2. (continued)

9:-OCK $1-4748$ (4 PSIG).

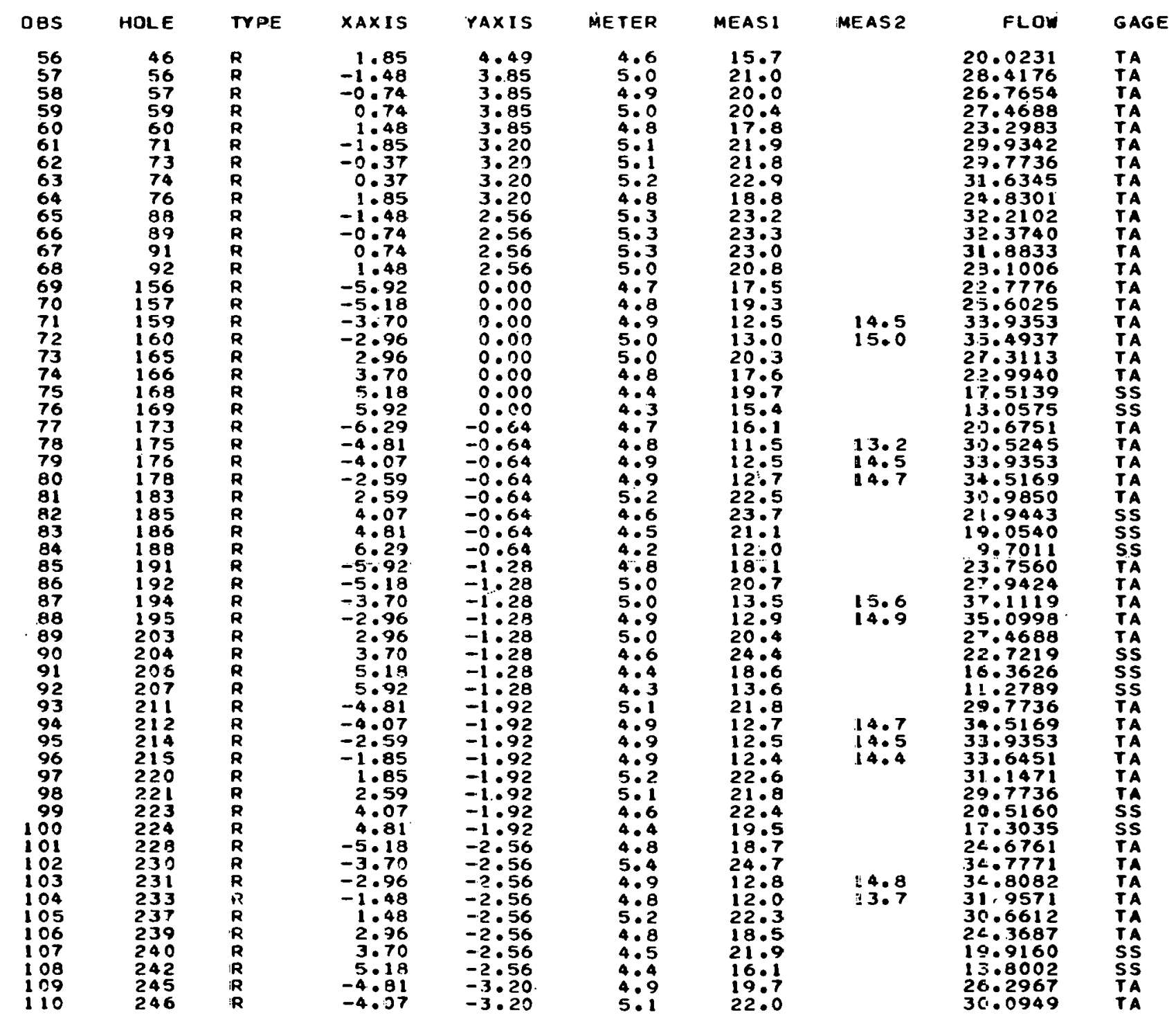


Table B-2. (continued)

PLOCK 1-4748 (4 PSIG)

\begin{tabular}{|c|c|c|c|c|c|c|c|c|c|}
\hline OBS & HOLE & TYPE & XAXIS & YAXIS & METER & MEASI & MEAS 2 & FLOW & GAG \\
\hline $\begin{array}{lll}1 & 1 & 1 \\
1 & 1 & 2 \\
1 & 1 & 3 \\
1 & 1 & 4 \\
1 & 1 & 5 \\
1 & 1 & 6 \\
1 & 1 & 7 \\
1 & 1 & 3 \\
1 & 1 & 7 \\
1 & 25 \\
1 & 21 \\
1 & 22 \\
1 & 23 \\
1 & 24 \\
1 & 25 \\
1 & 26 \\
1 & 27 \\
1 & 28 \\
1 & 29 \\
1 & 30 \\
1 & 31 \\
1 & 32 \\
1 & 33 \\
1 & 34 \\
1 & 35 \\
1 & 36 \\
1 & 37 \\
1 & 38 \\
1 & 39 \\
1 & 40 \\
1 & 41 \\
1 & 42 \\
1 & 4.3 \\
1 & 44 \\
1 & 45 \\
1 & 46\end{array}$ & $\begin{array}{l}248 \\
249 \\
254 \\
255 \\
257 \\
258 \\
262 \\
263 \\
271 \\
272 \\
276 \\
278 \\
285 \\
287 \\
290 \\
291 \\
299 \\
300 \\
107 \\
108 \\
124 \\
128 \\
143 \\
146 \\
179 \\
182 \\
197 \\
201 \\
217 \\
218 \\
125 \\
127 \\
162 \\
163 \\
198 \\
200\end{array}$ & $\begin{array}{l}R \\
R \\
R \\
R \\
R \\
R \\
R \\
R \\
R \\
R \\
R \\
R \\
R \\
R \\
R \\
R \\
R \\
R \\
S \\
S \\
S \\
S \\
S \\
S \\
S \\
S \\
S \\
S \\
S \\
S \\
I \\
I \\
I \\
I \\
I \\
I\end{array}$ & $\begin{array}{r}-2.59 \\
-1.85 \\
1.85 \\
2.59 \\
4.07 \\
4.81 \\
-3.70 \\
-2.96 \\
2.96 \\
3.70 \\
-4.07 \\
-2.59 \\
2.59 \\
4.07 \\
-3.70 \\
-2.96 \\
2.96 \\
3.70 \\
-0.37 \\
0.37 \\
-1.48 \\
1.48 \\
-1.85 \\
1.85 \\
-1.85 \\
1.85 \\
-1.48 \\
1.48 \\
-0.37 \\
0.37 \\
-0.74 \\
0.74 \\
-1.48 \\
-1.48 \\
-0.74 \\
0.74\end{array}$ & $\begin{array}{r}-3.20 \\
-3.20 \\
-3.20 \\
-3.20 \\
-3.20 \\
-3.20 \\
-3.85 \\
-3.85 \\
-3.85 \\
-3.85 \\
-4.49 \\
-4.49 \\
-4.49 \\
-4.86 \\
-5.13 \\
-5.13 \\
-5.13 \\
-5.13 \\
1.922 \\
1.92 \\
1.28 \\
1.28 \\
0.64 \\
0.64 \\
-0.64 \\
-0.64 \\
-1.28 \\
-1.28 \\
-1.92 \\
-1.92 \\
1.28 \\
1.28 \\
0.00 \\
0.00 \\
-1.28 \\
-1.28\end{array}$ & $\begin{array}{l}5: 4 \\
4: 9 \\
4: 9 \\
4: 8 \\
4: 4 \\
4: 4 \\
5: 0 \\
5: ? \\
4: 5 \\
4: 4 \\
4: 9 \\
5: 0 \\
4: 5 \\
4: 3 \\
4: 7 \\
4: 8 \\
4: 4 \\
4: 3 \\
5: 2 \\
5: 2 \\
5: 3 \\
5: 1 \\
5: 0 \\
5: 1 \\
5: 0 \\
5: 3 \\
5: 2 \\
5: 3 \\
5: 1 \\
4: 9 \\
5: 0 \\
5: 0 \\
5: 2 \\
5: 0 \\
5: 2 \\
5.1\end{array}$ & $\begin{array}{l}24.5 \\
12.2 \\
20.0 \\
17.7 \\
19.7 \\
16.5 \\
21.1 \\
22.6 \\
22.0 \\
18.8 \\
17.9 \\
21.1 \\
21.2 \\
14.2 \\
17.2 \\
18.0 \\
17.0 \\
13.6 \\
23.8 \\
23.9 \\
24.2 \\
22.7 \\
13.1 \\
22.6 \\
12.9 \\
24.6 \\
24.2 \\
24.6 \\
23.6 \\
12.6 \\
22.1 \\
22.3 \\
23.7 \\
22.3 \\
23.6 \\
23.3\end{array}$ & $\begin{array}{l}15.1 \\
14.9\end{array}$ & $\begin{array}{l}34.4442 \\
32.7731 \\
26.7654 \\
23.1461 \\
17.5139 \\
14.2051 \\
28.5763 \\
31.1471 \\
20.0242 \\
16.5709 \\
23.4507 \\
28.5763 \\
19.1613 \\
11.8675 \\
22.3242 \\
23.6032 \\
14.7140 \\
11.2799 \\
33.1062 \\
33.2706 \\
33.8554 \\
31.2246 \\
35.77868 \\
31.0627 \\
35.2009 \\
34.5184 \\
33.7648 \\
34.5184 \\
32.6896 \\
34.2259 \\
30.1733 \\
30.44948 \\
32.9420 \\
30.4948 \\
32.7779 \\
32.1997\end{array}$ & $\begin{array}{l}\text { TA } \\
\text { TA } \\
\text { TA } \\
\text { TA } \\
\text { SS } \\
\text { SS } \\
\text { TA } \\
\text { TA } \\
\text { SS } \\
\text { SS } \\
\text { TA } \\
\text { TA } \\
\text { SS } \\
\text { SS } \\
\text { TA } \\
\text { TA } \\
\text { SS } \\
\text { SS } \\
\text { TA } \\
\text { TA } \\
\text { TA } \\
\text { TA } \\
\text { TA } \\
\text { TA } \\
\text { TA } \\
\text { TA } \\
\text { TA } \\
\text { TA } \\
\text { TA } \\
\text { TA } \\
\text { TA } \\
\text { TA } \\
\text { TA } \\
\text { TA } \\
\text { TA }\end{array}$ \\
\hline
\end{tabular}


Table B-2. (continued)

BLOCK 1-4749 (4 PSEG)

\begin{tabular}{|c|c|c|c|c|c|c|c|c|c|}
\hline oBs & HOLE & TYPE & XAXIS & YAXIS & METER & MEASI & MEAS 2 & FLOW & GAGE \\
\hline $\begin{array}{l}1 \\
2 \\
3 \\
4 \\
5 \\
6 \\
7 \\
8 \\
9 \\
10 \\
11 \\
12 \\
13 \\
14 \\
15 \\
16 \\
17 \\
18 \\
199 \\
20 \\
21 \\
22 \\
23 \\
24 \\
25 \\
26 \\
27 \\
28 \\
29 \\
30 \\
31 \\
32 \\
33 \\
34 \\
35 \\
36 \\
37 \\
38 \\
39 \\
40 \\
41 \\
42 \\
43 \\
44 \\
45 \\
46 \\
47 \\
48 \\
499 \\
50 \\
51 \\
52 \\
53 \\
54 \\
55\end{array}$ & $\begin{array}{r}11 \\
11 \\
154 \\
171 \\
314 \\
324 \\
2 \\
4 \\
5 \\
7 \\
8 \\
110 \\
12 \\
23 \\
37 \\
50 \\
51 \\
65 \\
82 \\
98 \\
99 \\
116 \\
136 \\
153 \\
172 \\
189 \\
209 \\
226 \\
227 \\
243 \\
260 \\
274 \\
275 \\
288 \\
322 \\
313 \\
315 \\
317 \\
318 \\
320 \\
321 \\
323 \\
144 \\
15 \\
117 \\
118 \\
20 \\
21 \\
288 \\
29 \\
31 \\
32 \\
41 \\
43 \\
44\end{array}$ & $\begin{array}{l}C \\
C \\
C \\
C \\
C \\
C \\
F \\
F \\
F \\
F \\
F \\
F \\
F \\
F \\
F \\
F \\
F \\
F \\
F \\
F \\
F \\
F \\
F \\
F \\
F \\
F \\
F \\
F \\
F \\
F \\
F \\
F \\
F \\
F \\
F \\
F \\
F \\
F \\
F \\
F \\
F \\
F \\
R \\
R \\
R \\
R \\
R \\
R \\
R \\
R \\
R \\
R \\
R \\
R \\
R\end{array}$ & $\begin{array}{r}-3.70 \\
3.70 \\
-7.40 \\
7.40 \\
-3.70 \\
3.70 \\
-2.96 \\
-1.48 \\
-0.74 \\
0.74 \\
1.48 \\
2.96 \\
-4.07 \\
4.07 \\
-4.81 \\
4.81 \\
-5.18 \\
5.18 \\
-5.92 \\
5.92 \\
-6.29 \\
6.29 \\
-7.03 \\
7.03 \\
-7.03 \\
7.03 \\
-6.29 \\
6.29 \\
-5.92 \\
5.92 \\
-5.18 \\
5.18 \\
-4.81 \\
4.81 \\
-4.07 \\
4.07 \\
-2.96 \\
-1.48 \\
-0.74 \\
0.74 \\
1.48 \\
2.96 \\
-2.59 \\
-1.85 \\
-0.37 \\
0.37 \\
1.085 \\
2.59 \\
-1.48 \\
-0.74 \\
0.74 \\
1.48 \\
-1.83 \\
-0.37 \\
0.37\end{array}$ & $\begin{array}{r}6.41 \\
6.41 \\
0.000 \\
0.00 \\
-6.41 \\
-6.41 \\
6.41 \\
6.41 \\
6.41 \\
6.41 \\
6.41 \\
6.41 \\
5.77 \\
5.77 \\
4.49 \\
4.49 \\
3.85 \\
3.85 \\
2.56 \\
2.56 \\
1.92 \\
1.92 \\
0.64 \\
0.64 \\
-0.64 \\
-0.64 \\
-1.92 \\
-1.92 \\
-2.56 \\
-2.56 \\
-3.85 \\
-3.85 \\
-4.49 \\
-4.49 \\
-5.77 \\
-5.77 \\
96.41 \\
-6.41 \\
-6.41 \\
-6.41 \\
-6.41 \\
-6.41 \\
5.77 \\
5.77 \\
5.77 \\
5.77 \\
5.77 \\
5.77 \\
5.13 \\
5.13 \\
5.13 \\
5.13 \\
4.49 \\
4.49 \\
4.49\end{array}$ & $\begin{array}{l}4 \cdot 1 \\
4: 1 \\
4: 1 \\
4: 2 \\
4: 2 \\
4: 1 \\
4: 1 \\
4: 2 \\
4: 2 \\
4: 2 \\
4: 2 \\
4: 2 \\
4: 1 \\
4: 2 \\
4: 2 \\
4: 3 \\
4: 2 \\
4: 3 \\
4: 2 \\
4: 3 \\
4: 2 \\
4: 2 \\
4: 1 \\
4: 2 \\
4: 1 \\
4: 2 \\
4: 2 \\
4: 4 \\
4: 2 \\
4: 4 \\
4: 2 \\
4: 4 \\
4: 2 \\
4: 4 \\
4: 2 \\
4: 2 \\
4: 2 \\
4: 4 \\
4: 4 \\
4: 3 \\
4: 2 \\
4: 2 \\
4: 2 \\
4: 3 \\
4: 4 \\
4: 5 \\
4: 4 \\
4: 4 \\
4: 5 \\
4: 6 \\
4: 7 \\
4: 6 \\
4: 5 \\
5: 8 \\
: 2 \\
: 5\end{array}$ & $\begin{array}{r}5.6 \\
10.6 \\
6.5 \\
13.0 \\
13.0 \\
7.5 \\
8.5 \\
15.6 \\
18.5 \\
21.8 \\
21.4 \\
16.2 \\
7.5 \\
16.4 \\
12.5 \\
24.5 \\
14.2 \\
24.9 \\
13.6 \\
23.7 \\
11.5 \\
22.0 \\
8.3 \\
18.1 \\
8.4 \\
20.4 \\
7.8 \\
16.5 \\
10.3 \\
17.6 \\
13.0 \\
19.8 \\
11.5 \\
18.2 \\
10.0 \\
9.0 \\
11.4 \\
17.0 \\
18.2 \\
14.6 \\
21.0 \\
11.0 \\
18.2 \\
25.0 \\
19.4 \\
20.7 \\
18.3 \\
17.0 \\
20.8 \\
22.6 \\
25.0 \\
23.1 \\
22.5 \\
18.3 \\
20: 3\end{array}$ & & $\begin{array}{r}1.7079 \\
3.9884 \\
2.1047 \\
5.1651 \\
5.1651 \\
2.5527 \\
3.0080 \\
6.4739 \\
7.9928 \\
9.7969 \\
9.5739 \\
6.7831 \\
5.5057 \\
6.8867 \\
10.11803 \\
11.3655 \\
11.8320 \\
11.5982 \\
11.2451 \\
10.9037 \\
9.2249 \\
9.9088 \\
6.2286 \\
7.7796 \\
6.3195 \\
9.0217 \\
5.7942 \\
14.2051 \\
8.0942 \\
15.3286 \\
10.6625 \\
17.6192 \\
9.2249 \\
15.9476 \\
7.8142 \\
6.8889 \\
9.1300 \\
14.7140 \\
15.9476 \\
12.2622 \\
9.3521 \\
4.1923 \\
7.8328 \\
11.6566 \\
17.1985 \\
18.6259 \\
16.0512 \\
14.7140 \\
18.7327 \\
20.7344 \\
23.4573 \\
21.2826 \\
20.5675 \\
24.0620 \\
27.3113\end{array}$ & 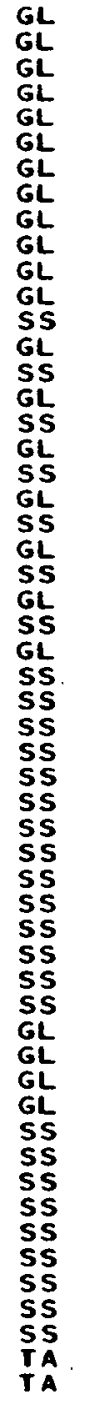 \\
\hline
\end{tabular}


Table B-2. (continued)

BLOCK 1-4749 (4 PSIG)

\begin{tabular}{|c|c|c|c|c|c|c|c|c|c|}
\hline OHS & HDLE & TYPE & $X A X I S$ & YAXIS & ME TER & MEASI & MEAS 2 & FLOW & GAGE \\
\hline $\begin{array}{l}56 \\
57 \\
58 \\
59 \\
60 \\
61 \\
62 \\
63 \\
64 \\
65 \\
66 \\
67 \\
68 \\
69 \\
70 \\
71 \\
72 \\
73 \\
74 \\
75 \\
76 \\
77 \\
78 \\
79 \\
80 \\
81 \\
82 \\
83 \\
84 \\
85 \\
86 \\
87 \\
88 \\
89 \\
90 \\
91 \\
92 \\
93 \\
94 \\
95 \\
96 \\
97 \\
98 \\
99 \\
100 \\
101 \\
102 \\
103 \\
104 \\
105 \\
106 \\
107 \\
108 \\
109 \\
110\end{array}$ & 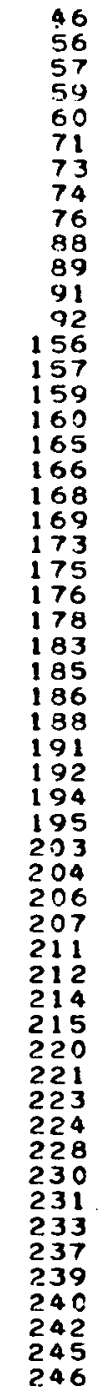 & $\begin{array}{l}R \\
R \\
R \\
R \\
R \\
R \\
R \\
R \\
R \\
R \\
R \\
R \\
R \\
R \\
R \\
R \\
R \\
R \\
R \\
R \\
R \\
R \\
R \\
R \\
R \\
R \\
R \\
R \\
R \\
R \\
R \\
R \\
R \\
R \\
R \\
R \\
R \\
R \\
R \\
R \\
R \\
R \\
R \\
R \\
R \\
R \\
R \\
R \\
R \\
R \\
R \\
R \\
R \\
R \\
R \\
R \\
R \\
R \\
R \\
R\end{array}$ & $\begin{array}{r}1.85 \\
-1.48 \\
-1.74 \\
0.74 \\
1.48 \\
-1.85 \\
-0.37 \\
0.37 \\
1.85 \\
-1.49 \\
-0.74 \\
0.74 \\
1.48 \\
-5.92 \\
-5.18 \\
-3.70 \\
-2.96 \\
2.96 \\
3.70 \\
5.18 \\
5.92 \\
-6.29 \\
-4.81 \\
-4.07 \\
-2.59 \\
2.59 \\
4.07 \\
4.81 \\
6.29 \\
-5.92 \\
-5.19 \\
-3.70 \\
-2.96 \\
2.96 \\
3.70 \\
5.18 \\
5.92 \\
-4.81 \\
-4.07 \\
-2.59 \\
-1.85 \\
1.85 \\
2.59 \\
4.07 \\
4.81 \\
-5.118 \\
-3.70 \\
-2.96 \\
-1.48 \\
1.48 \\
2.96 \\
3.70 \\
5.19 \\
-4.81 \\
-4.07\end{array}$ & $\begin{array}{r}4.49 \\
3.85 \\
3.85 \\
3.85 \\
3.85 \\
3.20 \\
3.20 \\
3.20 \\
3.20 \\
2.56 \\
2.56 \\
2.56 \\
2.56 \\
0.00 \\
0.00 \\
0.00 \\
0.000 \\
0.00 \\
0.00 \\
0.00 \\
0.00 \\
-0.64 \\
-0.64 \\
-0.64 \\
-0.64 \\
-0.64 \\
-0.64 \\
-0.64 \\
-0.64 \\
-1.28 \\
-1.28 \\
-1.28 \\
-1.28 \\
-1.28 \\
-1.28 \\
-1.28 \\
-1.28 \\
-1.92 \\
-1.92 \\
-1.92 \\
-1.92 \\
-1.92 \\
-1.92 \\
-1.92 \\
-1.92 \\
-2.56 \\
-2.56 \\
-2.56 \\
-2.56 \\
-2.56 \\
-2.56 \\
-2.56 \\
-2.56 \\
-3.20 \\
-3.20\end{array}$ & $\begin{array}{l}4: 3 \\
4: y \\
5: 0 \\
5: 0 \\
5: 0 \\
4: 9 \\
5: 2 \\
5: 3 \\
5: 1 \\
5: 3 \\
5: 2 \\
5: 3 \\
5: 2 \\
4: 2 \\
4: 4 \\
4: 8 \\
4: 9 \\
5: 4 \\
5: 3 \\
4: 9 \\
4: 6 \\
4: 2 \\
4: 4 \\
4: 7 \\
5: 1 \\
5: 0 \\
5: 1 \\
5: 0 \\
4: 5 \\
4: 2 \\
4: 44 \\
4: 8 \\
4: 9 \\
5: 0 \\
5: 2 \\
4: 9 \\
4: 6 \\
4: 44 \\
4: 7 \\
5: 9 \\
5: 0 \\
5: 0 \\
5: 1 \\
5: 0 \\
4: 44 \\
4: 7 \\
5: 8 \\
5: 0 \\
5: 0 \\
5: 1 \\
4: 8 \\
4: 4 \\
4: 5\end{array}$ & $\begin{array}{l}17.6 \\
18.9 \\
20.0 \\
20.8 \\
211.1 \\
19.8 \\
22.1 \\
23.4 \\
21.9 \\
23.0 \\
22.4 \\
23.6 \\
23.0 \\
71.5 \\
11.3 \\
18.9 \\
19.8 \\
13.1 \\
23.4 \\
19.1 \\
15.6 \\
6.3 \\
12.9 \\
16.9 \\
21.4 \\
12.8 \\
22.0 \\
20.4 \\
14.0 \\
8.0 \\
11.8 \\
17.7 \\
19.7 \\
12.6 \\
22.8 \\
19.1 \\
15.4 \\
12.0 \\
16.0 \\
19.5 \\
22.0 \\
12.6 \\
13.4 \\
22.2 \\
20.0 \\
10.6 \\
16.5 \\
17.5 \\
211.7 \\
12.6 \\
12.7 \\
22.2 \\
17.6 \\
11.4 \\
13.5\end{array}$ & $\begin{array}{l}15.1 \\
14.8\end{array}$ & $\begin{array}{l}22.9940 \\
25.0542 \\
26.8397 \\
28.1006 \\
28.5763 \\
26.4527 \\
30.3382 \\
32.5379 \\
29.9342 \\
31.8833 \\
30.8230 \\
32.8662 \\
31.7973 \\
8.3797 \\
13.6041 \\
24.9842 \\
26.4527 \\
36.1975 \\
32.5379 \\
25.3638 \\
19.8753 \\
6.8076 \\
15.8574 \\
21.8723 \\
29.1333 \\
34.9085 \\
30.0949 \\
27.4688 \\
17.4834 \\
9.0421 \\
14.3035 \\
23.1461 \\
26.2967 \\
34.3247 \\
31.4719 \\
25.3638 \\
19.5802 \\
14.5845 \\
20.5262 \\
2509850 \\
30.1769 \\
34.3247 \\
36.6683 \\
30.4168 \\
26.8397 \\
12.6323 \\
21.2723 \\
22.8421 \\
29.6133 \\
34.3247 \\
34.6164 \\
30.4168 \\
22.9940 \\
13.7436 \\
16.7629\end{array}$ & 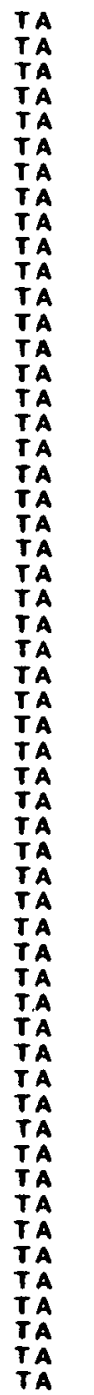 \\
\hline
\end{tabular}


Table B-2. (continued)

BLOCK I-4749 (4 PSIG)

\begin{tabular}{|c|c|c|c|c|c|c|c|c|c|}
\hline oBs & HOLE & TYPE & XAXIS & YAXIS & MEṬER & MEASI & MEAS 2 & · FLOW & GAGE \\
\hline 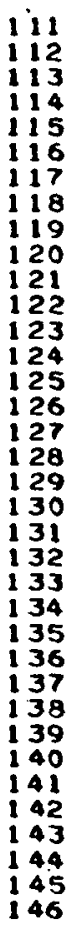 & $\begin{array}{l}248 \\
249 \\
254 \\
255 \\
257 \\
258 \\
262 \\
263 \\
271 \\
272 \\
276 \\
278 \\
285 \\
287 \\
290 \\
291 \\
299 \\
300 \\
107 \\
108 \\
124 \\
128 \\
143 \\
146 \\
179 \\
182 \\
197 \\
201 \\
217 \\
218 \\
125 \\
127 \\
162 \\
163 \\
198 \\
200\end{array}$ & $\begin{array}{l}R \\
R \\
R \\
R \\
R \\
R \\
R \\
R \\
R \\
R \\
R \\
R \\
R \\
R \\
R \\
R \\
R \\
R \\
S \\
S \\
S \\
S \\
S \\
S \\
S \\
S \\
S \\
S \\
S \\
S \\
I \\
I \\
I \\
I \\
I \\
I\end{array}$ & $\begin{array}{r}-2.59 \\
-1.85 \\
1.85 \\
2.59 \\
4.07 \\
4.81 \\
-3.70 \\
-2.96 \\
2.96 \\
3.70 \\
-4.07 \\
-2.59 \\
2.59 \\
4.07 \\
-3.70 \\
-2.96 \\
2.96 \\
3.70 \\
-0.37 \\
0.37 \\
-1.48 \\
1.48 \\
-1.85 \\
1.85 \\
-1.85 \\
1.85 \\
-1.48 \\
1.448 \\
-0.37 \\
0.37 \\
-0.74 \\
0.74 \\
-1.48 \\
1.48 \\
-0.74 \\
0.74\end{array}$ & $\begin{array}{r}-3 \cdot 20 \\
-3.20 \\
-3.20 \\
-3.20 \\
-3.20 \\
-3.20 \\
-3.85 \\
-3.85 \\
-3.85 \\
-3.85 \\
-4.49 \\
-4.49 \\
-4.49 \\
-4.86 \\
-5.13 \\
-5.13 \\
-5: 13 \\
-5.13 \\
1.92 \\
1: 92 \\
1.28 \\
1.28 \\
0.64 \\
0.64 \\
-0.64 \\
-0.64 \\
-1.28 \\
-1.28 \\
-1.92 \\
-1.92 \\
1.28 \\
1.228 \\
0.00 \\
0.00 \\
-1.28 \\
-1.28\end{array}$ & $\begin{array}{l}4.8 \\
5: 0 \\
5: 4 \\
5: 0 \\
5: 0 \\
4: 8 \\
4: 6 \\
4: 6 \\
5: 2 \\
4: 9 \\
4: 4 \\
4: 6 \\
5.0 \\
4: 6 \\
4: 4 \\
4: 5 \\
4: 6 \\
4: 4 \\
5: 0 \\
5: 2 \\
5: 0 \\
5: 2 \\
5: 2 \\
5: 2 \\
5: 1 \\
5: 2 \\
5: 0 \\
5: 2 \\
5: 0 \\
5: 2 \\
4: 9 \\
5: 0 \\
5: 0 \\
5: 0 \\
5: 0 \\
5.0\end{array}$ & $\begin{array}{l}18.4 \\
20.3 \\
24.9 \\
12: 6 \\
20: 6 \\
18.1 \\
14.9 \\
15.4 \\
22.5 \\
19.6 \\
11.5 \\
15.4 \\
20.4 \\
15.6 \\
10.8 \\
13.4 \\
15.1 \\
12.6 \\
21.6 \\
23.5 \\
21.6 \\
23.0 \\
22.6 \\
23.0 \\
21.9 \\
23.1 \\
21.6 \\
22.5 \\
21.4 \\
22.3 \\
20: 3 \\
20.9 \\
20.9 \\
211.4 \\
21.6 \\
20.6\end{array}$ & $\begin{array}{l}14.5 \\
.\end{array}$ & $\begin{array}{l}24.2153 \\
27.3113 \\
35.1108 \\
34.1773 \\
27.7843 \\
23.7560 \\
18.8456 \\
19.5802 \\
30.9850 \\
26.1407 \\
13.8833 \\
19.5802 \\
27.4688 \\
19.8753 \\
12.9091 \\
16.6194 \\
19.1390 \\
1594316 \\
29.3726 \\
32.6141 \\
29.3726 \\
31.7973 \\
31.1471 \\
31.7973 \\
29.9342 \\
31.0603 \\
29.3726 \\
30.9850 \\
29.0536 \\
30.6612 \\
27.2358 \\
28.2590 \\
28.0590 \\
29.0536 \\
29.3726 \\
27.7843\end{array}$ & 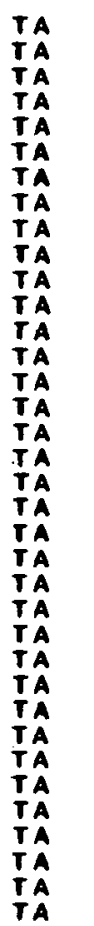 \\
\hline
\end{tabular}


Table B-2. (continued)

BLOCK 1-5285 (4 PSIG)

\begin{tabular}{|c|c|c|c|c|c|c|c|c|c|}
\hline a9s & HOLE & TYPE & XAXIS & YAXIS & METER & MEAS1 & MEAS 2 & FLOW & GAGE \\
\hline $\begin{array}{l}1 \\
2 \\
3 \\
4 \\
5 \\
6 \\
7 \\
8 \\
9 \\
10 \\
11 \\
12 \\
13 \\
14 \\
15 \\
16 \\
17 \\
18 \\
19 \\
20 \\
21 \\
22 \\
23 \\
24 \\
25 \\
26 \\
27 \\
28 \\
29 \\
30 \\
31 \\
32 \\
33 \\
34 \\
35 \\
36 \\
37 \\
38 \\
39 \\
40 \\
41 \\
42 \\
43 \\
44 \\
45 \\
46 \\
47 \\
48 \\
49 \\
50 \\
51 \\
52 \\
53 \\
54 \\
55\end{array}$ & $\begin{array}{r}1 \\
11 \\
154 \\
171 \\
314 \\
324 \\
2 \\
4 \\
5 \\
7 \\
8 \\
10 \\
12 \\
23 \\
37 \\
50 \\
51 \\
65 \\
82 \\
98 \\
99 \\
116 \\
136 \\
153 \\
172 \\
199 \\
209 \\
226 \\
227 \\
243 \\
260 \\
274 \\
275 \\
288 \\
302 \\
313 \\
315 \\
317 \\
318 \\
320 \\
321 \\
323 \\
144 \\
15 \\
17 \\
188 \\
20 \\
21 \\
28 \\
29 \\
31 \\
32 \\
41 \\
43 \\
44\end{array}$ & $\begin{array}{l}C \\
C \\
C \\
C \\
C \\
C \\
F \\
F \\
F \\
F \\
F \\
F \\
F \\
F \\
F \\
F \\
F \\
F \\
F \\
F \\
F \\
F \\
F \\
F \\
F \\
F \\
F \\
F \\
F \\
F \\
F \\
F \\
F \\
F \\
F \\
F \\
F \\
F \\
F \\
F \\
F \\
F \\
R \\
R \\
R \\
R \\
R \\
R \\
R \\
R \\
R \\
R \\
R \\
R \\
R\end{array}$ & $\begin{array}{r}-3.70 \\
3.70 \\
-7.40 \\
7.40 \\
-3.70 \\
3.70 \\
-2.96 \\
-1.48 \\
-0.74 \\
0.74 \\
1.48 \\
2.96 \\
-4.077 \\
4.07 \\
-4.81 \\
4.81 \\
-5.18 \\
5.19 \\
-5.92 \\
5.92 \\
-6.29 \\
6.29 \\
-7.03 \\
7.03 \\
-7.03 \\
7.03 \\
-6.029 \\
6.29 \\
-5.92 \\
5.92 \\
-5.18 \\
5.18 \\
-4.81 \\
4.81 \\
-4.07 \\
4.07 \\
-2.96 \\
-1.48 \\
-0.74 \\
0.74 \\
1.48 \\
2.96 \\
-2.59 \\
-1.85 \\
-0.37 \\
0.37 \\
1.85 \\
2.59 \\
-1.48 \\
-0.74 \\
0.74 \\
1.48 \\
-1.85 \\
-0.37 \\
0.37\end{array}$ & $\begin{array}{r}5.41 \\
6.41 \\
0.00 \\
0.00 \\
-6.41 \\
-6.41 \\
6.41 \\
6.41 \\
5.41 \\
6.41 \\
5.41 \\
6.41 \\
5.77 \\
5.77 \\
4.49 \\
4.49 \\
3.85 \\
3.85 \\
2.56 \\
2.56 \\
1.92 \\
1.92 \\
0.64 \\
0.64 \\
-0.64 \\
-0.64 \\
-1.92 \\
-1.92 \\
-2.56 \\
-2.56 \\
-3.85 \\
-3.85 \\
-4.49 \\
-4.49 \\
-5.77 \\
-5.77 \\
-6.41 \\
-6.41 \\
-6.41 \\
-6.41 \\
-6.41 \\
-6.41 \\
5.77 \\
5.77 \\
5.77 \\
5.77 \\
5.77 \\
5.77 \\
5.13 \\
5.13 \\
5.13 \\
5.13 \\
4.49 \\
4.49 \\
4.49\end{array}$ & $\begin{array}{l}4: 1 \\
4: 1 \\
4: 1 \\
4: 1 \\
4: 1 \\
4: 1 \\
4: 1 \\
4: 1 \\
4: 1 \\
4: 1 \\
4: 1 \\
4: 1 \\
4: 1 \\
4: 1 \\
4: 1 \\
4: 1 \\
4: 1 \\
4: 1 \\
4: 1 \\
4: 1 \\
4: 1 \\
4: 1 \\
4: 1 \\
4: 1 \\
4: 1 \\
4: 1 \\
4: 1 \\
4: 1 \\
4: 2 \\
4: 1 \\
4: 1 \\
4: 1 \\
4: 1 \\
4: 1 \\
4: 1 \\
4: 1 \\
4: 1 \\
4: 1 \\
4: 1 \\
4: 1 \\
4: 1 \\
4: 1 \\
4: 1 \\
4: 1 \\
4: 1 \\
4: 1 \\
4: 2 \\
4: 2 \\
4: 2 \\
4: 4 \\
4: 3 \\
4: 3\end{array}$ & $\begin{array}{r}5.0 \\
5.2 \\
5.8 \\
5.4 \\
5.6 \\
5.6 \\
6.8 \\
7.7 \\
7.6 \\
6.3 \\
6.3 \\
5.5 \\
5.7 \\
5.6 \\
9.0 \\
8.0 \\
10.6 \\
6.7 \\
10.6 \\
6.6 \\
9.4 \\
6.4 \\
6.7 \\
5.7 \\
7.4 \\
6.0 \\
10.0 \\
5.6 \\
11.2 \\
5.6 \\
9.6 \\
6.2 \\
9.0 \\
6.3 \\
6.3 \\
5.6 \\
5.7 \\
6.6 \\
6.8 \\
6.4 \\
6.6 \\
6.2 \\
11.0 \\
12.2 \\
11.6 \\
10.8 \\
7.6 \\
7.1 \\
12.1 \\
12.0 \\
14.6 \\
10.7 \\
16.6 \\
15.8 \\
15.5\end{array}$ & & $\begin{array}{l}1.4467 \\
1: 5334 \\
1: 7956 \\
1.6205 \\
1.7079 \\
1.7079 \\
2.2383 \\
2.6432 \\
2.5979 \\
2.0160 \\
2.0160 \\
1.6642 \\
1.7517 \\
1.7079 \\
3.2385 \\
2.7794 \\
3.9884 \\
2.1937 \\
3.9884 \\
2.1492 \\
3.4242 \\
2.0603 \\
2.1937 \\
1.7517 \\
2.5076 \\
1.8835 \\
3.7050 \\
1.7079 \\
4.2883 \\
1.7079 \\
3.5175 \\
1.9718 \\
3.2385 \\
2.0160 \\
2.0160 \\
117079 \\
1.7517 \\
2.1492 \\
2.2383 \\
2.0603 \\
211492 \\
1.9718 \\
4.1788 \\
4.7572 \\
4.4667 \\
4.0835 \\
2.5979 \\
2.3726 \\
9.7967 \\
9.7011 \\
5.9646 \\
4.0359 \\
14.3067 \\
13.4580 \\
13.1574\end{array}$ & $\begin{array}{l}G L \\
G L \\
G L \\
G L \\
G L \\
G L \\
G L \\
G L \\
G L \\
G L \\
G L \\
G L \\
G L \\
G L \\
G L \\
G L \\
G L \\
G L \\
G L \\
G L \\
G L \\
G L \\
G L \\
G L \\
G L \\
G L \\
G L \\
G L \\
G L \\
G L \\
G L \\
G L \\
G L \\
G L \\
G L \\
G L \\
G L \\
G L \\
G L \\
G L \\
G L \\
G L \\
G L \\
G L \\
G L \\
G L \\
G L \\
G L \\
S S \\
S S \\
G L \\
G L \\
S S \\
S S \\
S S\end{array}$ \\
\hline
\end{tabular}


Table B-2. (continued)

BLOCK 1-5285 (4 PSIG)

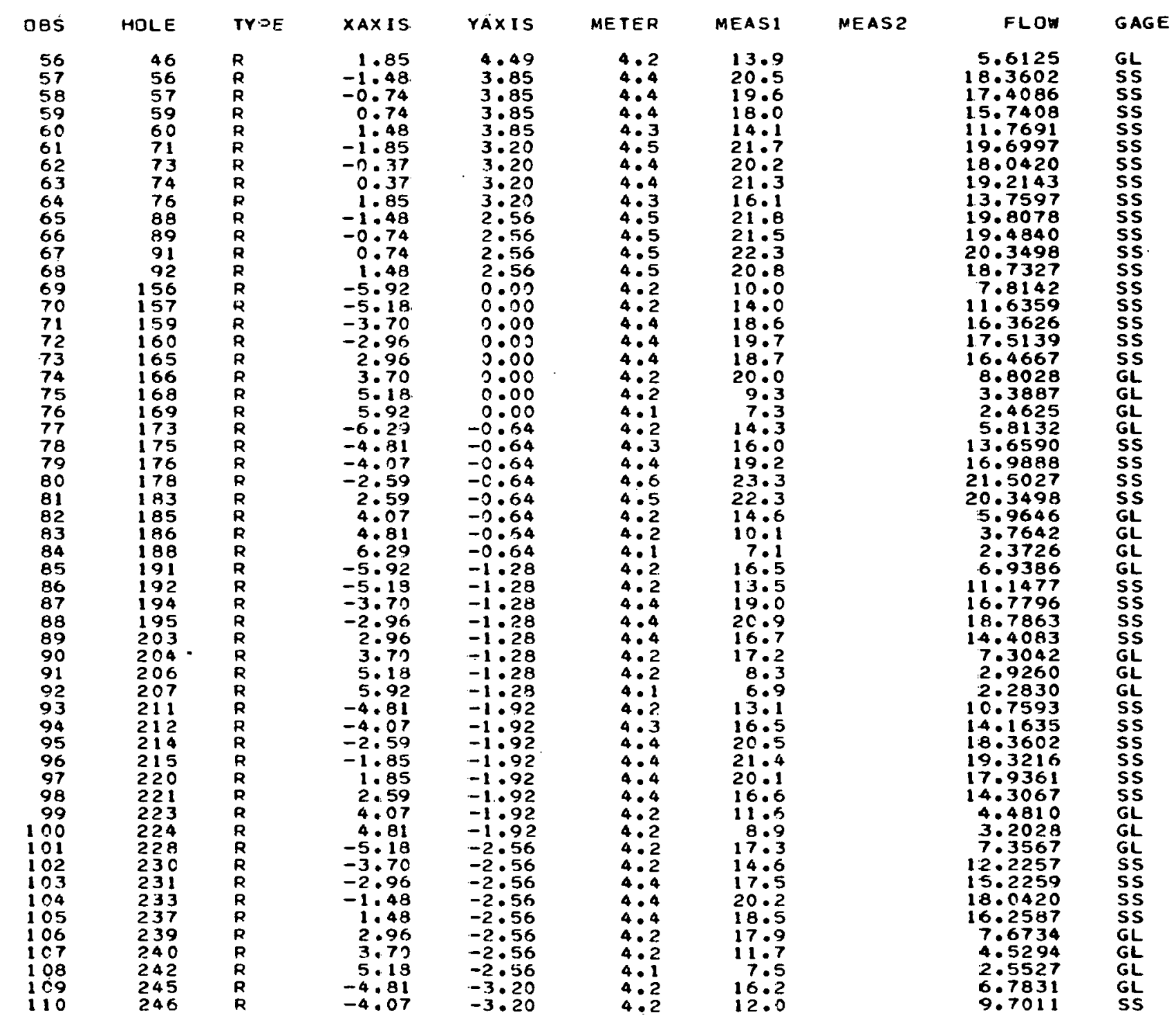


Table B-2. (continued)

BLOCK 1-5285 (4 PSIG)

\begin{tabular}{|c|c|c|c|c|c|c|c|c|c|}
\hline Des & HOLE & TYPE & XAXIS & YAXIS & ME TER & MFASI & MEAS 2 & FLOW & GAGE \\
\hline $\begin{array}{lll}1 & 1 & 1 \\
1 & 1 & 2 \\
1 & 1 & 3 \\
1 & 1 & 4 \\
1 & 1 & 5 \\
1 & 1 & 6 \\
1 & 1 & 7 \\
1 & 1 \\
1 & 1 & 9 \\
1 & 20 \\
1 & 21 \\
1 & 22 \\
1 & 2.3 \\
1 & 24 \\
1 & 25 \\
1 & 26 \\
1 & 27 \\
1 & 28 \\
1 & 29 \\
1 & 30 \\
1 & 31 \\
1 & 32 \\
1 & 33 \\
1 & 34 \\
1 & 35 \\
1 & 36 \\
1 & 37 \\
1 & 39 \\
1 & 39 \\
1 & 40 \\
1 & 41 \\
1 & 42 \\
1 & 43 \\
1 & 44 \\
1 & 45 \\
1 & 46\end{array}$ & $\begin{array}{l}248 \\
249 \\
254 \\
255 \\
257 \\
258 \\
262 \\
263 \\
271 \\
272 \\
276 \\
278 \\
285 \\
287 \\
290 \\
291 \\
299 \\
300 \\
127 \\
108 \\
124 \\
128 \\
143 \\
146 \\
179 \\
182 \\
197 \\
201 \\
217 \\
218 \\
125 \\
127 \\
162 \\
163 \\
198 \\
200\end{array}$ & $\begin{array}{l}R \\
R \\
R \\
R \\
R \\
P \\
R \\
R \\
R \\
P \\
R \\
R \\
R \\
R \\
R \\
R \\
R \\
R \\
S \\
S \\
S \\
S \\
S \\
S \\
S \\
S \\
S \\
S \\
S \\
S \\
I \\
I \\
I \\
I \\
I \\
I\end{array}$ & $\begin{array}{r}-2.59 \\
-1.35 \\
1.85 \\
2.59 \\
4.07 \\
4.91 \\
-3.70 \\
-2.96 \\
2.96 \\
3.70 \\
-4.07 \\
-2.59 \\
2.59 \\
4.07 \\
-3.70 \\
-2.96 \\
2.96 \\
3.70 \\
-0.37 \\
0: 37 \\
-1.48 \\
1.48 \\
-1.85 \\
1.85 \\
-1.85 \\
1.35 \\
-1.448 \\
1.48 \\
-0.37 \\
0.37 \\
-0.74 \\
0.74 \\
-1.48 \\
1.48 \\
-0.74 \\
0.74\end{array}$ & $\begin{array}{r}-3 \cdot 20 \\
-3.20 \\
-3.20 \\
-3.20 \\
-3.20 \\
-3.20 \\
-3.85 \\
-3.85 \\
-3.85 \\
-3.85 \\
-4.49 \\
-4.49 \\
-4.49 \\
-4.86 \\
-5.13 \\
-5.13 \\
-5.13 \\
-5.13 \\
1.92 \\
1.92 \\
1.28 \\
1.28 \\
0.64 \\
0.64 \\
-0.64 \\
-0.64 \\
-1.28 \\
-1.28 \\
-1.92 \\
-1.92 \\
1.28 \\
1.28 \\
0.00 \\
0.00 \\
-1.28 \\
-1.28\end{array}$ & $\begin{array}{l}4 \cdot ? \\
4: 4 \\
4: 3 \\
4: 2 \\
4: 2 \\
4: 2 \\
4: 2 \\
4: 2 \\
4: 2 \\
4: 2 \\
4: 2 \\
4: 2 \\
4: 2 \\
4: 1 \\
4: 1 \\
4: 2 \\
4: 2 \\
4: 1 \\
4: 4 \\
4: 4 \\
4: 5 \\
4: 44 \\
4: 5 \\
4: 4 \\
4: 5 \\
4: 5 \\
4: 5 \\
4: 5 \\
4: 5 \\
4: 5 \\
4: 4 \\
4: 4 \\
4: 4 \\
4: 4 \\
4: 4 \\
4: 4\end{array}$ & $\begin{array}{r}15.6 \\
17.8 \\
13.5 \\
17.0 \\
9.1 \\
7.6 \\
18.5 \\
11.7 \\
10.5 \\
8.5 \\
12.3 \\
10.6 \\
9.6 \\
7.5 \\
10.0 \\
11.7 \\
8.2 \\
7.2 \\
21.7 \\
22.4 \\
21.5 \\
21.2 \\
21.6 \\
21.4 \\
23.7 \\
24.0 \\
22.4 \\
22: 7 \\
22.2 \\
22.4 \\
19.8 \\
21.1 \\
20.2 \\
21.4 \\
21.1 \\
21.8\end{array}$ & & $\begin{array}{r}13.2184 \\
15.5345 \\
11.1812 \\
7.1994 \\
3.2956 \\
2.6065 \\
7.9928 \\
9.4150 \\
3.9537 \\
3.0180 \\
4.8212 \\
8.3753 \\
3.5290 \\
2.5527 \\
3.7050 \\
4.5294 \\
2.8802 \\
2.4175 \\
19.6442 \\
20.4012 \\
19.4840 \\
19.1071 \\
19.5918 \\
19.3216 \\
21.8835 \\
22.2768 \\
20.4586 \\
20.7856 \\
20.2412 \\
20.4586 \\
17.6192 \\
19.0001 \\
18.0420 \\
19.3216 \\
19.0001 \\
19.7520\end{array}$ & $\begin{array}{l}\text { SS } \\
S S \\
S S \\
G L \\
G L \\
G L \\
G L \\
S S \\
G L \\
G L \\
G L \\
S S \\
G L \\
G L \\
G L \\
G L \\
G L \\
G L \\
S S \\
S S \\
S S \\
S S \\
S S \\
S S \\
\text { SS } \\
S S \\
\text { SS } \\
\text { SS } \\
\text { SS } \\
\text { SS } \\
\text { SS } \\
\text { SS } \\
\text { SS } \\
\text { SS } \\
\text { SS } \\
\text { SS }\end{array}$ \\
\hline
\end{tabular}


Table B-2. (continued)

BLOCK : -5864 (4 PSIG)

\begin{tabular}{|c|c|c|c|c|c|c|c|c|c|}
\hline OBS & HOLE & TYPE & XAXIS & YAXIS & METER & MEASI & MEAS 2 & FLOW & GAGE \\
\hline $\begin{array}{l}1 \\
2 \\
3 \\
4 \\
5 \\
6 \\
7 \\
8 \\
9 \\
10 \\
11 \\
12 \\
13 \\
14 \\
15 \\
16 \\
17 \\
18 \\
19 \\
20 \\
21 \\
22 \\
23 \\
24 \\
25 \\
26 \\
27 \\
28 \\
29 \\
30 \\
31 \\
32 \\
33 \\
34 \\
35 \\
36 \\
37 \\
38 \\
39 \\
40 \\
41 \\
42 \\
43 \\
44 \\
45 \\
46 \\
47 \\
48 \\
49 \\
50 \\
51 \\
52 \\
53 \\
54 \\
55\end{array}$ & 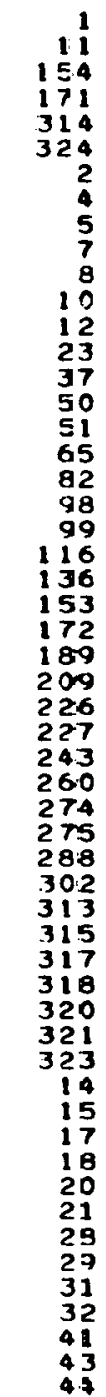 & $\begin{array}{l}C \\
C \\
C \\
C \\
C \\
C \\
F \\
F \\
F \\
F \\
F \\
F \\
F \\
F \\
F \\
F \\
F \\
F \\
F \\
F \\
F \\
F \\
F \\
F \\
F \\
F \\
F \\
F \\
F \\
F \\
F \\
F \\
F \\
F \\
F \\
F \\
F \\
F \\
F \\
F \\
F \\
F \\
R \\
R \\
R \\
R \\
P \\
R \\
R \\
R \\
R \\
R \\
R \\
R \\
R\end{array}$ & $\begin{array}{r}-3.70 \\
3.70 \\
-7.40 \\
7.40 \\
-3.70 \\
3.70 \\
-2.96 \\
-1.48 \\
-0.74 \\
0.74 \\
1.48 \\
2.96 \\
-4.07 \\
4.07 \\
-4.81 \\
4.81 \\
-5.18 \\
5.18 \\
-5.92 \\
5.92 \\
-6.29 \\
6: 29 \\
-7.03 \\
7.03 \\
-7.03 \\
7.03 \\
-6.29 \\
6.29 \\
-5.92 \\
5.92 \\
-5.18 \\
5.18 \\
-4.81 \\
4.81 \\
-4.07 \\
4.07 \\
-2.96 \\
-1.48 \\
-0.74 \\
0.74 \\
1.48 \\
2.96 \\
-2.59 \\
-1.85 \\
-0.37 \\
0.37 \\
1.85 \\
2.59 \\
-1.48 \\
-0.74 \\
0.74 \\
1.48 \\
-1.85 \\
-0.37 \\
0.37\end{array}$ & $\begin{array}{r}6.41 \\
6.41 \\
0.00 \\
0.00 \\
-6.41 \\
-6.41 \\
6.41 \\
6.41 \\
6.41 \\
6.41 \\
6.41 \\
6.41 \\
5.77 \\
5.77 \\
4.49 \\
4.49 \\
3.85 \\
3.85 \\
2.56 \\
2.56 \\
1.92 \\
1.92 \\
0.64 \\
0.64 \\
-0.64 \\
-0.64 \\
-1.92 \\
-1.92 \\
-2.56 \\
-2.56 \\
-3.85 \\
-3.85 \\
-4.49 \\
-4.49 \\
-5.77 \\
-5.77 \\
-6.41 \\
-6.41 \\
-6.41 \\
-6.41 \\
-6.41 \\
-6.41 \\
5.77 \\
5.77 \\
5.77 \\
5.77 \\
5.77 \\
5.77 \\
5.13 \\
5.13 \\
5.13 \\
5.13 \\
4.49 \\
4.49 \\
4.49\end{array}$ & 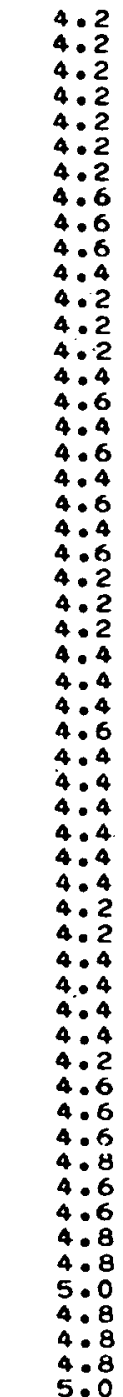 & 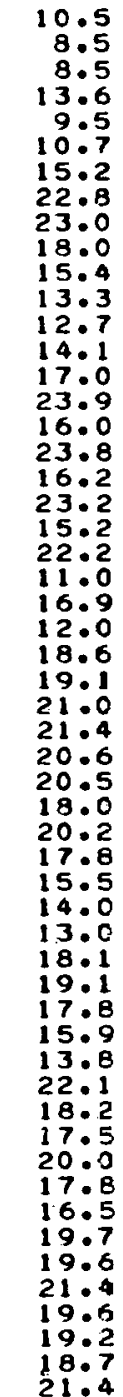 & & $\begin{array}{r}8.2814 \\
6.4306 \\
6.4306 \\
11.2451 \\
7.3500 \\
8.4692 \\
12.8199 \\
20.9533 \\
21.1727 \\
23.4702 \\
19.4674 \\
10.9533 \\
10.3728 \\
11.7339 \\
14.7140 \\
22.1659 \\
13.6992 \\
22.0551 \\
13.9012 \\
21.3926 \\
12.8961 \\
20.2980 \\
8.7517 \\
14.5267 \\
9.7011 \\
16.3626 \\
16.8841 \\
18.8931 \\
19.4310 \\
18.4666 \\
18.3602 \\
15.7408 \\
18.04420 \\
15.5345 \\
13.1963 \\
11.6359 \\
10.6625 \\
15.8441 \\
16.8841 \\
15.5345 \\
1135984 \\
11.4403 \\
20.1892 \\
23.7743 \\
22.7130 \\
26.6911 \\
23.1668 \\
21.22116 \\
26.2235 \\
26.0680 \\
29.0536 \\
26.0680 \\
25.4476 \\
24.6761 \\
29.0536\end{array}$ & $\begin{array}{l}\text { SS } \\
\text { SS } \\
\text { SS } \\
\text { SS } \\
\text { SS } \\
\text { SS } \\
\text { SS } \\
\text { SS } \\
\text { SS } \\
\text { TA } \\
\text { TA } \\
\text { SS } \\
\text { SS } \\
\text { SS } \\
\text { SS } \\
\text { SS } \\
\text { SS } \\
\text { SS } \\
\text { SS } \\
\text { SS } \\
\text { SS } \\
\text { SS } \\
\text { SS } \\
\text { SS } \\
\text { SS } \\
\text { SS } \\
\text { SS } \\
\text { SS } \\
\text { SS } \\
\text { SS } \\
\text { SS } \\
\text { SS } \\
\text { SS } \\
\text { SS } \\
\text { SS } \\
\text { SS } \\
\text { SS } \\
\text { SS } \\
\text { SS } \\
\text { SS } \\
\text { SS } \\
\text { SS } \\
\text { SS } \\
\text { TA } \\
\text { TA } \\
\text { TA } \\
\text { TA } \\
\text { TA } \\
\text { TA } \\
\text { IA } \\
\text { TA } \\
\text { TA } \\
\text { TA } \\
\text { TA } \\
\text { TA }\end{array}$ \\
\hline
\end{tabular}


Table B-2. (continued)

BLOCK 1-5864 (4 PSIG)

\begin{tabular}{|c|c|c|c|c|c|c|c|c|c|}
\hline oBs & HOLE & TYPE & XAXIS & YAXIS & METER & MEASI & MEAS 2 & FLow & GAGE \\
\hline $\begin{array}{l}56 \\
57 \\
58 \\
59 \\
60 \\
61 \\
62 \\
63 \\
64 \\
65 \\
66 \\
67 \\
68 \\
69 \\
70 \\
71 \\
72 \\
73 \\
74 \\
75 \\
76 \\
77 \\
78 \\
79 \\
80 \\
81 \\
82 \\
83 \\
84 \\
85 \\
86 \\
87 \\
88 \\
89 \\
90 \\
91 \\
92 \\
93 \\
94 \\
95 \\
96 \\
97 \\
98 \\
99 \\
100 \\
101 \\
102 \\
103 \\
104 \\
105 \\
106 \\
107 \\
108 \\
109 \\
110\end{array}$ & $\begin{array}{l}46 \\
56 \\
57 \\
59 \\
60 \\
71 \\
73 \\
74 \\
76 \\
88 \\
89 \\
91 \\
91 \\
92 \\
156 \\
157 \\
159 \\
160 \\
165 \\
166 \\
168 \\
169 \\
173 \\
175 \\
176 \\
178 \\
183 \\
185 \\
186 \\
188 \\
191 \\
192 \\
194 \\
195 \\
203 \\
204 \\
206 \\
207 \\
211 \\
212 \\
214 \\
215 \\
220 \\
221 \\
223 \\
224 \\
228 \\
230 \\
231 \\
233 \\
237 \\
239 \\
240 \\
242 \\
245 \\
246\end{array}$ & $\begin{array}{l}R \\
R \\
R \\
R \\
R \\
R \\
R \\
R \\
R \\
R \\
R \\
R \\
R \\
R \\
R \\
R \\
R \\
R \\
R \\
R \\
R \\
R \\
R \\
R \\
R \\
R \\
R \\
R \\
R \\
R \\
R \\
R \\
R \\
R \\
R \\
R \\
R \\
R \\
R \\
R \\
R \\
R \\
R \\
R \\
R \\
R \\
R \\
R \\
R \\
R \\
R \\
R \\
R \\
R \\
R \\
R \\
R \\
R \\
R \\
R \\
R \\
R\end{array}$ & $\begin{array}{r}1.85 \\
-1: 48 \\
-0: 74 \\
0.74 \\
1: 48 \\
-1.85 \\
-0.37 \\
0.37 \\
1.95 \\
-1.48 \\
-0.74 \\
0.74 \\
1.48 \\
-5.92 \\
-5.18 \\
-3.70 \\
-2.96 \\
2.96 \\
3.70 \\
5.18 \\
5.92 \\
-6.29 \\
-4.81 \\
-4.07 \\
-2.59 \\
2.59 \\
4.07 \\
4.81 \\
6.29 \\
-5.92 \\
-5.18 \\
-3.70 \\
-2.96 \\
2.96 \\
3.70 \\
5.18 \\
5.92 \\
-4.81 \\
-4.07 \\
-2.59 \\
-1.85 \\
1.85 \\
2.59 \\
4.07 \\
4.81 \\
-5.18 \\
-3.70 \\
-2.96 \\
-11.48 \\
1148 \\
2.96 \\
3.70 \\
5.18 \\
-4.81 \\
-4.07\end{array}$ & $\begin{array}{r}4.49 \\
3.85 \\
3.85 \\
3.85 \\
3.85 \\
3.20 \\
3.20 \\
3.20 \\
3.20 \\
2.56 \\
2.56 \\
2.56 \\
2.56 \\
0.00 \\
0.00 \\
0.00 \\
0.00 \\
2.00 \\
0.00 \\
0.00 \\
0.00 \\
-0.64 \\
-0.64 \\
-0.64 \\
-0.64 \\
-0.64 \\
-0.64 \\
-0.64 \\
-0.64 \\
-1.28 \\
-1.28 \\
-1.28 \\
-1.28 \\
-1.28 \\
-1.28 \\
-1.28 \\
-1.28 \\
-1.92 \\
-1.922 \\
-1.922 \\
-1.92 \\
-1.92 \\
-1.92 \\
-1.92 \\
-1.92 \\
-2.56 \\
-2.56 \\
-2.56 \\
-2.56 \\
-2.56 \\
-2.56 \\
-2.56 \\
-2.56 \\
-3.20 \\
-3.20\end{array}$ & 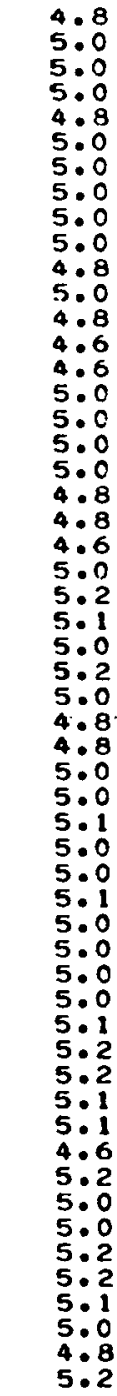 & 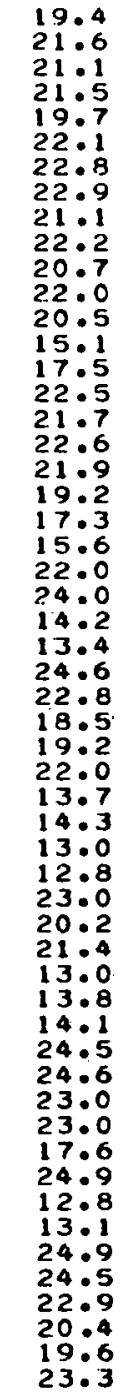 & $\begin{array}{l}16.6 \\
15.5 \\
16.0 \\
16.6 \\
15.1 \\
14.7 \\
15.0 \\
16.1 \\
16.5\end{array}$ & 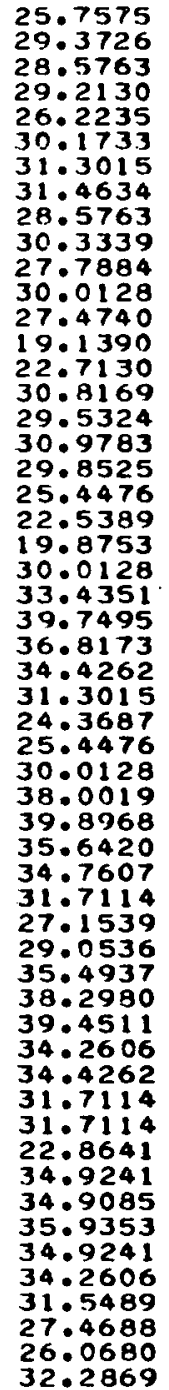 & 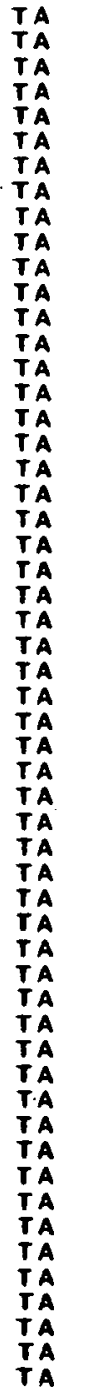 \\
\hline
\end{tabular}


Table B-2. (continued)

3LOCK 1-5864 (4 PSIG)

\begin{tabular}{|c|c|c|c|c|c|c|c|c|c|}
\hline oes & HOLE & TYPE & $\times 4 \times 15$ & YAXIS & METER & MËAS 1 & MEAS 2 & FLOW & GAGE \\
\hline 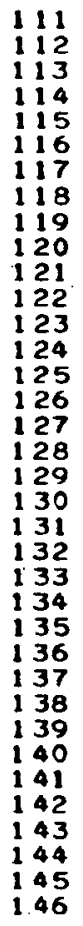 & $\begin{array}{l}248 \\
249 \\
254 \\
255 \\
257 \\
258 \\
262 \\
263 \\
271 \\
272 \\
276 \\
278 \\
285 \\
287 \\
290 \\
291 \\
299 \\
300 \\
107 \\
108 \\
124 \\
123 \\
143 \\
146 \\
179 \\
182 \\
197 \\
201 \\
217 \\
218 \\
125 \\
127 \\
162 \\
163 \\
198 \\
200\end{array}$ & $\begin{array}{l}R \\
R \\
R \\
R \\
R \\
R \\
R \\
R \\
R \\
R \\
R \\
R \\
R \\
R \\
R \\
R \\
R \\
R \\
R \\
R \\
R \\
S \\
S \\
S \\
S \\
S \\
S \\
S \\
S \\
S \\
S \\
S \\
S \\
S \\
I \\
I \\
I \\
1 \\
I \\
I\end{array}$ & $\begin{array}{r}-2.59 \\
-1.85 \\
1.85 \\
2.59 \\
4.07 \\
4.81 \\
-3.70 \\
-2.96 \\
2.96 \\
3.70 \\
-4.07 \\
-2.59 \\
2.59 \\
4.07 \\
-3.70 \\
-2.96 \\
2.96 \\
3.70 \\
-0.37 \\
0.37 \\
-1.48 \\
1.48 \\
-1.85 \\
1.35 \\
-1.95 \\
1.35 \\
-1.48 \\
1.48 \\
-0.37 \\
0.37 \\
-0.74 \\
0.74 \\
-1.48 \\
1.48 \\
-0.74 \\
0.74\end{array}$ & $\begin{array}{r}-3.20 \\
-3.20 \\
-3.20 \\
-3.20 \\
-3.20 \\
-3.20 \\
-3.85 \\
-3.85 \\
-3.85 \\
-3.85 \\
-4.49 \\
-4.49 \\
-4.49 \\
-4.86 \\
-5.13 \\
-5.13 \\
-5.13 \\
-5.13 \\
1.92 \\
1.92 \\
1.28 \\
1.28 \\
0.64 \\
0.64 \\
-0.64 \\
-0.64 \\
-1.28 \\
-1.28 \\
-1.92 \\
-1.92 \\
1.29 \\
1.28 \\
0.00 \\
0.00 \\
-1.28 \\
-1.28\end{array}$ & $\begin{array}{l}5.0 \\
5.2 \\
5: 1 \\
5: 2 \\
5.0 \\
4: 8 \\
5.0 \\
5.0 \\
4.8 \\
4.8 \\
5.0 \\
5.0 \\
5.0 \\
4.6 \\
4.6 \\
4.8 \\
4.6 \\
4.4 \\
5: 0 \\
5.0 \\
4.8 \\
5.0 \\
4.8 \\
4.8 \\
5.0 \\
5.0 \\
5.0 \\
5.2 \\
5.0 \\
4.8 \\
4.8 \\
4.8 \\
4.8 \\
4.8 \\
4.8 \\
5.0\end{array}$ & 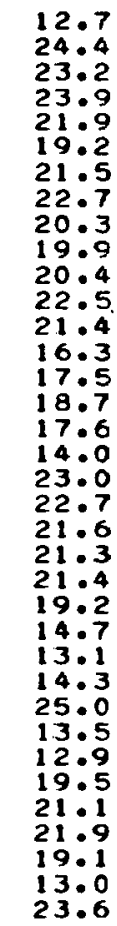 & $\begin{array}{l}17.2 \\
15.2 \\
16.6 \\
15.5 \\
14.8\end{array}$ & $\begin{array}{l}34.4688 \\
34.0951 \\
32.0368 \\
33.0706 \\
29.8525 \\
25.4476 \\
29.2130 \\
31.1398 \\
27.1603 \\
26.5351 \\
27.4688 \\
30.8169 \\
29.0536 \\
20.9135 \\
22.7130 \\
24.6761 \\
22.8641 \\
17.4324 \\
31.6254 \\
31.11398 \\
29.2116 \\
28.8943 \\
28.8941 \\
25.4476 \\
41.2821 \\
35.9953 \\
39.7836 \\
35.09904 \\
36.9628 \\
34.8515 \\
25.99126 \\
28.4192 \\
29.6891 \\
25.2930 \\
35.2899 \\
32.6013\end{array}$ & $\begin{array}{l}\text { TA } \\
\text { TA } \\
\text { TA } \\
\text { TA } \\
\text { TA } \\
\text { TA } \\
\text { TA } \\
\text { TA } \\
\text { TA } \\
\text { TA } \\
\text { TA } \\
\text { TA } \\
\text { TA } \\
\text { TA } \\
\text { TA } \\
\text { TA } \\
\text { TA } \\
\text { TA } \\
\text { IA } \\
\text { TA } \\
\text { TA } \\
\text { TA } \\
\text { TA } \\
\text { TA } \\
\text { TA } \\
\text { TA } \\
\text { TA } \\
\text { TA } \\
\text { TA } \\
\text { TA } \\
\text { TA } \\
\text { TA } \\
\text { TA }\end{array}$ \\
\hline
\end{tabular}




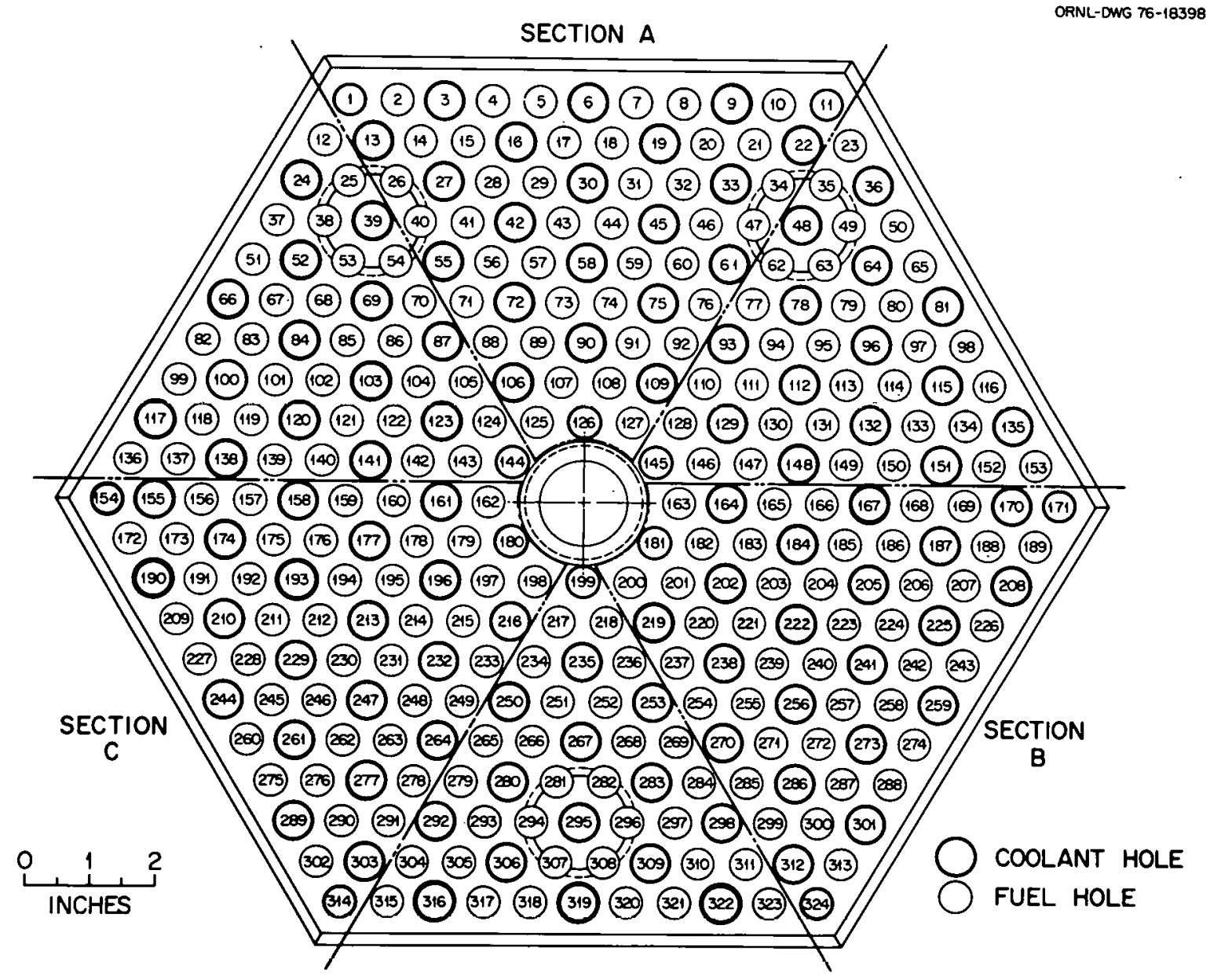

Fig. B-1. Top view of HTGR graphite Fuel-element block. 


\section{THIS PAGE \\ WAS INTENTIONALLY \\ LEFT BLANK}


1-2. Central Research Library

3. Document Reference Section

4-5. Laboratory Records Dept.

6. Laboratory Records, R.C.

7. ORNL Patent Office

8. P. Angelini

9-11. B. J. Baxter

12-16. C. K. Bayne

17. R. J. Beaver

18. M. Bender

19. R. Blumberg

20. R. J. Braatz

21. R. A. Bradley

22-25. A. J. Caputo

26. J. A. Carpenter

27. W. L. Carter

28. H. E. Cochran

29: J. H. Coobs

30. D. A. Costanzo

31. F. C. Davis

32. J. P. Drago

33. R. G. Donnelly

34. B. C. Duggins

35. W. P. Eatherly

36. I I. Federer

37. D. E. Ferguson

38. C. L. Fitzgerald

39. R. W. Glass

40. P. A. Haas

41. C. C. Haws

42. W. R. Hamel

13. F. E. Harrington

44. J. L. Heck

45. L. C. Hensley

46-48. M. R. Hill

49. R. M. Hill

50. F. J. Homan

51-55. D. R. Johnson

56. M. J. Kania
- 57-58. P. R. Kasten

59. W. J. Lackey

60. G. M. Lawson

61. K. H. Lin

62. T. B. Lindemer

63. E. L. Long, Jr.

64-65. A. L. Lotts

66. J. E. Mack

67. A.P. Malinauskas

68. S. R. McNeany

69. D. L. Million

70. K. J. Notz

71. A. R. Olsen

72. M. K. Preston

73. R. H. Rainey

74. D. P. Reid

75. A. D. Ryon

76. J. E. Rushton

77. T. F. Scanlan

78. C. D. Scott

79. J. H. Shaffer

80. J. W. Snider

81. P. E. Stein

82. D. P. Stinton

83. R. R. Suchomel

84. V. J. Tennery

85. S. M. Ticgs

86. T. N. Teigs

87. D. B. Trauger

88. V.C. A. Vaughen

89. J. R. Weir

90. R. G. Wymer

91. R. M. Young

92. R. W. Balluffi (consultant)

93. P. M. Brister (consultant)

94. W. R. Hibbard, Jr. (consultant)

95. Hayne Palmour III (consultant)

96. N. E. Promisel (consultant)

97. D. T. Stein (consultant) 
EXTERNAL DISTRIBUTION

98-105. ERDA DIVISION OF NUCLEAR FUEL CYCLE AND PRODUCTION, Washington: DC 20545

Director (2)

R. G. Bradley (1)

W. S. Schieb (5)

106-107. ERDA DIVISION OF REACTOR NUCLEAR RESEARCH AND APPLICATIONS, Washington, DC 20545

Director

108-109. ERDA IDAHO OPERATIONS OFFICE, P.O. Box 2108, Idaho Falls, ID 83401

C. E. Williams, Manager

Barry Smith

110. ERDA OFFICE OF PROGRAM MANAGEMENT, RESEARCH AND SPACE

PROGRAMS, P.O. Box 81325, San Diego, C.A 92138

J. B. Radcliffc

111. ERDA SAN FRANCISCO OPERATIONS OFFICE, 1333 Broadway, Wells Fargo Bldg., Oakland, CA 94612

R. D. Thorne, Manager

112-114. ERDA OAK RIDGE OPERATIONS OFFICE, P.O. Box E, Oak Ridge, TN 37830

Director, Research and Technical Support Division

Director, Reactor Division

F. E. Dearing, Reactor Division

115-291: ERDA TECHNICAL INFORMATION CENTER, P.O. Box 62, Oak Ridge, TN 37830

For distribution as shown in TID-4500 Distribution Category,

UC-77 - Gas-Cooled Reactor Technology 